\title{
Recommended Practice for Use of Faraday Probes in Electric Propulsion Testing
}

\author{
Daniel L. Brown* \\ U.S. Air Force Research Laboratory, Edwards Air Force Base, California 93524 \\ Mitchell L. R. Walker \\ Georgia Institute of Technology, Atlanta, Georgia 30332 \\ James Szabo \\ Busek Co., Inc., Natick, Massachusetts 01760 \\ Wensheng Huang $\underline{\S}$ \\ NASA John H. Glenn Research Center, Cleveland, Ohio 44135 \\ and \\ John E. FosterI \\ University of Michigan, Ann Arbor, Michigan 48109 \\ DOI: $10.2514 / 1 . B 35696$
}

\begin{abstract}
Faraday probes are a common plasma diagnostic used to determine the local ion charge flux of electric propulsion plumes. Standard practices, guidelines, and recommendations are provided for experimental methods and analysis techniques that aim to standardize community practices, to mitigate test environment effects, and to reduce systematic measurement error in order to improve plume predictions in the space environment. The approaches are applicable to time-averaged plasma properties in the near-field and far-field of electric propulsion plumes, with emphasis on Hall effect thrusters and gridded ion thrusters. Considerations for other electric propulsion technologies are provided, including electrosprays, arcjets, and electromagnetic thruster concepts. These test strategies are expected to increase the quality of comparisons between different thrusters and vacuum environments, thereby broadening the applicability of ground-based measurements and enhancing the fidelity for on-orbit predictions and modeling validation.
\end{abstract}

\section{Nomenclature}

$A_{C} \quad=$ cross-sectional geometric area, $\mathrm{m}^{2}$

$A_{G} \quad=$ Richardson constant, $\mathrm{A} \cdot \mathrm{cm}^{-2} \cdot \mathrm{K}^{-2}$

$C_{1,2}=$ curve-fit parameters

$D_{T} \quad=$ thruster diameter, $\mathrm{m}$

$E_{\delta} \quad=\quad$ error metric of divergence angle

$E_{\theta} \quad=\quad$ error metric of travel angle

$e=$ elementary charge; $1.6022 \times 10^{-19} \mathrm{C}$

$F_{t} \quad=$ thrust loss parameter

$h_{C} \quad=$ height of collector, $\mathrm{m}$

$h_{\mathrm{GR}}=$ height of guard ring, $\mathrm{m}$

$I_{\text {Axial }}=$ axial component of ion beam current parallel to thrust axis, A

$I_{\text {Beam }}=$ integrated ion beam current, $\mathrm{A}$

$I_{d}=$ thruster discharge current, $\mathrm{A}$

$I_{\mathrm{FP}} \quad=$ measured ion current on the Faraday probe collector, $\mathrm{A}$

$j=$ ion current density, $\mathrm{A} \cdot \mathrm{m}^{-2}$

$J_{\text {Bohm }}=$ Bohm ion current density, $\mathrm{A} \cdot \mathrm{m}^{-2}$ $j_{t} \quad=$

k $\quad=$

thermionic emission ion current density, $\mathrm{A} \cdot \mathrm{m}^{-2}$

propellant charge state index; $0,1,2,3$, etc., for $\mathrm{Xe}^{0}$, $\mathrm{Xe}^{+1}, \mathrm{Xe}^{+2}, \mathrm{Xe}^{+3}$

Received 15 December 2014; revision received 1 July 2016; accepted for publication 5 July 2016; published online 14 September 2016. This material is declared a work of the U.S. Government and is not subject to copyright protection in the United States. Copies of this paper may be made for personal and internal use, on condition that the copier pay the per-copy fee to the Copyright Clearance Center (CCC). All requests for copying and permission to reprint should be submitted to CCC at www.copyright.com; employ the ISSN 0748-4658 (print) or 1533-3876 (online) to initiate your request.

*Chief, Liquid Rocket Engines Branch, Aerospace Systems Directorate. Associate Fellow AIAA.

'Associate Professor, High-Power Electric Propulsion Laboratory. Associate Fellow AIAA.

${ }^{\ddagger}$ Chief Scientist for Hall Thrusters. Associate Fellow AIAA.

${ }^{\S}$ Research Engineer, Propulsion and Propellants. Member AIAA.

"Associate Professor, Nuclear Engineering and Radiological Sciences. Associate Fellow AIAA.

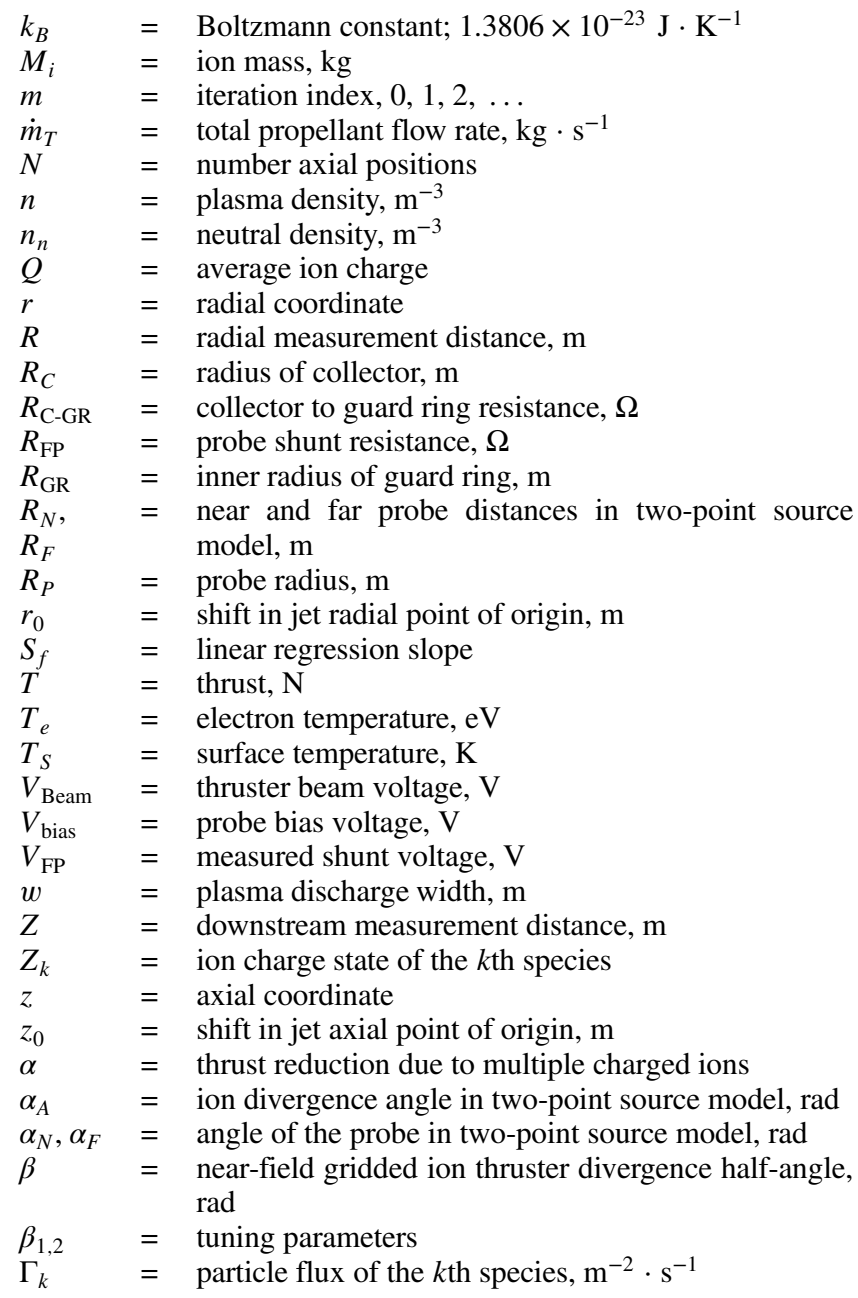


$\gamma_{k}=$ secondary electron emission yield of the $k$ th species, electrons/ion

$\delta=$ near-field Hall effect thruster divergence half-angle, $\mathrm{rad}$

$\delta_{j} \quad=$ jet divergence angle, $\mathrm{rad}$

$\varepsilon=$ surface emissivity

$\varepsilon_{o} \quad=$ permittivity of free space; $8.8542 \times 10^{-12} \mathrm{~F} \cdot \mathrm{m}^{-1}$

$\eta_{\text {Beam }}=$ beam divergence utilization

$\eta_{\text {Current }}=$ current utilization

$\eta_{\text {Mass }}=$ mass utilization

$\theta=$ angular coordinate

$\theta_{j} \quad=$ jet travel angle, rad

$\kappa_{A}=$ correction for ion angle of incidence

$\kappa_{D}=$ correction for probe distance to thruster

$\kappa_{G} \quad=$ correction for probe ion collection area

$\kappa_{\mathrm{SEE}}=$ correction for collector secondary electron emission

$\lambda=$ far-field divergence half-angle, $\mathrm{rad}$

$\lambda_{D} \quad=\quad$ Debye length, $\mathrm{m}$

$\lambda_{\text {MFP }}=$ mean free path, $\mathrm{m}$

$\sigma \quad=$ collision cross section, $\mathrm{m}^{2}$

$\sigma_{\mathrm{SB}}=$ Stefan-Boltzmann constant; $5.6704 \times 10^{-8} \mathrm{~W} \cdot \mathrm{m}^{-2} \cdot \mathrm{K}^{-4}$

$\Phi=$ azimuthal angle, $\mathrm{rad}$

$\varphi=$ work function, $\mathrm{eV}$

$\Psi \quad=$ probe angle, $\mathrm{rad}$

$\Omega_{k} \quad=$ ion current fraction of the $k$ th species

\langle\rangle$_{J} \quad=$ charge flux-weighted average quantity

\section{Introduction}

$\mathbf{F}$ ARADAY probe measurements of ion charge flux in the plume of spacecraft electric propulsion (EP) technologies are used for several purposes, including 1) ground predictions and flight measurements of local plume properties to determine plasma-spacecraft interactions, 2) characterization of global plume properties to assess the thruster plasma discharge and loss mechanisms, 3) validation data for modeling and simulation, and 4) data for thruster acceptance tests. In addition to $\mathrm{EP}$, these diagnostics have been used for many applications, such as space plasma experiments, dense plasma focus experiments, and laserproduced plasma [1-4]. In its simplest form, the Faraday probe consists of a collector electrode that measures local ion charge flux. Other configurations use collimators, guard rings, or biased grids to filter charged particles, such as the Faraday cup. Although Faraday probe design and implementation is straightforward, the test methodology and data analysis required for $\mathrm{EP}$ technologies are complicated by systematic measurement errors.

This paper provides recommendations for time-averaged Faraday probe measurements in the near-field and far-field plumes of Hall effect thrusters (HETs) and gridded ion thrusters (GITs) to provide common test methodologies, diagnostic design, and analysis techniques. Although this paper is applicable to other EP technologies, there is insufficient data in the literature to establish standard methods for electrosprays, arcjets, and electromagnetic thruster concepts. Section II discusses the applicability to EP technologies and considerations of the plasma environment. Sections III and IV describe experimental apparatus and test methodologies, respectively. Section $\underline{V}$ presents data reduction and analysis techniques. Section VI provides information on measurement error and uncertainty. Section VII discusses probe design considerations. Section VIII contains considerations for other EP technologies. A tabulated summary of all guidelines and recommendations is provided in Appendix A, and an analysis method to remove the cathode plume is included in Appendix B.

The guidance in this paper uses precise definitions for the words should and may. The word should denotes the statement is advisory; there may be circumstances when the statement is ignored, but the associated implications must be understood and accounted for. The word may denotes the statement is a recommendation, and it is considered discretionary.

\section{A. Electric Propulsion Technologies}

II. Applicability

In EP systems, electrical energy is added to the propellant from an external power source to ionize and accelerate propellant to high exhaust velocities; this is in contrast to chemical rockets where the propellant exhaust velocity is limited to less than $5 \mathrm{~km} / \mathrm{s}$ by the energy released during propellant combustion processes. Thus, EP technologies decouple the available energy from the propellant chemical reactions, and they impart energy through electric heating, electrostatic fields, and/or electromagnetic fields. Additional details on EP thruster concepts and plasma processes are found in the literature $[\underline{5}, \underline{6}]$.

This paper focuses on HETs and GITs due to the flight heritage and extensive Faraday probe measurements in ground testing, which enables development of guidelines and recommendations. Examples of Faraday probe measurements and analysis of HET and GIT plumes are provided, with particular consideration for HET plumes due to the more recent investigations that have further improved test methodologies and analyses to reduce systematic measurement uncertainty. In addition, HETs may have additional complexities associated with annular geometry, oscillatory behavior, and facility effects on the plasma ionization and acceleration processes. The guidance for HETs and GITs may also serve as a starting point for Faraday probe measurements of other EP technologies, as discussed in Sec. VIII.

In HETs, propellant ionization and acceleration are achieved with orthogonal electric and magnetic fields in the annular discharge channel, as shown in Fig. 1 for a typical stationary plasma thruster configuration. The guidelines and recommendations in this paper are based primarily on numerous past experiments with stationary plasma thrusters; however, the paper is applicable to alternative HET configurations, including nested channel HETs and the thruster with anode layer variants [7-9]. In HETs, a constant potential difference is applied between the anode and cathode, where the cathode may be located external to the thruster body (as in Fig. 1) or centrally mounted on the thruster centerline axis. The cathode provides electrons to the plasma discharge and to neutralize the ion beam. The anode often serves as the propellant gas distributor. The HET electric and magnetic fields are designed such that electrons are confined in the plasma discharge and have a net azimuthal motion known as the Hall effect, which is the origin of the device name. Said electrons ionize propellant through a cascade of electron impact collisions. The generated ions are weakly magnetized and are accelerated by the axial electric field to generate thrust. Plasma in the HET channel is

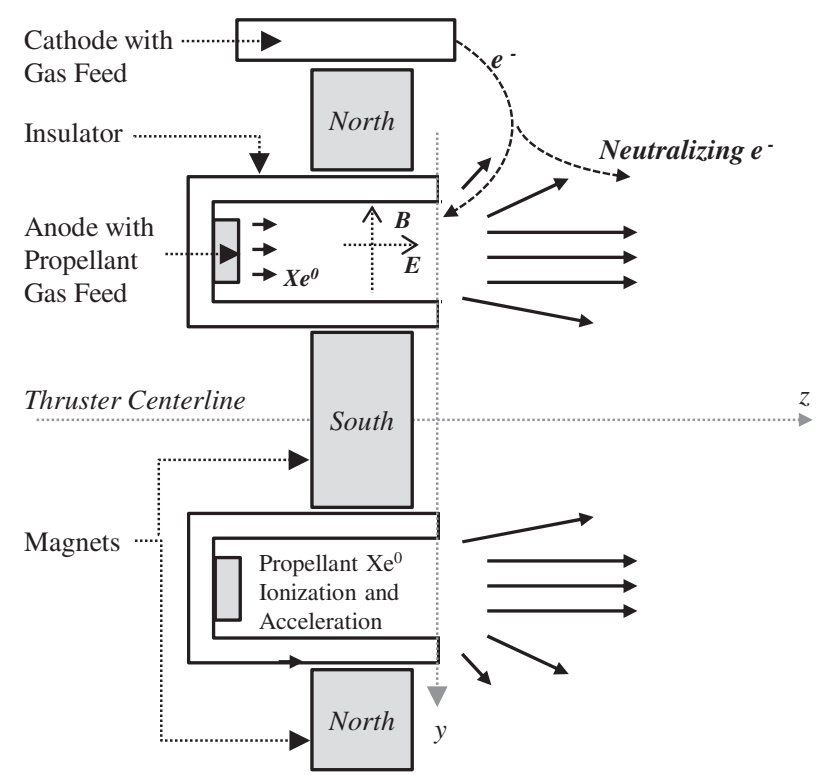

Fig. 1 Schematic of design features and plasma properties for HETs. 
quasi neutral, and thus the extracted current density is not spacecharge limited.

The GIT plasma is generated within an enclosed discharge chamber via a direct current (dc) discharge, a radio-frequency (RF) discharge, or a microwave discharge [6]. Neutral propellant gas is injected into the discharge chamber and ionized by electrons from the discharge cathode, as shown in Fig. 2, or by alternating electric and magnetic field in the case of RF or microwave discharge chambers. Magnetic fields are typically used near the anode wall to confine energetic electrons, and thereby improve ionization processes. Propellant ions are extracted, focused, and accelerated to generate thrust by the ion optics, which are typically a series of closely spaced, multiaperture grids terminating at the thruster exit plane. The ion optics geometry determines ion trajectories, particle grid interactions, and beam divergence. Maximum ion current density extracted though the ion optics is limited by space-charge considerations; thus, the maximum GIT ion beam current density is lower than a HET beam. The exhaust ion plume is neutralized by an externally mounted hollow cathode.

A Faraday probe is typically employed to assess time-averaged ion current density in the plume. Time-resolved Faraday probe measurements at tens of kilohertz are feasible (up to low megahertz are possible) with existing capabilities, as demonstrated by local plasma measurements with ion saturation reference probes at fixed bias voltages that were used in experiments with high-speed dual Langmuir probes in the near-field of HETs [10,11]. However, timeresolved Faraday probes have not been used to map an EP plume or to evaluate facility effects. Although certain guidelines related to timeaveraged Faraday probe measurements may be applicable to timeresolved measurements, there may be significant differences in the probe configuration, data acquisition, data analysis, measurement error, and quantification of ion beam parameters. These differences are beyond the scope of this paper.

The guidelines in this paper were generated for inert, nonreactive propellants such as the noble gases. Faraday probes may also be used with low vapor pressure ("condensable") and reactive propellants, such as bismuth, iodine, mercury, and ionic liquids [12,13]. Although this paper is applicable to HETs and GITs with these propellants, additional issues may arise that should be accounted for. For example, a possible concern with condensable propellants is the potential for contamination of dielectric surfaces leading to electrical leakage from the collector. Laboratory HET measurements with iodine propellant showed negligible accumulation on test coupons in the plume [14]. Mercury GITs on the Space Electric Rocket Test 2

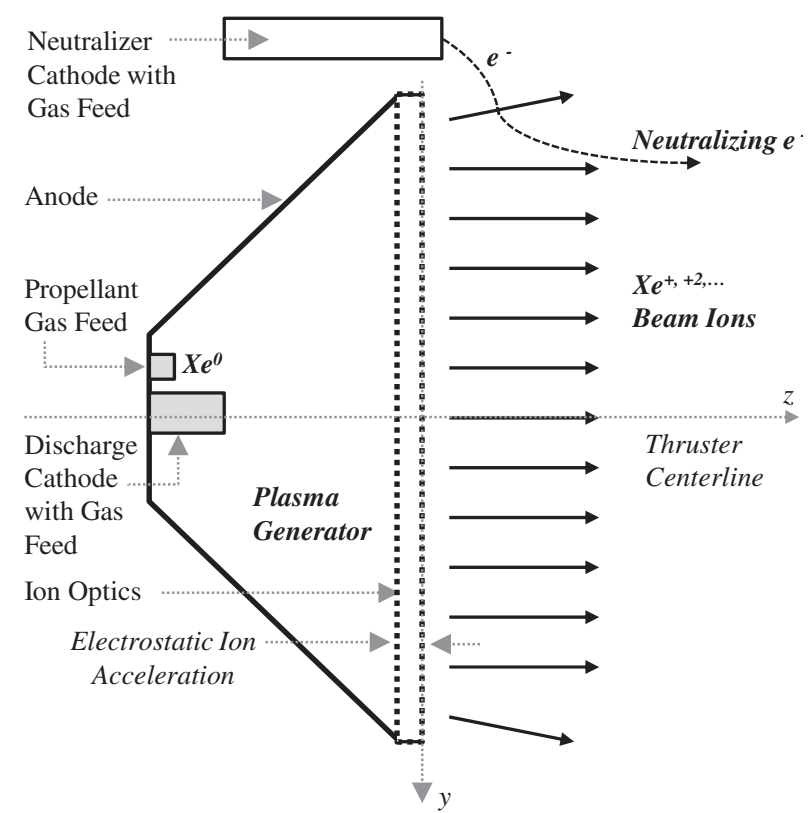

Fig. 2 Schematic of design features and plasma properties for GITs.
(SERT II) spacecraft were successfully fired on orbit for $4000 \mathrm{~h}$, where most critical spacecraft surfaces, such as solar arrays, were too warm to permit mercury condensation and no evidence of condensate accumulation was expected [15]. However, the issues unique to condensable and reactive propellant should be carefully considered for each application, such as deposition of propellant upon probe electrodes, deposition on ceramic surfaces leading to reduced electrical isolation, leakage current, and material degradation due to corrosion.

\section{B. Plasma Plume Characterization}

The plume of EP thrusters is associated with dynamic and complex processes that must be considered for Faraday probe measurements. Examples include downstream regions of ion acceleration, spatial and temporal gradients in plasma properties, and interactions with neutrals. In addition, there are thruster specific processes such as beamlet interactions downstream of GIT ion optics or HET ion beam merging from opposing sides of the annular channel. These challenges will be addressed with regard to experimental configuration, test methods, and analysis in Secs. III, IV, and V, respectively.

Facility effects influence thruster operation and the plasma plume, and they are well characterized in the EP literature [16-21]. These interactions are present in both the near-field and far-field regions, including the influence of facility background neutrals, backsputtering of facility surfaces, pressure gradients due to facility configuration and pumping, and the thruster discharge circuit in a grounded vacuum test facility. The interactions are inherent in ground-based EP plume investigations, and thus cannot be completely eliminated to fully replicate the space environment. The two primary facility interactions on the thruster plume are related to facility background neutrals, and they are illustrated in Figs. 3 and $\underline{4}$ for HETs and GITs, respectively. The first interaction is collisional processes between facility neutrals and the thruster beam, where ion-neutral charge exchange (CEX) collisions are the dominant interaction. The second interaction is ingestion, ionization, and acceleration of facility neutrals.

The influence of collisional processes between facility neutrals and the thruster beam may be evaluated with respect to the neutral particle populations in EP plumes, as shown in Fig. 5 for simulations of the BPT-4000 HET [22]. In Fig. 5, the ambient facility neutral density exceeds the thruster neutral density beyond approximately $0.02 \mathrm{~m}$, which is comparable to GIT plumes for a similar propellant flow rate [23]. The mean free path (MFP) in Eq. (1) represents the mean distance a fast particle will travel in a background of stationary neutrals before a collision:

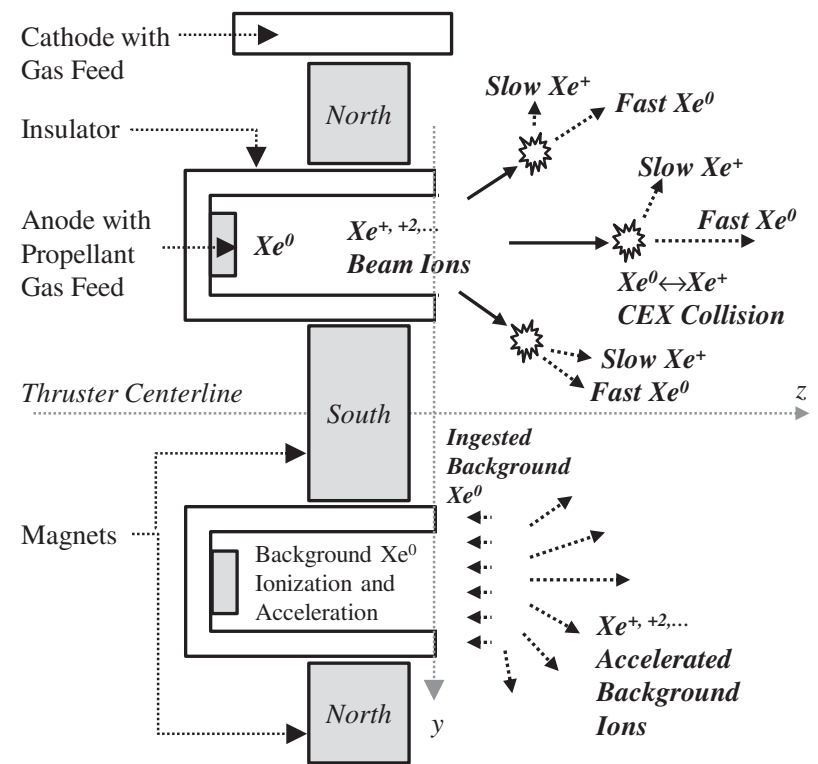

Fig. 3 Diagram of HET plasma interactions of facility background neutrals. 


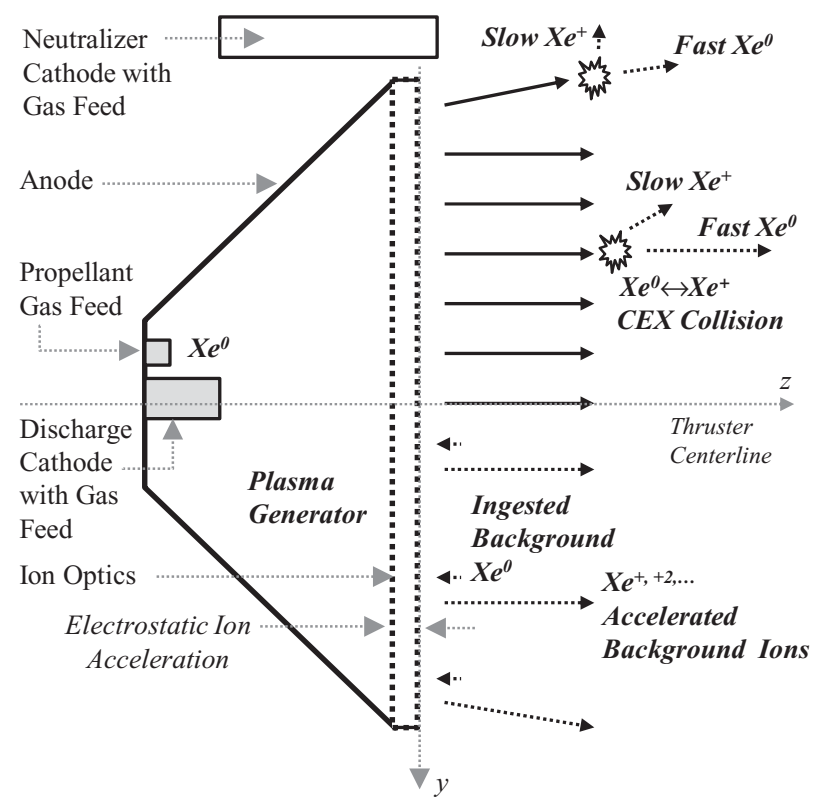

Fig. 4 Diagram of GIT plasma interactions of facility background neutrals.

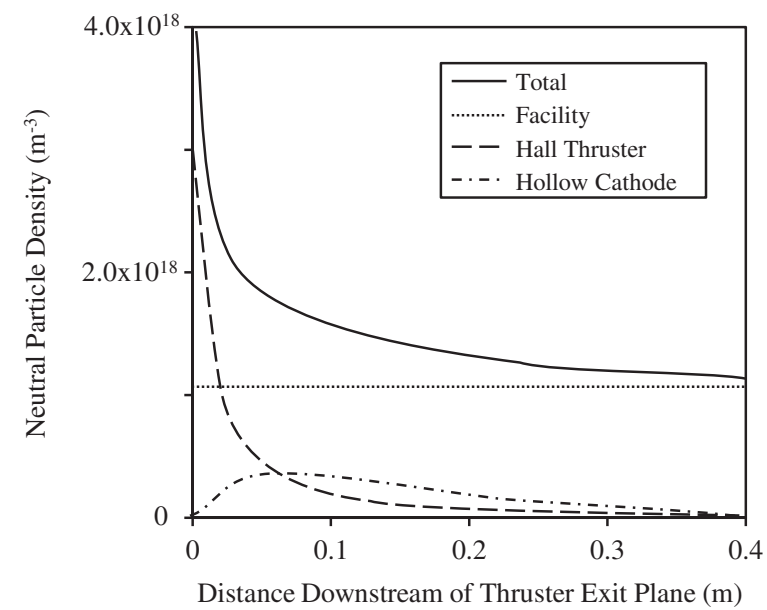

Fig. 5 Neutral particle density on thruster centerline, recreated from [22].

$$
\lambda_{\mathrm{MFP}}=\left(n_{n} \sigma\right)^{-1}
$$

In Eq. (1), $n_{n}$ is the neutral density and $\sigma$ is the particle collision cross section. The MFP may be used to determine the dominant beam collisional processes in the plume region; an overview of particle collisions and relevant cross sections was described elsewhere $[6,24,25]$. The cross section for CEX collisions between ambient xenon neutrals and xenon ions has been experimentally measured as $5 \times 10^{-19}$ to $8 \times 10^{-19} \mathrm{~m}^{2}$ [25], which was much larger than the cross section for xenon-neutral elastic scattering $\left(10^{-20} \mathrm{~m}^{2}\right)$, as well as xenon ionization and excitation cross sections $\left(10^{-20} \mathrm{~m}^{2}\right)[6,26]$. The CEX collisions between background neutral particles and beam ions generated high-velocity neutrals and low-energy scattered ions. The CEX collisional processes scattered beam ions and increased ion current density on the periphery of the plume, thereby increasing thruster plume divergence $[18,27,28]$. Based on Eq. (1), the xenon ion-neutral MFP of CEX collisions is shown in Fig. 6. For a HET or GIT operating at a $300 \mathrm{~V}$ discharge condition, the relative velocity between the xenon ions and neutral atoms was approximately $17,000 \mathrm{~m} / \mathrm{s}$ and the CEX cross section was $6 \times 10^{-19} \mathrm{~m}^{2}$ [25]. For a background neutral pressure of $1.3 \times 10^{-5}$ torr of xenon gas (torr-Xe), the neutral number density was $4.2 \times 10^{17} \mathrm{~m}^{-3}[29]$ and

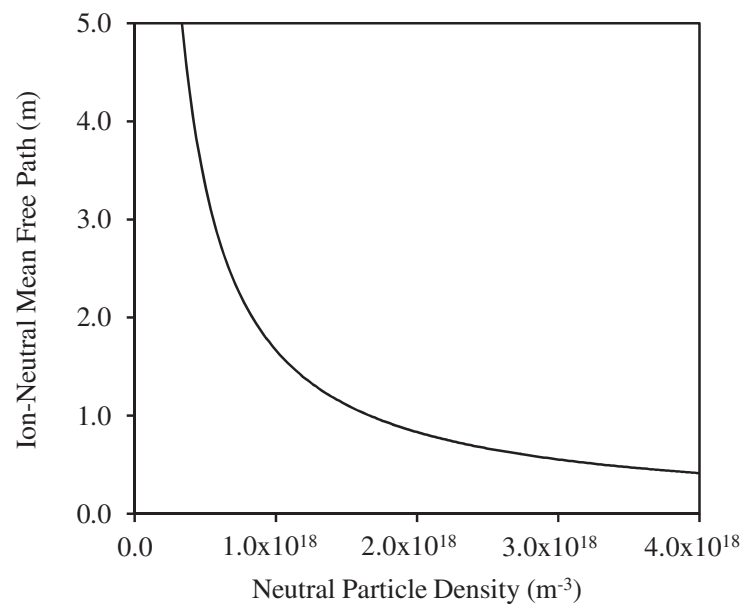

Fig. 6 Xenon ion-neutral CEX mean free path for expected neutral particle density in the near-field plume, for $\sigma=6 \times 10^{-19} \mathrm{~m}^{2}$.

the ion-neutral CEX collision MFP was $3.97 \mathrm{~m}$. The MFP values in Fig. 6 are on the order of the far-field spatial measurement region, and they are consistent with far-field experimental results showing increased plume divergence at elevated facility pressure in Sec. V. Although the ion-neutral CEX MFP is larger than the near-field spatial region of interest for most HETs and GITs, the difference is not significant; thus, CEX collisions are expected to have a nonnegligible effect in the near-field plume. These observations will be discussed with experimental results in Sec. V.

The second dominant facility interaction is associated with ingestion of facility background neutrals near the thruster discharge exit, which increases the amount of propellant used by the thruster. These ingested neutrals undergo ionization and subsequent acceleration, which increases the amount of propellant available to the thruster. The ingested facility neutrals may be ionized and accelerated downstream of the peak electric field, and thus manifest as a low-velocity divergent ion population in the beam. In GITs, the accelerator grid current may become prohibitively high at an elevated background pressure due to a CEX ion current, and therefore operation at these elevated pressures is often impractical well before ingestion effects become a significant factor. This is further discussed in Sec. IV.E.

Multiple studies have evaluated Faraday probe design modifications to minimize facility effects associated with probe collection of lowenergy CEX ions and ions generated from ingested propellant, including probe filtering mechanisms and collimators [1,30-32]. Although these techniques successfully mitigate the collection of lowenergy ions generated through facility interactions, they introduce additional error that is not representative of thruster plumes in the space environment. The impact of these facility interactions on Faraday probe measurements will be discussed, and guidelines to mitigate and account for the effects will be recommended.

In theory, computational simulations of the EP plume allow isolation and elimination of facility pressure effects to predict the on-orbit ion beam. However, this predictive capability relies on accurate plasma input source models, understanding of facility interactions, and validation with flight measurements and ground testing. To date, comparisons of plume profiles between ground testing, flight data, and/or simulations demonstrate inconsistent agreement [33- $\underline{37}]$.

\section{Experimental Apparatus}

The test hardware and configuration requirements for plume measurements of ion current density include the Faraday probe, motion control system, power electronics, and data acquisition (DAQ) system to monitor collected current. The power electronics, experimental configuration, and probe calibration considerations are described in the following sections. 


\section{A. Hemispherical Coordinate System and Test Configuration}

Faraday probe measurements in a hemispherical coordinate system are shown in Fig. 7 with respect to Cartesian coordinates, where $\theta$ is the angular position from thruster centerline, $\Phi$ is the azimuthal angle, $R$ is the measurement distance, and $D_{T}$ is the thruster diameter. In this paper, $D_{T}$ is defined as the outer grid diameter for GITs or outer channel diameter for HETs. Different definitions, such as the midchannel diameter of HETs, may necessitate minor modification to equations and will be identified in the text. The plume is assumed axisymmetric about the $z$ axis, and the probe axis of rotation is typically the $y$ axis at the intersection of the thruster exit plane and the thruster centerline axis in Fig. 7. The Faraday probe is swept in an arc at constant $R$, and the collector face is pointed toward the rotation axis at all angular locations of the measurement.

Note that, in a true hemispherical coordinate system, the angular coordinate $\theta$ cannot be negative with respect to the thruster centerline axis. However, for EP thruster testing, probes are generally swept from one side of the thruster to the other. Plasma measurements are collected during the entire sweep, thereby providing data from two opposing azimuthal angles (i.e., $\Phi=0$ and $180 \mathrm{deg}$ ). Common convention within the EP test community is to refer to these two datasets as the positive $\theta$ and negative $\theta$ datasets, where $-\theta$ refers to data collected at $\Phi=180 \mathrm{deg}$ since $\cos (180 \mathrm{deg})$ is -1 . Collecting data in this fashion has the advantage of allowing researchers to determine the symmetry of the plasma plume in the swept plane. For the purpose of this paper, $-\theta$ positions represent the left side of the plume where $\Phi=180 \mathrm{deg}$ and is understood to represent angular positions opposite of the azimuthal plane at $\Phi=0 \mathrm{deg}$.

The hemispherical coordinate geometry should be used for measurements of the far-field HET and GIT plume, which is defined as the region where $R$ is greater than four thruster diameters

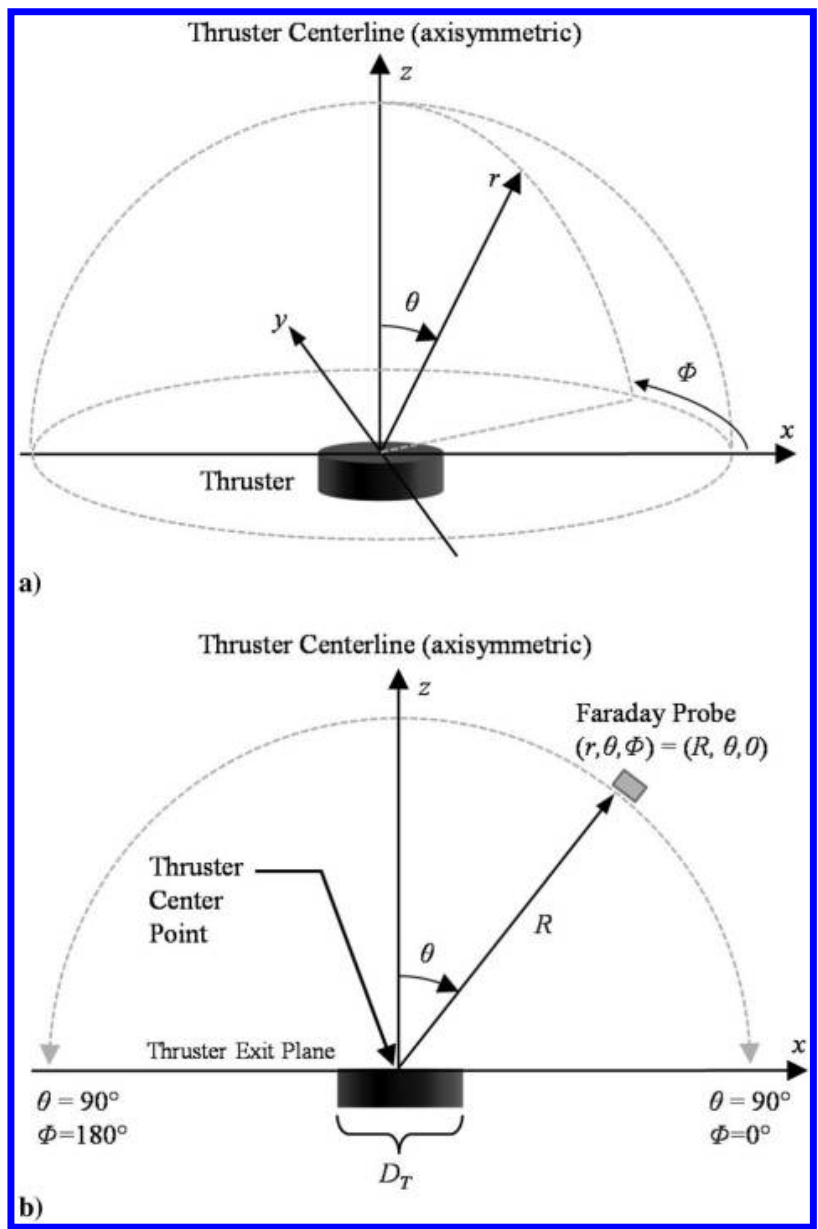

Fig. 7 Diagram of a) hemispherical coordinate system for far-field Faraday probe measurements and $b$ ) measurement geometry in $x-z$ plane where $\Phi=0$ and $180 \mathrm{deg}$. downstream (TDD) of the exit plane, such that $R / D_{T}$ is greater than 4 . Measurements where $R / D_{T}$ is less than 4 have been shown to introduce systematic error, since the hemispherical coordinate system assumes a point ion source [18]. This issue is further discussed in Sec. V.B. In addition, multiple HET investigations have shown a nonnegligible population of beam ions on the periphery of the plume beyond $\theta=50 \mathrm{deg}$ for different thruster designs and over a wide range of operating conditions [38,39]; the hemispherical coordinate system is well-suited to evaluate these divergent plume structures.

If an external cathode is used for beam neutralization and the Faraday probe measurement sweep is conducted in the $\Phi=0$ deg plane, the cathode should be positioned in the $\Phi=90 \mathrm{deg}$ or 270 deg plane (i.e., $\pm y$-axis). Faraday probe sweeps in the cathode plane may measure localized plume asymmetry near the cathode.

\section{B. Cylindrical Coordinate System and Test Configuration}

The cylindrical coordinate system is defined in Fig. $\underline{8}$ with respect to Cartesian coordinates, where $r$ is the radial coordinate, $z$ is the axial coordinate, and $\Phi$ is the azimuthal angle [40]. The plume is assumed to be axisymmetric about the $z$-axis and the axial measurement distance $Z$ is defined with respect to the thruster exit plane, where $z=0$. Beam ions are assumed to be aligned in the axial direction, and therefore the probe face is oriented normal to the $z$-axis. The probe is typically swept in the radial direction at a fixed axial distance $Z$, with $\Phi=0 \mathrm{deg}$ and/or $180 \mathrm{deg}$.

In a true cylindrical coordinate system, $r$ cannot be negative with respect to the thruster centerline axis. Similar to discussions of $-\theta$ in the hemispherical coordinate system, near-field Faraday probe measurements are typically swept across the plume. Thus, a single

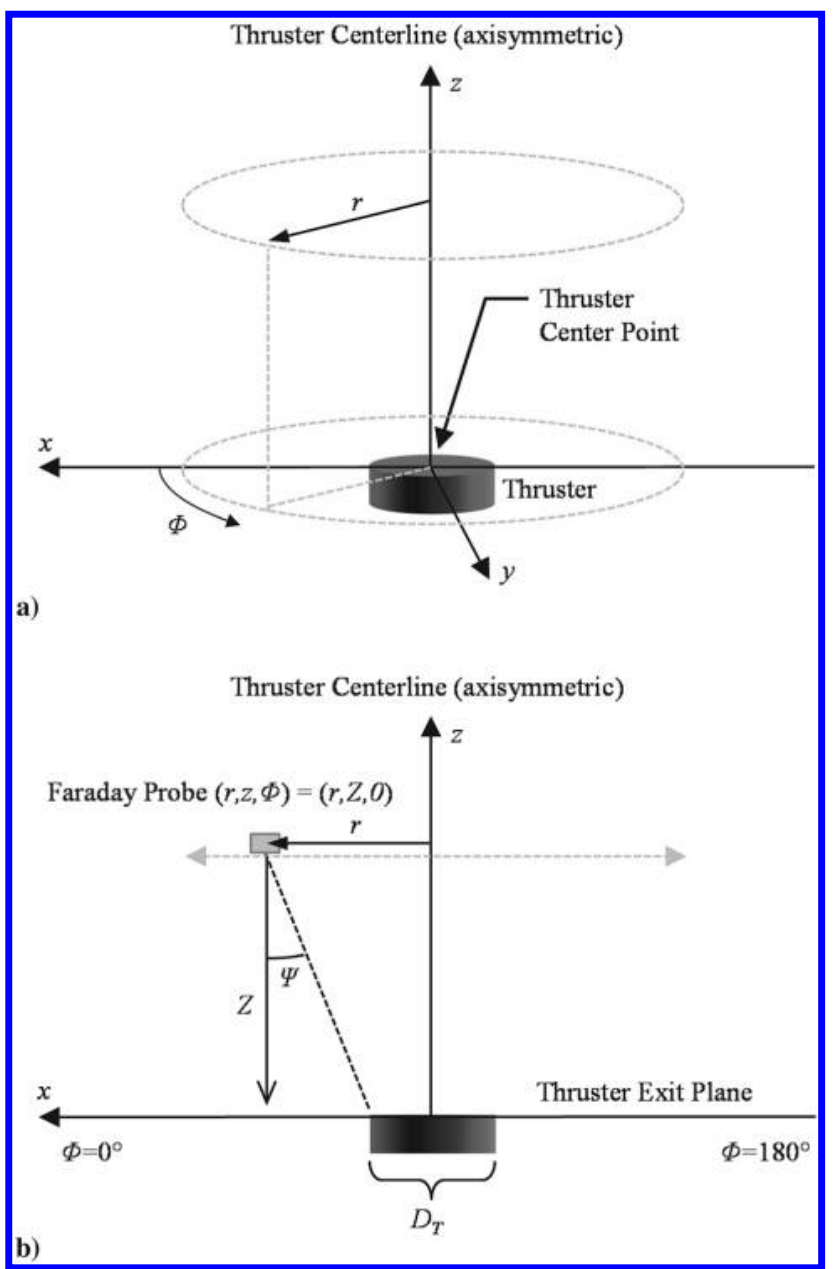

Fig. 8 Diagram of a) cylindrical coordinate system for Faraday probe measurements and b) measurement geometry in $x-z$ plane where $\Phi=0$ deg. 
dataset includes opposing azimuthal angles (i.e., $\Phi=0$ and $180 \mathrm{deg}$ ). Common convention within the EP test community is to refer to these two datasets as the positive $r$ and negative $r$ datasets, where $-r$ refers to data collected at $\Phi=180$ deg because $\cos (180 \mathrm{deg})$ is -1 . Collecting data in this fashion has the advantage of allowing researchers to assess plume symmetry. For the purpose of this paper, $-r$ positions represent the right side of the plume where $\Phi=180 \mathrm{deg}$ and are understood to represent angular positions opposite of the azimuthal plane at $\Phi=0 \mathrm{deg}$.

A cylindrical coordinate system should be used in the near-field plume extending from the thruster exit plane to $\mathrm{Z} / D_{T}$ less than four, which is equivalent to $R / D_{T}$ less than four on the thruster centerline in the hemispherical coordinate system. If an external cathode is used for beam neutralization and the Faraday probe measurement sweep is conducted in the $\Phi=0 \mathrm{deg}$ and/or $180 \mathrm{deg}$ plane, the cathode should be positioned in the $\Phi=90$ or $270 \mathrm{deg}$ plane (i.e., $\pm y$ axis). Faraday probe sweeps in the cathode plane may measure localized plume asymmetry and plasma gradients near the cathode, which complicate data analysis [41].

A limitation of the cylindrical coordinate system is the inability to measure high-energy beam ion flux at high angles from the thruster centerline approaching $\Psi=90 \mathrm{deg}$ (in Fig. 8) because the probe is oriented perpendicular to the thrust axis. HETs in particular exhibit a nonnegligible fraction of beam ions on the plume periphery [38, 42]; thus, the cylindrical coordinate system may not be practical for quantitative evaluation of the near-field HET plume for $1<\mathrm{Z} / D_{T}<4$.

\section{Probe Positioning Techniques}

Faraday probe measurement positioning may be performed with two methods, either 1) maintain continuous motion and data acquisition, or 2) stop at a specific measurement position, record data, and then move to the next position. Probe acceleration and translation speed impacts spatial accuracy, resolution, and scan duration. Large acceleration and deceleration magnitude may induce probe vibration, and thus it is critical to characterize the effect on resolution if the probe is stopped at each measurement location. For continuous motion, the probe translation speed will limit spatial measurement resolution. Limiting factors for slow translation speeds include long duration of the measurement sweep, probe heating, and survivability. High-speed motion stages should be employed if excessive probe heating and survivability are an issue, which imposes additional demands on the mechanical setup. For example, the supporting structure for Faraday probe armature and the high-speed motion stage must be secure, and they should be attached to the test facility in a way that also braces against possible torsional modes. Additional information on highspeed probe positioning may be found in $[43,44]$.

There is currently no simple formula for determining a minimum motion stage speed necessary to pass through the thruster plasma. The probe area and the residence time in the plasma are proportional to the total energy transferred to the probe as heat. Past near-field studies of a $20 \mathrm{~kW}$ HET plasma demonstrated a probe residence time in the plasma of $\sim 1 \mathrm{~s}$ was acceptable for a probe with frontal area of $\sim 10 \mathrm{~mm}^{2}$ at 0.05 TDD from the exit plane [45]. During these studies, the probe translation speed ranged from 150 to $500 \mathrm{~mm} / \mathrm{s}$.

\section{Power Electronics and Data Acquisition}

Power electronics serve two functions: to source electrode bias voltage, and to measure ion current collected by the electrode. The probe bias repels electrons such that the probe is in ion saturation. Probe electrode biasing is accomplished through the use of one or more dc power supplies, typically -15 to $-30 \mathrm{~V}$ with respect to facility ground in far-field measurements and approaching $-100 \mathrm{~V}$ with respect to facility ground in near-field measurements. A single power supply can bias the collector and guard ring electrodes to the same bias potential $V_{\text {bias }}$, assuming equivalent electrical wiring lengths are used to both electrodes. Additional power supplies may be necessary if a filtering or collimating technique is used that requires different voltages than that applied to the collector. The electrical diagram of a recommended probe circuit is shown in Fig. 9, where the ion current to the collector $I_{\mathrm{FP}}$ may be read as a voltage $V_{\mathrm{FP}}$ across the

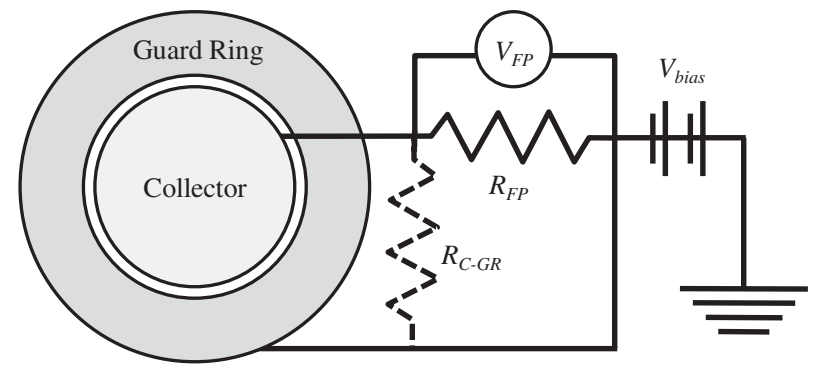

Fig. 9 Electrical schematic of a typical Faraday probe showing the collector and guard ring.

resistor $R_{\mathrm{FP}}$. The resistance of $R_{\mathrm{FP}}$ is typically between 10 and $1000 \Omega$. Larger $R_{\mathrm{FP}}$ resistance values may influence measurements in several ways, including generation of a low-pass filter, creating a voltage divider effect with measurement equipment, or JohnsonNyquist noise [46]. A voltmeter may be used to measure $V_{\mathrm{FP}}$. Often, a DAQ system or digital multimeter with high impedance (greater than gigaohms) is required to further reduce leakage current. (Leakage current is equivalent to probe voltage divided by input impedance. For megaohm input impedance, the leakage current may exceed a nonnegligible fraction of the probe signal strength in the far-field plume $\left(\sim 10-100 \mu \mathrm{A} / \mathrm{cm}^{2}\right)$, thereby limiting resolution and increasing measurement uncertainty. For example, a probe bias of $-20 \mathrm{~V}$ and $1 \mathrm{M} \Omega$ input impedance generates leakage current of $\sim 20 \mu \mathrm{A}$, whereas $1 \mathrm{G} \Omega$ input impedance corresponds to $\sim 20 \mathrm{nA}$.)

Resistance between the collector and guard ring, identified as $R_{\mathrm{C}-\mathrm{GR}}$ in Fig. 9 , and between the collector to ground should be large, such that stray current does not exceed the ion current measurement resolution. For ion current measurement resolution of $1 \mathrm{nA}$ and typical electrode bias voltage ranging from -10 to $-100 \mathrm{~V}$, the resistance $R_{\mathrm{C}-\mathrm{GR}}$ from the collector to the guard ring should exceed $100 \mathrm{M} \Omega(=10 \mathrm{~V} / 1 \mathrm{nA})$. An isolation amplifier may be implemented to prevent arc events from damaging the DAQ system.

The measurement DAQ rate is typically determined by the desired spatial resolution and probe speed. High-speed probe positioning may require an encoder. If an encoder is not used, the repeatability of probe positioning should be characterized to evaluate precision and estimate positioning error. During measurements with low data acquisition rates, the probe location may be monitored through encoders, through the motion controller, or through the controlling program.

\section{E. Probe Calibration, Alignment, and Periodic Maintenance}

The methods described in this section are suitable to minimize systematic error in key aspects of the measurement system and experimental configuration in a ground test environment. The Faraday probe, probe positioning, and DAQ system may be characterized and/or calibrated according to manufacturer recommendations before Faraday probe measurements. Ideally, before testing the probe, an experimental measurement system would be evaluated with an ion source that could provide a uniform, monoenergetic ion beam with the ion energy per charge ranging from $0 \mathrm{~V}$ to greater than the maximum thruster acceleration voltage. Although this approach may be ideal in an investigation with unlimited resources, there are significant limitations in applying the characterization of an ideal ion beam to measurements of EP thruster plumes and mitigating systematic error associated with facility pressure effects. First, the thruster ion beam will vary spatially (and temporally in the case of HETs) throughout the plume, including ion energy distribution and ion charge states. Second, the thruster ion beam will be influenced by facility effects and vary with thruster operating condition. Thus, the complexities of EP plumes and resources required to characterize the experiment with an ion source make it impractical for most investigations. In practice, the errors may be characterized and/or mitigated with careful probe design, using correction factors, and implementing the test and analysis methods described in Secs. IV and V.

The manufacturer uncertainty in DAQ system components should account for many drift errors or offsets. It is important to account for 
DAQ system error for the measurement range and DAQ thermal environment specified by the manufacturer. In addition, a null measurement of the DAQ system without plasma may be used to assess DAQ system uncertainties.

Resistance of the collector to ground and $R_{\mathrm{C}-\mathrm{GR}}$ should be measured before testing; both should exceed $100 \mathrm{M} \Omega$. If the resistance is below this threshold, the exposed surfaces within the gap between the collector and guard ring should be evaluated for surface deposition. Faraday probe contaminants may include materials used in the thruster, cathode, spacecraft or facility, Faraday probe materials, and experimental mounting hardware. Common sputtering contaminants may include iron, tungsten, molybdenum, stainless steel, ceramics (i.e., boron nitride, alumina, etc.), graphite, and Kapton. Sputtering of dielectric or conductive materials may impact probe ion collection in different ways, such as leakage current from the collector, collector thermionic emission, or collector secondary electron emission (SEE) [47-50]. Contamination effects will likely manifest in multiple ways and may affect the data in a competing manner. In this case, the probe should undergo a cleaning process to reduce contamination and remove buildup of sputtered material through exposure to high-energy ions in the beam [ $\underline{51}, \underline{52}]$, or it may be cleaned periodically, such as with $40 \%$ isopropyl alcohol. Although it is possible to remove surface material buildup, and even achieve a polished finish by sanding the probe surfaces, this should be done with caution to avoid altering probe dimensions and tolerances; reassessment of probe dimensions and evaluation of the collection area are recommended if sanding is implemented.

Probe alignment and orientation with respect to the thruster should be conducted before evacuating the facility. For evaluation of the axisymmetric plume, the collector face should be aligned parallel to the thruster exit plane to within $1 \mathrm{deg}$ when the probe is located on the thruster centerline. The downstream distance $R$ (or $Z$ ) should be measured along the thruster centerline from the centerpoint of the probe collector surface to the intersection with the thruster exit plane, as shown in Figs. 7 and $\underline{8}$. The definition of the thruster exit plane is thruster design dependent, and it is left to the user. The probe axial and radial position accuracy should be within $1 \mathrm{~mm}$ or $0.5 \%$ of $R$ (or $Z$ ) at the furthest measurement distance (i.e., $5 \mathrm{~mm}$ at $R=1 \mathrm{~m}$ ): whichever is greater. Alignment may be repeated at multiple distances and angles from the thruster centerline. These alignment tolerances are less than the measurement resolution, and they are intended to minimize spatial error in measurements to provide sufficient resolution for plume regions with steep gradients in plasma properties and for validation of high-fidelity modeling and simulation $[53,54]$. Due to the possibility of damage to the probe or thruster with misalignment, a device for alignment while under vacuum may be used, such as a contact probe and a reference with a fixed, known position relative to the thruster.

During plasma plume mapping, there should be no obstructions in the line of sight of the Faraday probe to any point of the thruster plasma discharge. Diagnostic wiring should be a coaxial cable or twisted shielded pair, and electrical connections should not be exposed to the plasma. Cable shielding should be grounded to the facility walls in ground testing. All probe mounting structure and cables near the probe should be downstream of the probe collection surface. Any mounting structure that experiences direct beam ion impingement should be shielded with low-sputter materials, such as Kapton or graphite [48,55].

The user should consider Faraday probe distance from the facility walls or other grounded surfaces. One possibility is maintaining a probe distance greater than one mean free path from the facility walls. In this case, using Eq. (1) for a thruster operating at $1.3 \times$ $10^{-5}$ torr-Xe and a local electron temperature of $2 \mathrm{eV}$ (electronneutral elastic collision cross section of $1.6 \times 10^{-19} \mathrm{~m}^{2}$ ) results in an electron-neutral MFP of $12.3 \mathrm{~m}$ [56]. Thus, this approach is likely not practical. An alternative approach is based on the Debye length $\lambda_{D}$ in Eq. (2), which is the length scale that the bulk plasma shields the effect of the perturbing electrode:

$$
\lambda_{D}=\sqrt{\frac{\varepsilon_{0} k_{B} T_{e}}{e^{2} n}}
$$

Electron number densities typically vary over more orders of magnitude than electron temperature in the EP plume; therefore, the electron Debye length is primarily dictated by the plasma density. For a typical thruster plasma with an electron temperature of $2 \mathrm{eV}$ and an electron number density of $10^{13} \mathrm{~m}^{-3}$, the electron Debye length is $3.3 \mathrm{~mm}$.

\section{Test Methodology}

\section{A. Test Conditions and Plasma Regimes}

Spatial variations of plasma properties in EP plumes span orders of magnitude, and they necessitate consideration of the facility interactions with the thruster plasma and probe ion collection. The time-averaged ion current density in the plume of HETs and GITs typically ranges from 1 to $100 \mathrm{~mA} / \mathrm{cm}^{2}$ in the near-field to less than $0.001 \mathrm{~mA} / \mathrm{cm}^{2}$ in they periphery of the far-field plume $[21,45]$. Over this region, the time-averaged electron temperature and ion density range from approximately $10 \mathrm{eV}$ and greater than $10^{18} \mathrm{~m}^{-3}$ in the near-field $\left(\mathrm{Z}>0.2 D_{T}\right)$ to less than $1 \mathrm{eV}$ and $10^{13} \mathrm{~m}^{-3}$ in the far-field plume periphery.

Analyses of collisional processes in the plasma plume indicate CEX collisions between beam ions and facility neutrals dominate over ion-neutral momentum collisions or Coulomb interactions [39]. There is an important distinction in CEX collisions between beam ions with facility neutrals or beam ions with non-ionized propellant from the thruster and cathode. The former are a leading source of facility effects that should be evaluated and corrected for. The latter CEX collisional processes exist on orbit and should not be filtered from Faraday probe measurements. Differentiating these CEX processes and characterizing the facility influence on ion beam properties requires the lowest facility pressure achievable and execution of the methodologies described in this paper.

Facility pressure during Faraday probe measurements should be as low as possible to better replicate the space environment. Modern vacuum facilities may achieve background pressure less than $10^{-5}$ torr-Xe. Lower pressure will give more accurate results, and therefore it is highly recommended for model validation data and predictions of the in-space plume properties. The pressure should be monitored near the thruster and adhere to recommendations in [57].

The thruster discharge should reach operational steady state before conducting Faraday probe measurements. Thruster input parameters are typically fixed, such as applied magnetic fields and voltage applied to electrodes. In addition, the thruster should be monitored for possible perturbation during a sweep, which may be associated with plasma interaction with the probe mounting structure or the influence of probe bias and local sheath effects. The definition of steady state and perturbation should be identified by the user. It is recommended that the thruster discharge telemetry be monitored throughout plume characterization. Monitoring may include time-resolved and timeaveraged thruster telemetry such as discharge voltage(s) and current(s), mass flow rate, cathode to ground potential, and thrust.

Facility background pressure may influence the ratio of thruster discharge current to the anode propellant flow rate. If Faraday probes are used to measure the ion current density of the HET plume at multiple background pressures, either the thruster discharge current or anode mass flow rate should be held constant; this may require adjustment of the unfixed parameter and should be reported.

\section{B. Probe Operating Characteristics}

The bias potential to the collector and guard ring should be equal, and it must be sufficient for collector ion current saturation throughout the plume measurement region. The probe ion saturation should be characterized at multiple locations in the plume to span the maximum and minimum plasma densities and electron temperature. In far-field measurements, this typically corresponds to the minimum $R$ at $\theta=0 \mathrm{deg}$ and the maximum $R$ at $\theta=90 \mathrm{deg}$. In cylindrical coordinates, this typically corresponds to the minimum $Z$ downstream of the plasma discharge (e.g., channel centerline for HET) and at maximum distance on the plume periphery (i.e., maximum $Z$ and/ or $r$ ). At each location, the collector ion current should be assessed, starting from $0 \mathrm{~V}$ to a negative bias voltage where the collected 


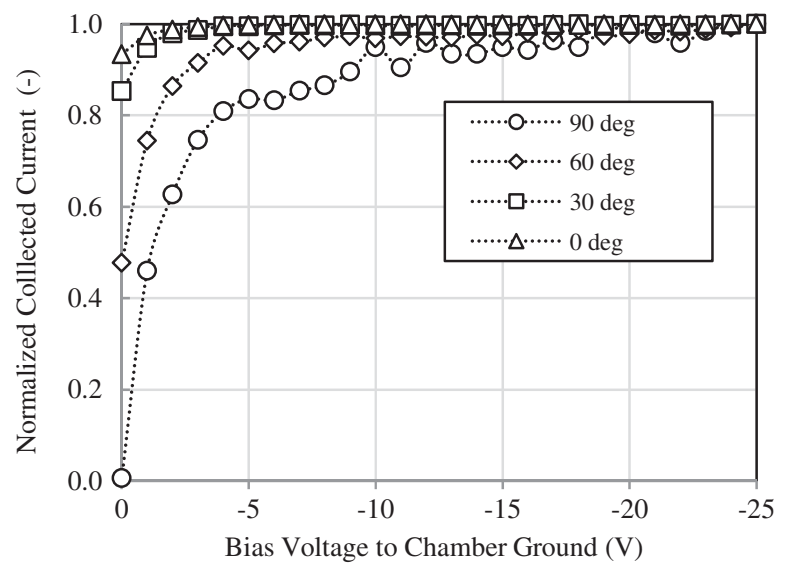

Fig. 10 Normalized Faraday probe collector current in the plume of a 200 W HET at $R=50 \mathrm{~cm}$.

current asymptotes. This negative bias voltage indicates ion saturation. Characterization of ion saturation is demonstrated in Fig. 10 at $R=50 \mathrm{~cm}$ for the nested Faraday probe described in [18], where the measured collector current is peak normalized at each angular location. There is not a quantified value for the degree of ion saturation necessary for Faraday probes in an EP plasma. Thus, it is suggested that ion saturation is achieved when the change in measured collector current is less than approximately $1 \%$ per volt of collector bias. Applying this criterion to data in Fig. 10, ion saturation is achieved for an approximately $-20 \mathrm{~V}$ bias on the collector and guard ring with respect to test facility ground. This value is consistent with bias voltage typically used in far-field measurements, which range from -10 to $-30 \mathrm{~V}$. Typically, the least negative bias voltage necessary to achieve ion saturation throughout the far-field plume should be used. In near-field measurements, there can be a large variation in bias voltage required for ion saturation, and interactions between the plasma and thruster surfaces can introduce large variations in floating potential. A Faraday probe bias voltage as low as $-100 \mathrm{~V}$ may be necessary to enter the ion saturation regime at the closest approach to the thruster [45]. Since the bias voltage can vary greatly depending on distance from the thruster and thruster operating condition, a variable bias voltage may be used. If a fixed Faraday probe bias voltage is used at all locations, local Langmuir probe plasma measurements may inform whether collector sheath expansion is impacting Faraday probe measured current.

The wide range of plasma conditions in the EP plume necessitate careful evaluation of collected ion current due to ambient plasma and the collector plasma sheath. The Bohm sheath criterion is used to calculate the probe ion current (also called the Bohm current [6]) in Eq. (3), where the thin-sheath assessment is applicable $\left(R_{P}>10 \bar{\lambda}_{D}\right)$ [58]:

$$
j_{\mathrm{Bohm}}=\mathrm{e}^{\frac{-1}{2}} e n \sqrt{\frac{e T_{e}}{M_{i}}}
$$

The Bohm current density $j_{\text {Bohm }}$ is evaluated for $n$ and $T_{e}$ at the edge of the presheath. It is expected to increase for elevated background pressure; thus, the Faraday probe would measure an artificially high ion current density. If the ratio of $R_{P} / \lambda_{D}$ is less than 50, the collector sheath will expand as an oblate ellipsoid and increase the effective ion collection area $[\underline{59}, 60]$. Note that the outer diameter of the biased surface is suitable for evaluation of sheath expansion; thus, probe radius $R_{P}$ is equal to the guard ring outer radius or else $R_{P}$ is equivalent to $R_{C}$ if no guard ring is used. Probe sheath expansion is most likely to arise for a small probe radius on the plume periphery at low background pressure, where the plasma density is lowest. Trends in $j_{\text {Bohm }}$ and $R_{P} / \lambda_{D}$ are evaluated in Fig. 11 over a range of typical plasma properties, where $R_{P}=20 \mathrm{~mm}$. In Fig. 11, $j_{\text {Bohm }}$ shows significant variation over the range of plasma density from $10^{13}$ to $10^{16} \mathrm{~m}^{-3}$ but minor sensitivity to electron temperature from 0.5 to $3 \mathrm{eV}$. For a typical Faraday probe radius of $20 \mathrm{~mm}$ in $3 \mathrm{eV}$ plasma conditions, the thin-sheath assessment is not

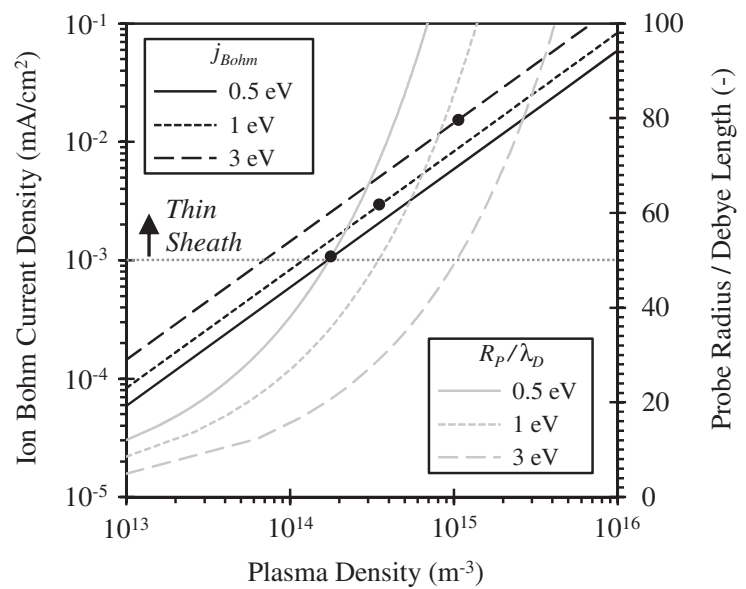

Fig. 11 Trends in ion Bohm current density and ratio of probe radius $\left(R_{P}=20 \mathrm{~mm}\right)$ to Debye length.

applicable (i.e., $R_{P} / \lambda_{D}<50$ ) at pressure less than approximately $10^{15} \mathrm{~m}^{-3}$ (in Fig. 11); thus, collector sheath expansion may be an issue at these local plasma conditions. In cases where $R_{P} / \lambda_{D}$ is less than 50, the trends in ion Bohm current may deviate from Eq. (3), as identified with the black circles on lines of $j_{\text {Bohm }}$ in Fig. 11 for $R_{P}=20 \mathrm{~mm}$. The other issue identified in Fig. 11 is elevated ion Bohm current density at high plasma density, which may be a nonnegligible fraction of the ion beam current. Ideally, $j_{\text {Bohm }}$ should be less than $1 \%$ of the measured ion current density. Issues associated with probe sheath expansion and Bohm current collection may have impacted data in Secs. V.B and V.E, and they will be identified. To evaluate these issues and reduce measurement error, Langmuir probe measurements of local plasma density and electron temperature near the Faraday probe may be used to quantify the effects.

In general, the high-energy flux of EP plasma plumes generates a harsh environment for plasma diagnostics, the thruster, and other exposed surfaces in the test or operational space environment. Probe surfaces with long-duration exposure to beam ions may undergo degradation through sputtering and subsequent deposition of nearby materials. This could impact the probe collection area, collector surface properties, or electrical characteristics of the probe. Thus, it is recommended to conduct visual inspection and electrical verification of the Faraday probe at the beginning and end of a test campaign, or if there is a change in measurement repeatability over time.

In addition to surface degradation, ions transfer a significant fraction of their energy upon probe impact, leading to elevated temperatures of the probe, associated support structure, and wiring. For modern HET and GIT designs of moderate power (less than $5 \mathrm{~kW}$ ), the maximum ion current density in the near-field is typically less than $250 \mathrm{~mA} / \mathrm{cm}^{2}$ for HETs and less than $10 \mathrm{~mA} / \mathrm{cm}^{2}$ for GITs $[21,44,54,61]$. For a beam where each ion deposits $400 \mathrm{eV}$, the corresponding maximum energy flux is typically less than $100 \mathrm{~W} / \mathrm{cm}^{2}$ for HETs and less than $4 \mathrm{~W} / \mathrm{cm}^{2}$ for GITs. Since plasma diagnostics for EP plumes rarely have active cooling, the principal means of heat rejection is radiation and passive thermal conduction through the probe support structure. In the worst-case estimate of heating with no thermal conduction, the collector radiated power per unit area is calculated with the Stefan-Boltzmann relation as $\varepsilon \sigma_{\mathrm{SB}} T_{S}^{4}$, where $T_{S}$ is surface temperature [62]. For a molybdenum collector at $1000 \mathrm{~K}$, the radiated power is approximately 0.6 to $1.8 \mathrm{~W} / \mathrm{cm}^{2}$ for a polished surface $(\varepsilon=0.10)$ or roughened surface $(\varepsilon=0.31)$, respectively [62]. This is significantly lower than the high-energy flux conditions of HETs (less than $100 \mathrm{~W} / \mathrm{cm}^{2}$ ) and GITs (less than $40 \mathrm{~W} / \mathrm{cm}^{2}$ ) described previously; therefore, probe heating is expected unless there is sufficient thermal conduction in the probe apparatus. This will be further considered for higher-energy density devices discussed in Sec. IX, including electromagnetic propulsion concepts.

In plume regions with high-energy flux, probe heating may introduce additional measurement error. Thermionic emission current density may be estimated with the Richardson-Dushman 
equation in Eq. (4), where $\varphi$ is the material work function and the parameter $A_{G}$ is specific to material and surface properties (based on the theoretical Richardson constant) [주:

$$
j_{t}=A_{G} T_{S}^{2} \mathrm{e}^{\frac{-\varphi}{B_{B} T_{S}}}
$$

The Richardson-Dushman equation is valid for surfaces in zero or weak electric fields. Past measurements of the region surrounding a nude Faraday probe in the far-field HET plume revealed local electric fields were less than approximately $100 \mathrm{~V} / \mathrm{m}$ [19], and Eq. (4) is suitable in these plasma conditions. Measurements in regions of high electric field strength may be susceptible to field enhanced thermionic emission, also known as the Schottky effect, and may require a modified version of the Richardson-Dushman equation [64]. A thermionic emission current less than $1 \%$ of the Faraday probe collected current is recommended, and the maximum collector temperature may be estimated accordingly for each experiment. For example, a far-field measurement with an ion current density of $0.01 \mathrm{~mA} / \mathrm{cm}^{2}$ on the plume periphery would correspond to a maximum collector temperature of $\sim 1500 \mathrm{~K}$ for thermionic emission less than $10^{-4} \mathrm{~mA} / \mathrm{cm}^{2}$, based on Eq. (4) for a pure tungsten surface where $A_{G}=60 \mathrm{~A} /\left(\mathrm{cm}^{2} \cdot \mathrm{K}^{2}\right)$ and $\varphi=4.52 \mathrm{eV}[\underline{65}, 66]$. Note that Eq. (4) is sensitive to $\varphi$, which may have a significant error due surface characteristics, such as adsorption of contaminants on the surface [67]. Since the collector surface material properties are typically not well defined, the collector temperature may be monitored with a thermocouple to enable qualitative analysis of thermionic emission. This may also set allowable limits for collector temperature and inform probe positioning speed through the plume. For example, a collector temperature less than $700 \mathrm{~K}$ would maintain thermionic emission below $10^{-4} \mathrm{~mA} / \mathrm{cm}^{2}$, even if surface contamination was suspected of lowering the work function to $\varphi=2 \mathrm{eV}$.

In the absence of collector temperature measurements, the effects of probe heating and measurement drift are often considered by consecutive measurements in opposite sweep directions. The user may determine whether discrepancies between scans are acceptable. For example, a past study showed Faraday probe measurements unaffected by probe heating yielded repeatable profiles of collected current density to within $2 \%$ [18]. In the event ion current density profiles are not repeatable, experience measurement drift, or the measurement exhibits sensitivity to sweep direction, further assessment of the cause is necessary, as this could be associated with effects not related to the Faraday probe, such as thruster construction, experimental misalignment, and plasma dynamics or perturbations.

\section{Far-Field Spatial Range and Resolution}

When using the hemispherical coordinate system for far-field plume measurements, the angular sweep should be performed in a single plane from $\theta=0$ to $90 \mathrm{deg}$ at constant $R$ and fixed $\Phi$ (i.e., $\Phi=0 \mathrm{deg}$ ), according to Fig. 7a. Faraday probe sweeps should also be conducted from $\theta=0$ to 90 deg at fixed $\Phi$ for the opposite side of the plume (i.e., $\Phi=180 \mathrm{deg}$ ), such that a continuous measurement is taken from $\theta= \pm 90 \mathrm{deg}$ at fixed $R$, as shown in Fig. 7b, to assess plume symmetry. Asymmetry in the plume may arise due to thruster misalignment with respect to the coordinate geometry, probe positioning misalignment, or an asymmetric feature of the thruster, such as external cathode placement or a mechanical misalignment in thruster assembly. If significant asymmetry in the plume is observed, the cause and the impact on measurement uncertainty should be evaluated.

The plume periphery beyond $\theta=90 \mathrm{deg}$ may be evaluated to characterize particle scattering, although data are more susceptible to facility effects and may influence calculations of total ion beam current and divergence. Angular measurement resolution should be less than or equal to $2 \mathrm{deg}$, and less than $1 \mathrm{deg}$ is recommended. In addition, the Faraday probe should be sized such that the ratio of probe collector diameter to measurement radius is less than the angular resolution (i.e., $2 R_{C} / R \leq d \theta \leq 2 \mathrm{deg}$ ). Thus, for a measurement resolution of $1 \mathrm{deg}$ at a measurement distance of $100 \mathrm{~cm}$, the probe collector diameter should be less than $1.7 \mathrm{~cm}$.

A distance greater than four TDD should be used for far-field Faraday probe measurements employing the hemispherical coordinate system. This is necessary based on the assumption of an axisymmetric hemispherical coordinate system with a point source origin, where approximating the plasma discharge as a point source is a poor assumption for measurement distances closer than four TDD [18]. Further discussion on the systematic spatial measurement error and analytical corrections are provided in Sec. $\underline{\text { V.B. }}$.

\section{Near-Field Spatial Range and Resolution}

When using the cylindrical coordinate system for near-field measurements, the Faraday probe radial sweep should be performed at constant axial distance $Z$ and fixed $\Phi$ (e.g., $\Phi=0 \mathrm{deg}$ ), as shown in Fig. 8 . The radial measurement range should extend to the location where measured ion current density is less than $0.2 \%$ of the maximum current density along the radial profile at fixed $Z$. This range enables a $0.2 \%$ threshold-based integration limit, and it is recommended based on past experiments [45]. Faraday probe sweeps should also be conducted at fixed $\Phi$ for the opposite side of the plume (i.e., $\Phi=180 \mathrm{deg}$ ), such that a continuous radial sweep is taken to less than $0.2 \%$ of the maximum current density value for the left side $(r>0)$ and right side $(r<0)$, as shown in Fig. 8b, in order to assess plume symmetry. Multiple axial measurement distances may be evaluated for near-field plume asymmetry. Measurement resolution in the radial coordinate should be less than $0.01 D_{T}$ between measurement locations, where the best resolution is limited by the probe collector diameter $2 R_{C}$. In the event this criterion is not practical for small thruster designs or for suitable probe signal strength, the probe collector diameter may be minimized to approach the $0.01 D_{T}$ criteria and the radial distance between measurements may be less than $1 \mathrm{~mm}$ (i.e., $2 R_{C} \leq d r \leq 0.01 D_{T}$ or $\leq 1 \mathrm{~mm}$, whichever is greater).

The near-field HET plume should be analyzed at axial distances upstream of the plume merging region, termed the "transitional region" in [54], where ion beams from opposing sides of the channel merge. Past near-field studies of multiple HET designs found this transitional region was approximately 0.5 to $1.0 \mathrm{TDD}[44,45,54]$. Axial distances less than 0.2 TDD are not recommended due to ionization and acceleration processes that may occur downstream of the HET exit plane, and they have been shown to be influenced by background pressure [68]. In addition, distances within 0.2 TDD of the HET body may perturb discharge plasma behavior or encounter plasma floating potentials on the order of the Faraday probe bias potential, such that the probe is not electron repelling and may be susceptible to sheath effects [54]. It should be noted that regions of plume merging, probe perturbation, and issues due to collector sheaths or ion Bohm current may vary with thruster design, operating conditions, and background environment. Thus, the spatial measurement and analysis range should be evaluated for each experiment, and this will be further discussed in Secs. V.C and V.E. In many cases, these issues are mitigated by avoiding plume regions where the issues are present (i.e., plume merging, probe perturbation) or addressed by monitoring thruster discharge telemetry, described in Sec. IV.A.

Near-field measurements of GIT plumes can be made at distances as close as a few millimeters downstream of the ion optics. Such measurements can be used to study individual beamlets. Near-field measurements of the NASA Evolutionary Xenon Thruster (NEXT) plume revealed beamlets merged within $0.1 D_{T}$ downstream of the exit [21]. In NEXT investigations, the very near-field Faraday probe measurements revealed beamlet merging was a viable indicator of far-field plume divergence. However, the actual location of beamlet merging is in large part a function of the ion optics geometry. For example, investigations of the High Power Electric Propulsion (HiPEP) ion thruster, which has flat rectangular ion optics apertures (40 by $90 \mathrm{~cm}$ ), measured individual beamlets as far as $13 \mathrm{~cm}$ downstream of the grids, which was approximately $36 \%$ of the grid width (analogous to $0.36 D_{T}$ ) [69]. To accurately interrogate the region upstream of beamlet merging in GITs, a small Faraday probe 
collector diameter less than the grid aperture spacing is required and a high radial measurement resolution is necessary to resolve beamlet ion current density profiles. Systematic evaluation of different thrusters and examination of facility interactions in the very near-field of GITs is recommended to establish a minimum measurement distance. The maximum near-field distance for GITs is likely limited by the radial measurement range required to capture a significant fraction of beam ions, where proximity to facility walls may be a factor. Thus, the spatial measurement range should be evaluated for each GIT experiment.

\section{E. Characterization of Facility Effects}

Facility pressure effects associated with background neutral particles are inherent in ground-based EP plumes, and they are unavoidable in both near-field and far-field plume measurements. The following guidelines are based on experiments with multiple thrusters at multiple facilities. Although understanding of facility effects on the near-field plume region is limited, many of the same plasma interactions known to impact far-field measurements are expected, as described in Sec. II.B. Thus, recommendations for nearfield measurements are primarily derived from the current state of knowledge for far-field Faraday probe measurements.

Faraday probe measurements of the far-field and near-field plumes should be conducted at a minimum of four distances. Further, plume measurements at each distance should be conducted at a minimum of four background pressures to enable the extrapolation to spacelike conditions from ground testing, and they should adhere to recommendations in [57]. One of the four background pressures should be the lowest achievable facility pressure during thruster operation. Background pressure variation is often achieved by injecting additional propellant gas into the ground test facility or varying the facility pumping. The maximum background pressure is typically less than $5.0 \times 10^{-5}$ torr-Xe; however, this value may vary with thruster technology, operating condition, and facility pumping capability. In HETs, the facility background pressure may influence thruster operating mode and stability, which is caused by the additional neutral density near the thruster plasma that is not present on orbit $[70,71]$. This behavior has been observed in HETs and may be identified by a large change in discharge oscillation behavior or time-averaged discharge current, voltage, or mass flow rate [그, $\underline{73}]$. Significant changes in HET operation may invalidate the pressure characterization for Faraday probe measurements; therefore, the thruster should be monitored for large changes in operation, as described in Sec. IV.A.

In GITs, the influence of background pressure is evident from the accelerator grid current. In the space environment, the accelerator grid current is primarily associated with collection of low-energy ions generated from CEX collisions between beam ions and the nonionized thruster neutrals that escape through the ion optic grids [23]. The elevated pressure in ground testing introduces additional $\overline{\mathrm{CEX}}$ collisions, and thereby increases GIT accelerator grid current that is not present in flight conditions; the increased GIT accelerator grid current indicates increased CEX ions impinging the grid, which cause significant erosion and eventually form holes through the accelerator grid webbing. At high propellant utilization of $\sim 90 \%$, the space equivalent accelerator grid current ranges between 0.25 to $0.5 \%$ of the total beam current over a range of operating conditions, as shown in Fig. 12 for the NASA Solar Technology Application Readiness (NSTAR) ion thruster on the Deep Space One (DS1) Mission and in ground test facilities $[74,75]$. In general, the ratio of accelerator grid current relative to thruster beam current decreases with decreasing chamber pressure, as shown in Fig. 13 for a $30 \mathrm{~cm}$ GIT at 3.2 A beam current in ground tests at NASA Glenn Research Center (GRC) and Jet Propulsion Laboratory (JPL) [76]. This variation can be used as guidance to determine the maximum facility background pressure used in pressure characterization of GIT plumes. Although a maximum allowable ratio of accelerator grid current to total beam current has not been determined, it is expected to be less than a few percent and may be determined by the user.

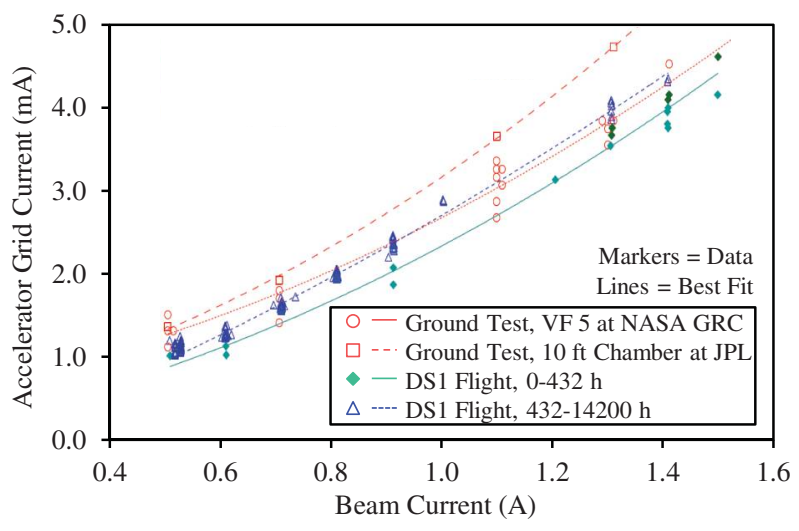

Fig. 12 Accelerator grid current of the NSTAR GIT, recreated from [74].

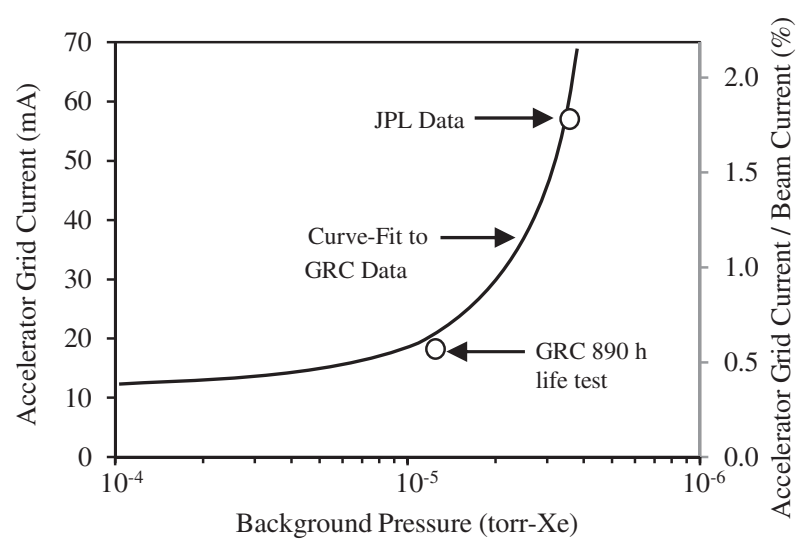

Fig. 13 Accelerator grid current of a $30 \mathrm{~cm}$ GIT, recreated from [76].

Without predictive capability of facility pressure effects on the thruster and on the Faraday probe, the systematic error cannot be determined a priori. Different facility interactions may dominate in different regions of the plume; thus, plume characterization with distance and pressure is considered the best approach to quantify spatial variation in plume properties and probe behavior; systematic errors are not easily quantified through data postprocessing or generalized analysis. Plume characterizations over a range of measurement distances and background pressures are recommended to resolve nonlinear trends, as will be shown for experiments with a $200 \mathrm{~W}$ HET in Sec. V.E. Additional measurement distances, lower facility background pressure, and characterizations at additional pressures will improve corrections for facility interactions and predictions of the space environment.

Qualitative assessment of the plasma profile may be conducted at a single measurement distance or pressure, which limits the application to thruster-to-thruster comparisons of the same model (i.e., acceptance testing) or over the course of an extended firing (i.e., life test). A single Faraday probe measurement should not be used to quantify ion beam current or plume divergence losses in performance. Further, using a single Faraday probe measurement should be avoided for plume predictions of the space environment, comparisons between facilities, or validation data for computational simulations.

\section{Data Analysis}

\section{A. Ion Current Density}

For a Faraday probe collector in ion saturation, the ion charge flux or ion current density $j$ is calculated as

$$
j=\sum_{k} \Gamma_{k} Z_{k} e=\frac{I_{\mathrm{FP}}}{A_{c}+\kappa_{G}} \kappa_{\mathrm{SEE}}
$$

where $\Gamma$ is the local ion flux of the $k$ th species, $Z_{k}$ is the ion charge state, $A_{C}$ is the cross-sectional geometric area of the collector face 
$\left(A_{C}=\pi R_{C}^{2}\right), \kappa_{G}$ is a correction for ions collected in the gap between collector and guard ring, and $\kappa_{\mathrm{SEE}}$ is a correction for SEE at the collector surface. The collected ion current $I_{\mathrm{FP}}$ is a point measurement by the Faraday probe at $(\theta, R)$ for hemispherical coordinates as shown in Fig. 7 or at $(r, Z)$ for cylindrical coordinates in Fig. $\underline{8}$. The correction factors are calculated as

$$
\kappa_{G}=\pi\left(R_{\mathrm{GR}}^{2}-R_{C}^{2}\right)\left(\frac{2 \pi R_{C} h_{C}}{2 \pi R_{C} h_{C}+2 \pi R_{\mathrm{GR}} h_{\mathrm{GR}}}\right)
$$

$$
\kappa_{S E E}=\frac{1}{1+\sum_{k} \frac{\Omega_{k} \gamma_{k}}{Z_{k}}}
$$

where $\gamma_{k}$ is the SEE yield from the $k$ th ion species; $\Omega_{k}$ is the ion current fraction of the $k$ th species; and the parameters $R_{C}, R_{\mathrm{GR}}, h_{C}$, and $h_{\mathrm{GR}}$ are collector and guard ring dimensions for a typical nude Faraday probe configuration, as shown in Fig. 14.

Past experiments demonstrated ions entering the gap between the Faraday probe collector and guard ring are a nonnegligible fraction of the measured collector current [77]. The effect is illustrated in Fig. 14, where the effective cross-sectional ion collection area is the sum of $\overline{A_{C}}$ and $\kappa_{G}$. The correction $\kappa_{G}$ in Eq. (6) is formulated based on the assumption that ions entering the gap volume will be collected by exposed, conductive walls and distributed between the collector and guard ring based on relative wall surface areas. Laboratory studies have shown the assumption is suitable for probes with a nonconducting material at the base of the gap, such as ceramic. Ions entering the gap in probes with a conductive base are preferentially collected by this lineof-sight surface, and the current collected on the sidewalls becomes sensitive to changes in background pressure [77]. The conductive gap base raises issues for facility characterizations, and it is discussed with respect to probe design considerations in Sec. VII.

Although the Faraday probe collector is typically made of low SEE yield material, such as molybdenum or tungsten, SEE has a nonnegligible effect on measured ion current. Secondary electrons

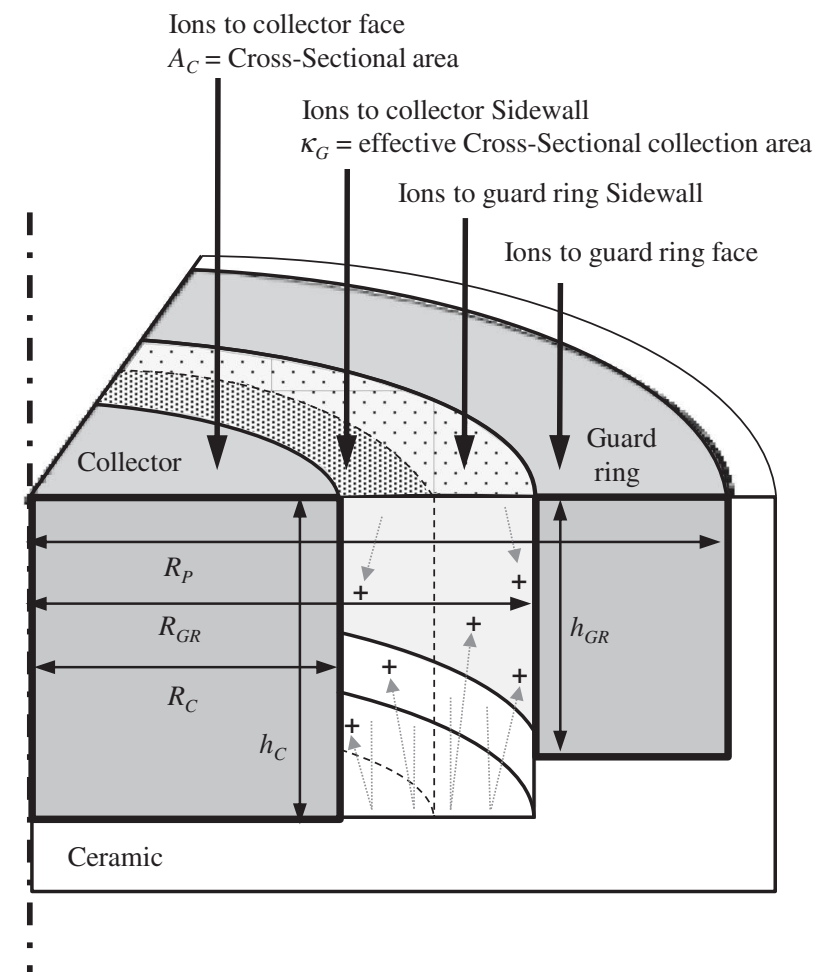

Fig. 14 Illustration of ions collected by the sidewalls of the Faraday probe and the effective increase in cross-sectional ion collection area.
Table 1 Summary of SEE yield for xenon ion bombardment of Faraday probe collector materials [78-80]

\begin{tabular}{lcc}
\hline \hline $\begin{array}{l}\text { Bombarding particle ion } \\
\text { charge state } Z_{k}\end{array}$ & $\begin{array}{c}\text { SEE yield of } \\
\text { molybdenum } \gamma_{k}\end{array}$ & $\begin{array}{c}\text { SEE yield of } \\
\text { tungsten } \gamma_{k}\end{array}$ \\
\hline+1 & 0.022 & 0.016 \\
+2 & 0.20 & 0.20 \\
+3 & $0.70^{\mathrm{a}}$ & 0.71 \\
\hline \hline
\end{tabular}

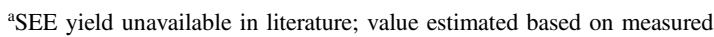
yield for tungsten.

born on the negatively biased collector surface will accelerate away from the probe, which artificially increases measured current. The correction $\kappa_{\mathrm{SEE}}$ formulated in Eq. (7) accounts for the effect, and it may be calculated using the SEE yield values in Table 1 [78-80]. Tungsten and molybdenum SEE yields in Table 1 were averaged for xenon ion bombardment over the energy range of $100-1000 \mathrm{~V}$, where measured yields varied by less than $20 \%$ for $\mathrm{Xe}^{+}$bombardment and less than $10 \%$ for $\mathrm{Xe}^{2+}$ and $\mathrm{Xe}^{3+}$ bombardment. Note the molybdenum SEE yield for $\mathrm{Xe}^{3+}$ ion bombardment is unavailable in the literature, and it is estimated in Table 1 based on trends observed for tungsten. Calculation of $\kappa_{\mathrm{SEE}}$ in Eq. (7) requires knowledge of the ion species composition, which may be measured using analysis techniques for ExB probes (also known as Wien filters) developed in [81]. The effect of $\kappa_{\mathrm{SEE}}$ is small but nonnegligible for the ion species compositions of typical HET and GIT plumes; the value of $\kappa_{\mathrm{SEE}}$ is greater than 0.95 for typical EP plumes and greater than 0.90 if the $\mathrm{Xe}^{+}$ion current fraction is reduced to $60 \%$. Thus, not correcting measurements with $\kappa_{\mathrm{SEE}}$ may lead to overprediction of local ion current density by 5 to $10 \%$, and it should be accounted for in the data analysis and/or error assessment. However, the spatial variation of ion species composition in the central plume is not expected to have a significant effect on the value of $\kappa_{\mathrm{SEE}}$; therefore, a single measurement of ion species current fraction in the central core is sufficient to calculate $\kappa_{\text {SEE }}$ [82] for evaluation of ion beam current and divergence. The population of multiply charged ions may increase beyond the central core; thus, it is advised to incorporate local values of ion species current composition in evaluations of local spacecraft plume interactions on the plume periphery.

\section{B. Far-Field Plume}

Far-field test methodologies described in Sec. IV.C provide the necessary characterization to isolate pressure effects and predict time-averaged ion current density properties in the space vacuum environment. This is achieved by simple linear regression [83] of ion current density as a function of pressure at each location in the plume, as exemplified by far-field measurements of the H6 HET plume in Fig. 15. The linear extrapolations of ion current density to vacuum conditions in Fig. 15 reveal regions where the linear regression slope is positive, negative, and near zero. In this paper, a positive slope refers to an increase of ion current density with increasing pressure, such as $\theta=0,30,40$, and 90 deg in Fig. 15. A negative slope refers to a decrease of ion current density with increasing pressure, such as $\theta=3$ and $15 \mathrm{deg}$. A linear regression slope near zero, such as $\theta=1$ and $24 \mathrm{deg}$, occurs in regions when the extrapolation to zero pressure transitions between positive and negative linear regression slope. The coefficient of determination, termed $R$-squared (no relation to radial measurement distance $R$ ), for each linear extrapolation is calculated as the square of the Pearson product moment correlation coefficient, and it can be interpreted as a metric for how well the linear regression fits the data [83]. The $R$-squared term ranges from zero to one, where a value of unity indicates a perfect fit. Although the linear regression slope is near zero in the transition region and is associated with low coefficient of determination, there is minimal change in ion current density as a function of background pressure, and thus the correction is small. The method of extrapolating ion current density to zero pressure conditions has been conducted with multiple HETs at different facilities $[18,70,84-86]$. Based on the far-field plume processes involved, GIT plumes are expected to follow similar trends. 


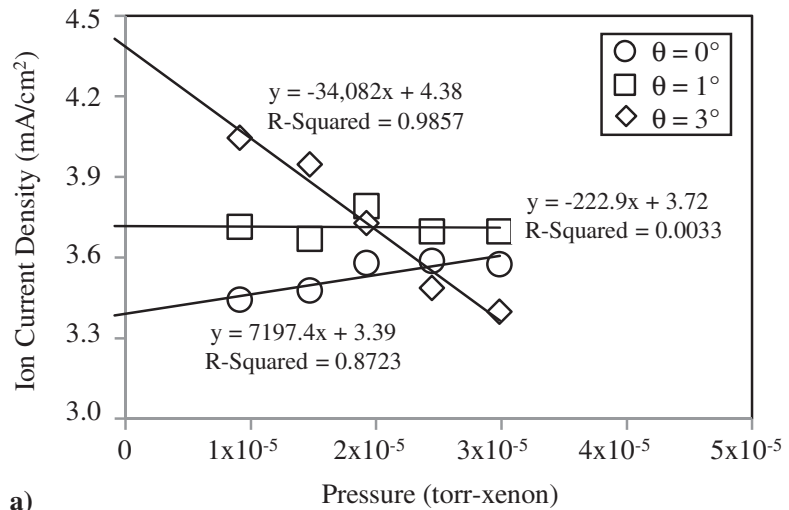

a)
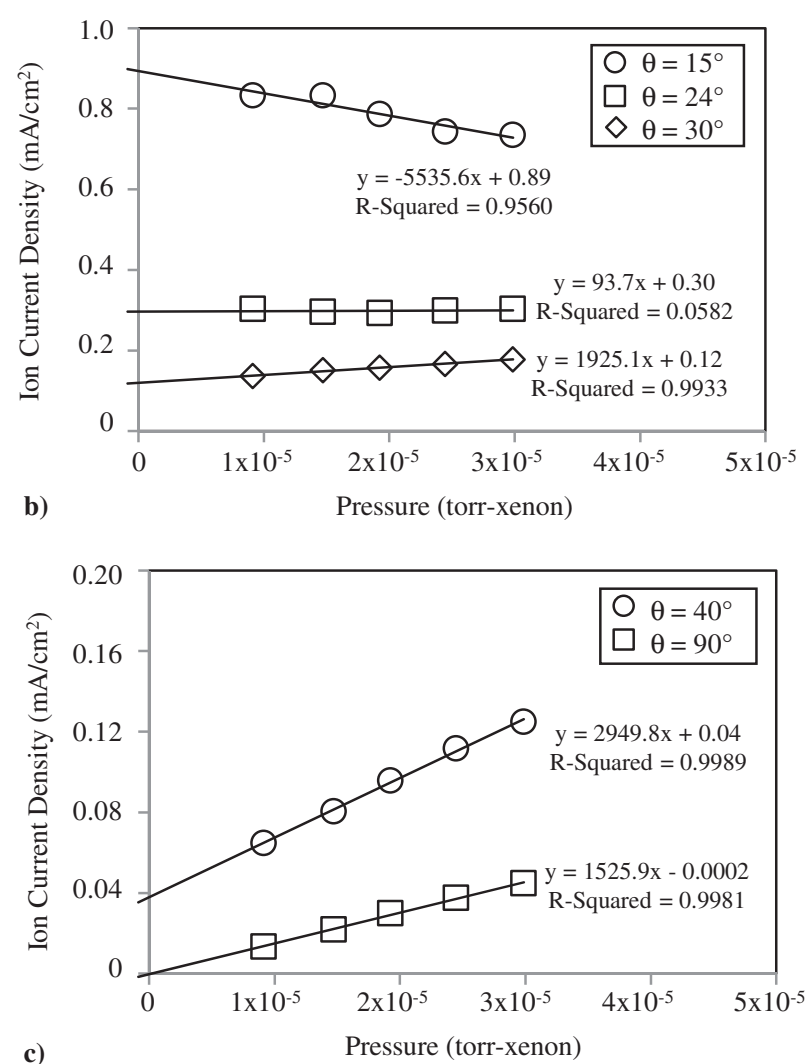

Fig. 15 Ion current density at six TDD as a function of pressure for the H6 HET at $300 \mathrm{~V}, 10 \mathrm{mg} / \mathrm{s}$ anode flow, and $7 \%$ cathode flow.

In Fig. 16, angular profiles of far-field ion current density in the H6 HET plume reveal background pressure effects and show the ion current density linear regression to vacuum conditions at each angular location, based on the method shown in Fig. 15. At the plume periphery in Fig. 16, a difference in ion current density greater than one order of magnitude between low-pressure measurements and extrapolated vacuum conditions is consistent with comparison of flight and ground data for the SPT-100 on the Express Satellite $[33,34,42]$. It should be noted that the linear regression to zero pressure does not arbitrarily force the extrapolated ion current density to zero at $\theta=90 \mathrm{deg}$. The primary sources of error, unrelated to facility effects, in H6 HET results in Figs. 15 and 16 were accounted for, including the effective probe collection area and collector SEE. The effective collection area was determined using Eq. (6), where $\kappa_{G}$ increased the collection area by $13 \%$. The thruster plume was approximately $90 \% \mathrm{Xe}^{+}$and $\kappa_{\mathrm{SEE}}=0.97$ as calculated from Eq. (7). Thus, facility effects were the principal source of error, which is the motivation for facility pressure characterization. Remaining errors were expected to be minimal with respect to the trends in Figs. $\underline{15}$ and 16 .

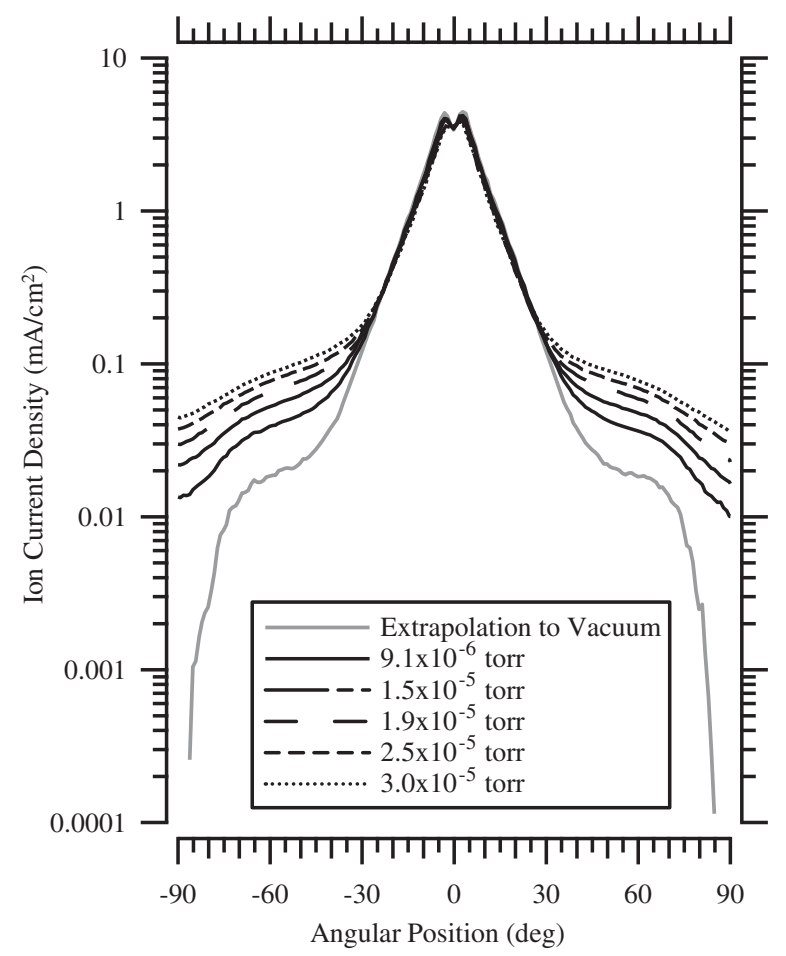

Fig. 16 Ion current density at six TDD as a function of angular position for the H6 HET at $300 \mathrm{~V}, 10 \mathrm{mg} / \mathrm{s}$ anode flow, and $7 \%$ cathode flow.

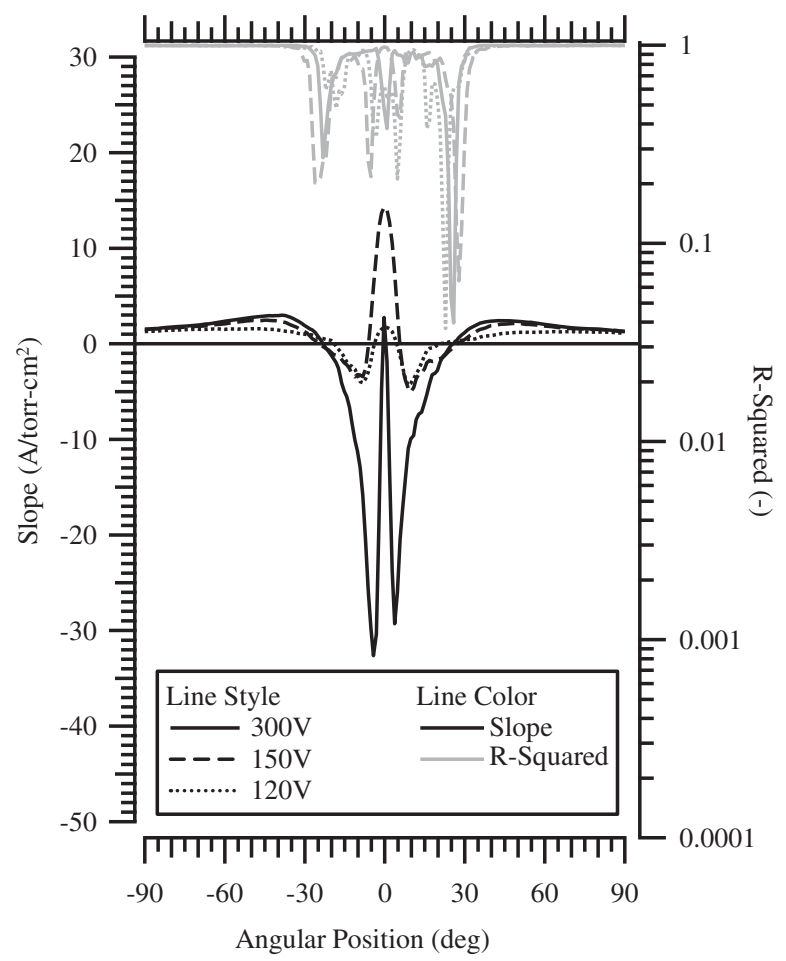

Fig. 17 Linear regression slope and coefficient of determination of the H6 HET ion current density at six TDD.

Figure 17 displays the angular distribution of the linear regression slope and coefficient of determination of the H6 thruster operating at 120,150 , and $300 \mathrm{~V}$ with $10 \mathrm{mg} / \mathrm{s}$ anode flow and $7 \%$ cathode flow. The linear regression slope at thruster centerline decreased from $15 \mathrm{~A} /\left(\right.$ torr $\left.\cdot \mathrm{cm}^{2}\right)$ at $150 \mathrm{~V} \mathrm{H6}$ HET operation to less than $3 \mathrm{~A} /\left(\right.$ torr $\left.\cdot \mathrm{cm}^{2}\right)$ at 120 and 300 V operation. All H6 HET operating conditions showed reduction in ion current density with increasing facility pressure within the central plume core at $\theta= \pm 25 \mathrm{deg}$, where the linear regression slope approached $-30 \mathrm{~A} /\left(\right.$ torr $\left.\cdot \mathrm{cm}^{2}\right)$ for 
the $300 \mathrm{~V}$ condition. The ion current density ranged from approximately 1 to $3 \mathrm{~A} /\left(\right.$ torr $\cdot \mathrm{cm}^{2}$ ) beyond $\theta= \pm 25 \mathrm{deg}$, which is attributed to the net migration of scattered beam ions from the primary ion beam. Although the angular features of the linear regression slope in Fig. 17 are consistent across operating conditions (i.e., positive on thruster centerline, negative in the central core, and positive on the wings), the magnitude and shape are significantly different, thereby revealing the importance of characterizing background pressure interactions.

The linear regression of ion current density and extrapolation to zero pressure are further demonstrated in Figs. 18 and 19 for a $200 \mathrm{~W}$ HET [18]. In Fig. 18, ion current density profiles at 8 and 20 TDD show the influence of background pressure varies with downstream distance and pressure, where ion current density is normalized to the centerline value at eight TDD and $3.1 \times 10^{-6}$ torr-Xe. The effects are also evident in Fig. 19, where a positive linear regression slope on the thruster centerline is attributed to ingestion, ionization, and acceleration of facility neutral particles within $\pm 10 \mathrm{deg}$. The region from \pm 10 to \pm 40 deg in Fig. 19 shows a negative linear regression slope. This feature may be attributed to ion scattering when a slow ion, resulting from a CEX collision event, is accelerated by radial electric fields in the plume. The process leads to increased ion scattering to the plume periphery beyond $\pm 40 \mathrm{deg}$ from centerline and increased overall divergence of the plasma plume.

The angular profiles of linear regression slope were decomposed into contributions from facility neutral ingestion and ion scattering in [18], and they enabled examination of the magnitude and angular range of each effect to enhance evaluations of EP plume structure with variation in pressure, downstream distance, or operating condition. The consistent angular features in the linear regression slope between different thrusters in Figs. 17 and $\underline{19}$ at multiple operating conditions and at multiple downstream measurement distances is evidence that the dominant facility effects are common between the two thruster plumes. Note that ion scattering may be the dominant effect on thruster centerline rather than facility neutral ingestion effects, resulting in a net negative linear regression slope. These results increase confidence that facility effects on the HET ion beam can be effectively characterized and extrapolation of ion current density to vacuum is suitable, given the thruster operation and oscillations are not significantly altered with variation in facility background pressure.

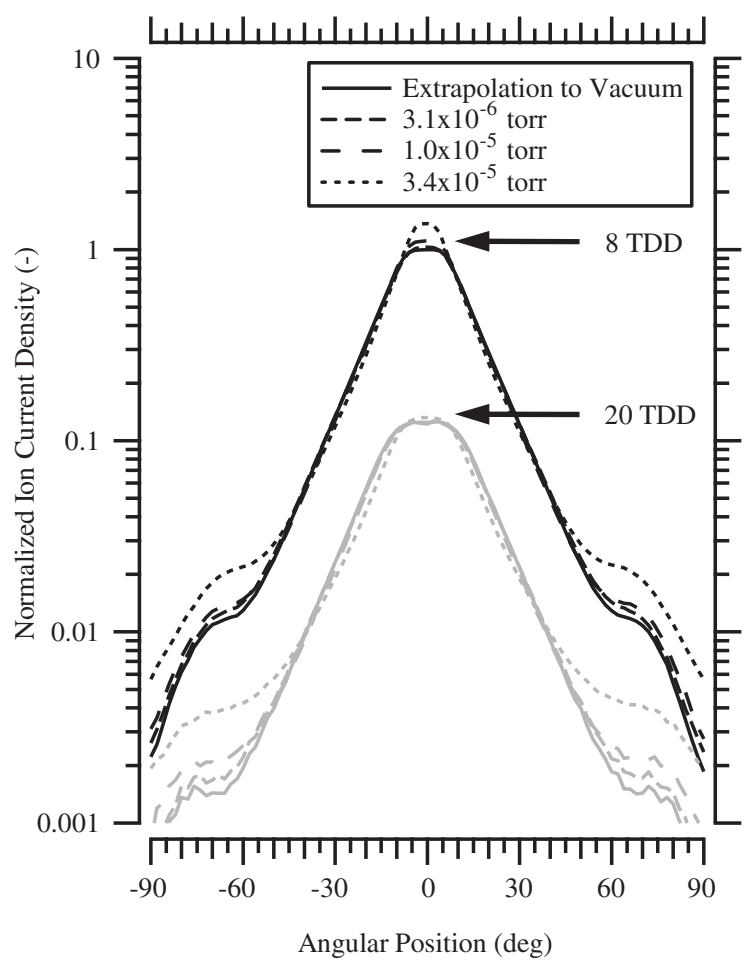

Fig. 18 Normalized ion current density of a 200 W HET.
The far-field GIT beam also shows complex plume structure on the periphery, as shown for the T5 GIT in Fig. 20 [87] at $11.8 \mathrm{~m}$ downstream (11.8 TDD) and $2 \times 10^{-6}$ torr-Xe. Similar structures were observed on the NEXT thruster [21]. As discussed in Sec. V.D, the integrated ion beam current from GIT Faraday probe measurements is often greater than beam current measured by the ion optics. This may be associated with not using corrections described in Sec. V.A, such as $\kappa_{G}$ for the effective probe collection area. To remedy this discrepancy and improve evaluation of GIT beam divergence, a systematic investigation of the GIT far-field plume and characterization with background pressure is warranted, similar to investigations of the $200 \mathrm{~W}$ HET plume in Figs. 18 and 19.

The ion beam current of GITs and HETs is calculated from far-field plume measurements of ion current density in Eq. (5) integrated over the axisymmetric, hemispherical surface for the fixed sweep radius $R$ :

$$
I_{\text {Beam }}=2 \pi R^{2} \int_{0}^{\pi / 2} j[\theta] \frac{\kappa_{D}}{\kappa_{A}} \sin (\theta) \mathrm{d} \theta
$$

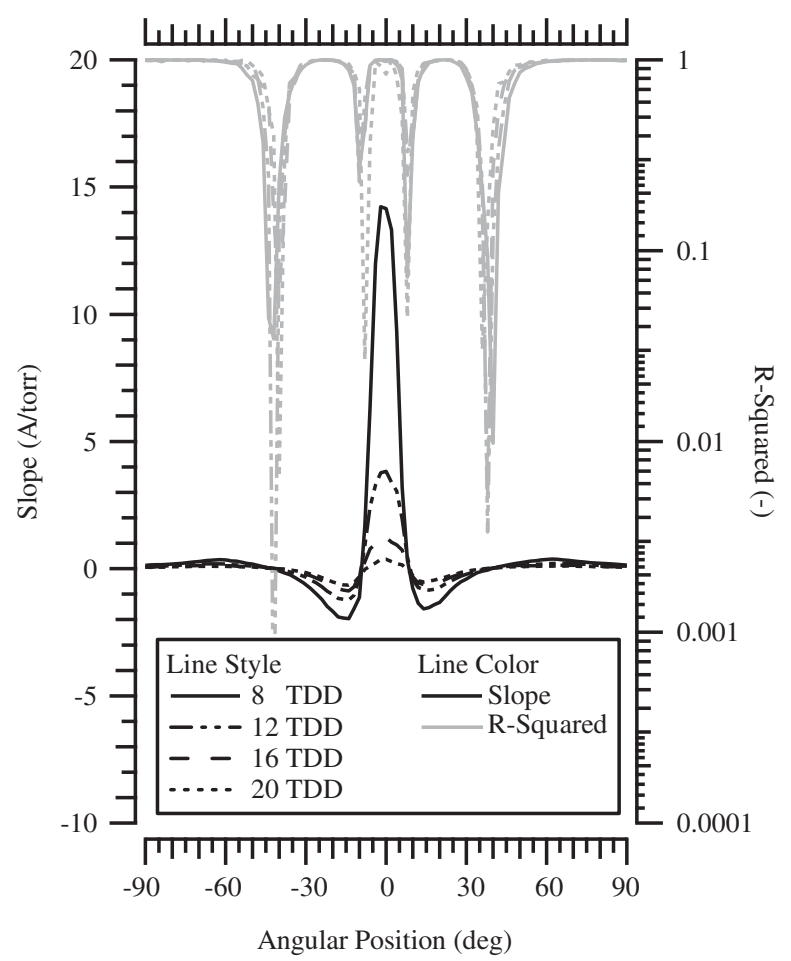

Fig. 19 Linear regression slope and coefficient of determination of ion current for a 200W HET.

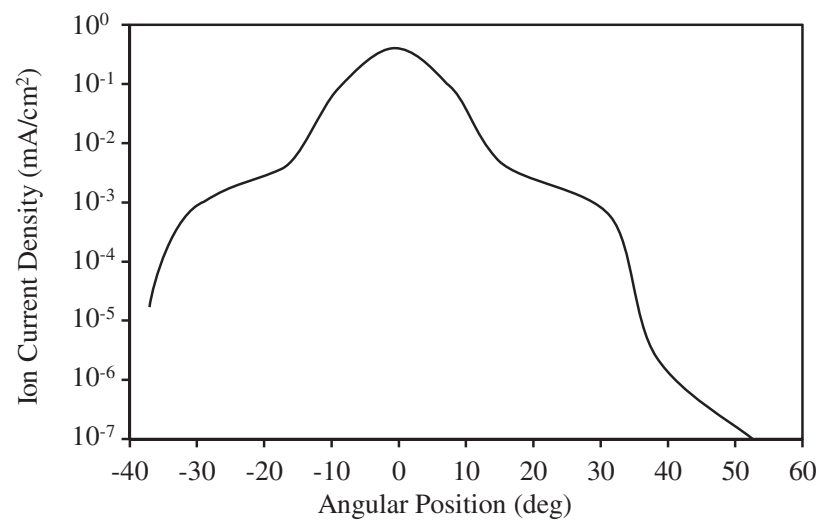

Fig. 20 Ion current density of the T5 GIT at $1.18 \mathrm{~m}$ downstream, recreated from [87]. 
Since Faraday probe plume measurements using hemispherical coordinates are often conducted from $\theta=0$ to $90 \mathrm{deg}$ on both the left and right sides of the plume in Fig. 7, the ion beam current in Eq. (8) may be calculated for each side of the plume and averaged. Two spatial correction factors, $\kappa_{D}$ and $\kappa_{A}$, are introduced to account for systematic error associated with the hemispherical coordinate system, where measurements are with respect to a point source as opposed to the thruster discharge geometry. The angular correction factor $\kappa_{A}$ and the distance correction factor $\kappa_{D}$ are formulated by approximating the thruster ions as originating from two point sources, as shown in Fig. 21. These point sources are located on the channel centerline at the exit plane of an annular HET in Fig. 21a. For GIT geometry, the point sources may be located at the midpoint between the GIT centerline and the outer diameter of the acceleration grid, as shown in Fig. 21b. Although the spatial correction factors $\kappa_{D}$ and $\kappa_{A}$ will not eliminate the systematic error introduced with the hemispherical coordinate system, the approximations serve to quantify the effect and reduce measurement error.

The correction factor $\kappa_{A}$ accounts for variations in probe angle with respect to the point sources, which affects the ion angle of incidence to the probe face and changes with angular position and distance. In addition, the ion angle of incidence is different for each point source. An average cosine loss in probe collection area is defined in Eq. (9), where the ion angles of incidence from the near- and far-point sources are calculated in Eq. (10) as $\alpha_{N}$ and $\alpha_{F}$, respectively. As shown in Fig. 21, the angle $\alpha_{N}$ refers to the ion angle of incidence from the near source and will alternate from the left to right sides of the diagram in

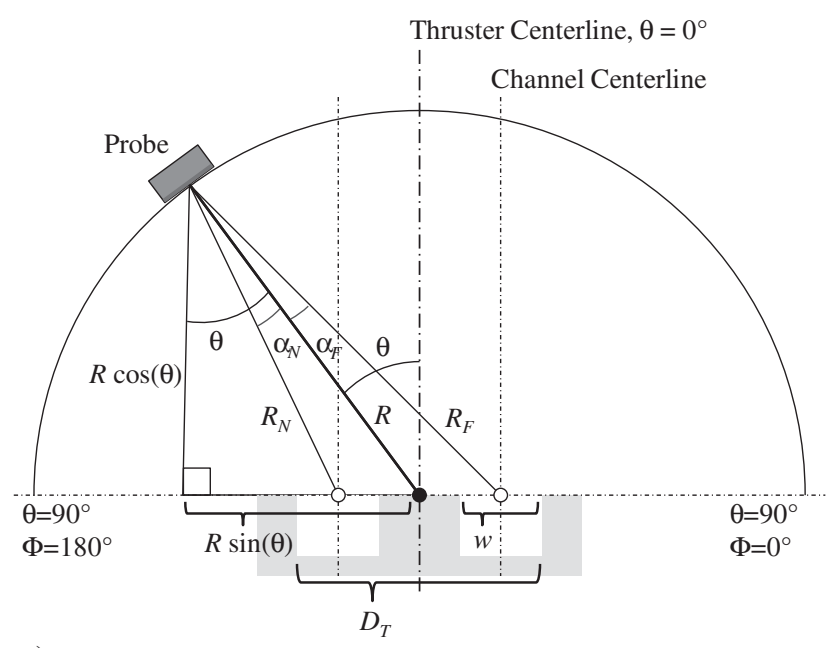

a)

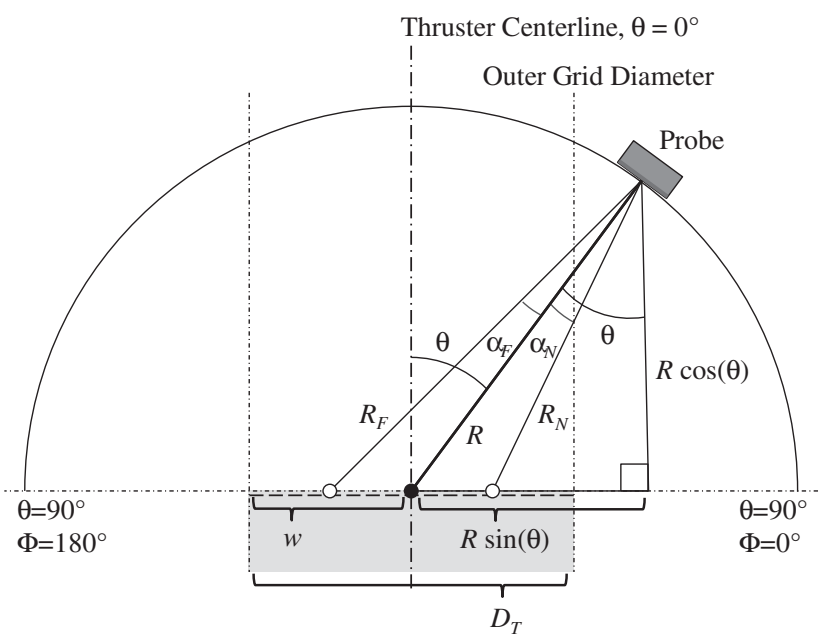

b)

Fig. 21 Measurement coordinate geometry showing probe distance and angular location in a two-point source model for a) annular HET geometry or b) GIT geometry.
Fig. 21 as the probe crosses thruster centerline. The width of the plasma discharge is denoted $w$, which is the width of the channel in an annular HET or $w=D_{T} / 2$ in a GIT:

$$
\begin{gathered}
\kappa_{A}=\cos \left(\frac{\alpha_{N}+\alpha_{F}}{2}\right) \\
\alpha_{N, F}\left[\theta, R, D_{T}\right]= \pm\left(\theta-\tan ^{-1}\left(\frac{\sin (\theta) \mp \frac{D_{T}-w}{2 R}}{\cos (\theta)}\right)\right)
\end{gathered}
$$

The correction $\kappa_{D}$ accounts for differences in path length from the near- and far-point sources to the probe, which would introduce a systematic error in the $R^{2}$ term in Eq. ( $\left.\underline{8}\right)$. Probe collector distances from the near- and far-point sources are characterized as $R_{N}$ and $R_{F}$, respectively. Similar to the analysis of ion angle of incidence, the path length will vary with probe angular position, and $R_{N}$ and $R_{F}$ will alternate from the left to right sides of the diagram in Fig. 21 as the probe crosses thruster centerline. The exception is on the thruster centerline, where the distance from the probe to each point source is equal and greater than $R$. Using the geometry in Fig. 21, $\kappa_{D}$ is defined in Eq. (11) based on the lengths $R_{N}$ and $R_{F}$, shown in Eq. (12):

$$
\kappa_{\mathrm{D}}=\left(\frac{1}{2}\left(\frac{R_{N}}{R}+\frac{R_{F}}{R}\right)\right)^{2}
$$

$$
\frac{R_{N, F}\left[\theta, R, D_{T}\right]}{R}=\sqrt{(\cos (\theta))^{2}+\left(\sin (\theta) \mp \frac{D_{T}-w}{2 R}\right)^{2}}
$$

The ratio of spatial correction factors $\left(\kappa_{D} / \kappa_{A}\right)$ is displayed for GITs $\left(w=0.5 D_{T}\right)$ and HETs $\left(w=0.15 D_{T}\right)$ as a function of probe angular position in Fig. 22 in terms TDD, calculated as $R / D_{T}$. The value of $w=0.15 D_{T}$ is a representative value for the HET channel width; however, a smaller channel will further increase the ratio $\left(\kappa_{D} / \kappa_{A}\right)$. The overall effect of $\kappa_{D} / \kappa_{A}$ is to increase current density in the plume central core, where ion current density is greatest, and thus the spatial corrections increase the calculated ion beam current in Eq. (8). The variation in $\kappa_{D} / \kappa_{A}$ decreases rapidly with downstream distance in the far-field, where the approximation of a point source measurement improves for hemispherical coordinate system. In Fig. 23, the correction on the thruster centerline shows the ratio of $\kappa_{D} / \kappa_{A}$ rapidly approaches unity with the downstream measurement distance, and it is less than 1.02 for distances greater than four TDD.

The spatial corrections are valid for beam ions originating near the exit plane. In addition, the correction only increases the ion source model from one to two, and it does not directly account for the accelerated ions across the full grid diameter or the channel width. This is expected to have a small effect on far-field measurements taken beyond four TDD, as evidenced by the comparison of $w=$ $0.15 D_{T}$ and $0.50 D_{T}$ in Fig. 23. Although the corrections are formulated assuming equal contribution of beam ions from each side of the channel or grid, this should also not be a significant source of error in the far-field plume.

The axial component of ion beam current is necessary for calculations of beam divergence, as discussed in Sec. V.E, and is formulated as

$$
I_{\text {Axial }}=2 \pi R^{2} \int_{0}^{\pi / 2} j[\theta] \frac{\kappa_{D}}{\kappa_{A}} \cos \left(\alpha_{A}\right) \sin (\theta) \mathrm{d} \theta
$$

where the angle $\alpha_{A}$ is introduced to calculate plume divergence with respect to the channel centerline in HETs or the grid radius midpoint in GITs as opposed to thruster centerline, as shown in Fig. 24 for HET geometry. Although this approach has not been applied to GIT plumes, the geometry will be equivalent to the grid radius midpoint of $w=0.5 D_{T}$. If the GIT outer grid diameter or the HET outer channel 


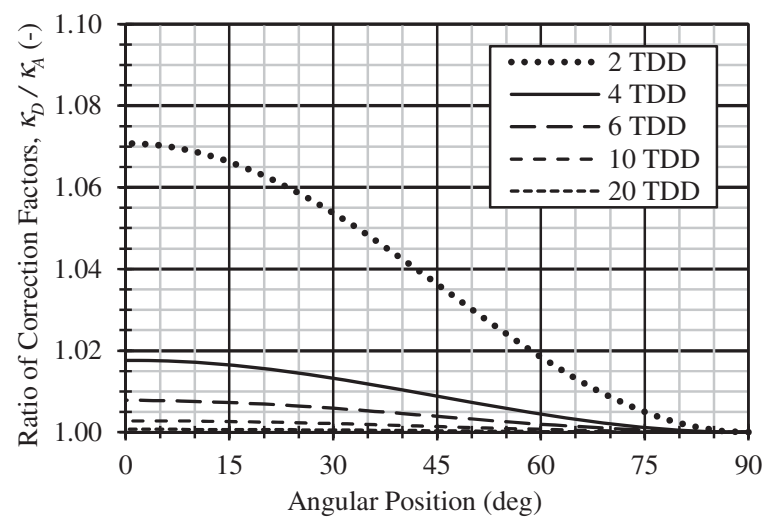

a)

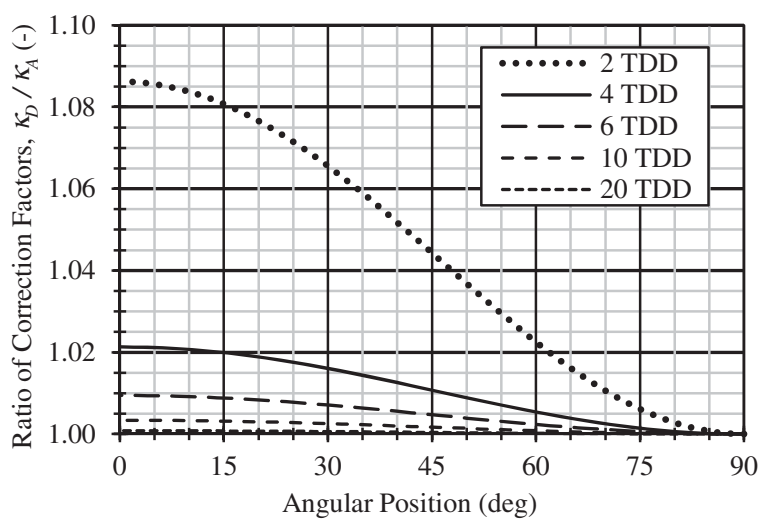

b)

Fig. 22 Combined correction factors $\left(\kappa_{D} / \kappa_{A}\right)$ for a) GIT with $w=0.5 D_{T}$ and b) HET with $w=0.15 D_{T}$.

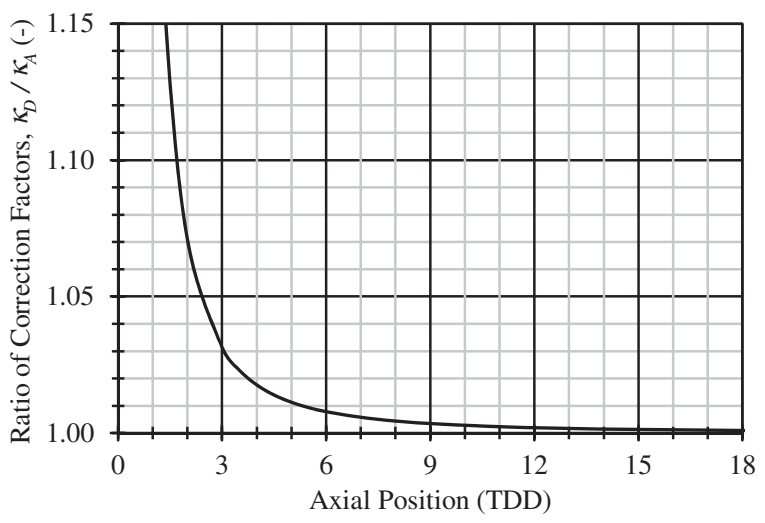

a)

Fig. 23 Combined correction factors $\left(\kappa_{D} / \kappa_{A}\right)$ on thruster centerline for a) GIT with $w=0.5 D_{T}$ and b) HET with $w=0.15 D_{T}$.

b) diameter is the preferred, then $w=0$. The parameter $\alpha_{A}$ is calculated as a piecewise function in Eq. (14):

$$
\begin{aligned}
& \alpha_{A}\left[\theta, R, D_{T}\right] \\
& =\left\{\begin{array}{cc}
\theta-\alpha_{N}=\tan ^{-1}\left(\frac{\sin (\theta) \mp \frac{D_{T}-w}{2 R}}{\cos (\theta)}\right) & \text { for } \sin ^{-1}\left(\frac{D_{T}-w}{2 R}\right) \leq \theta \leq 90 \mathrm{deg} \\
0 & \text { for } 0 \leq \theta \leq \sin ^{-1}\left(\frac{D_{T}-w}{2 R}\right)
\end{array}\right.
\end{aligned}
$$

Channel Centerline (HET) or Grid Radius Midpoint (GIT), $\theta=\sin ^{-1}\left(\left(D_{T}-w\right) / 2 R\right)$

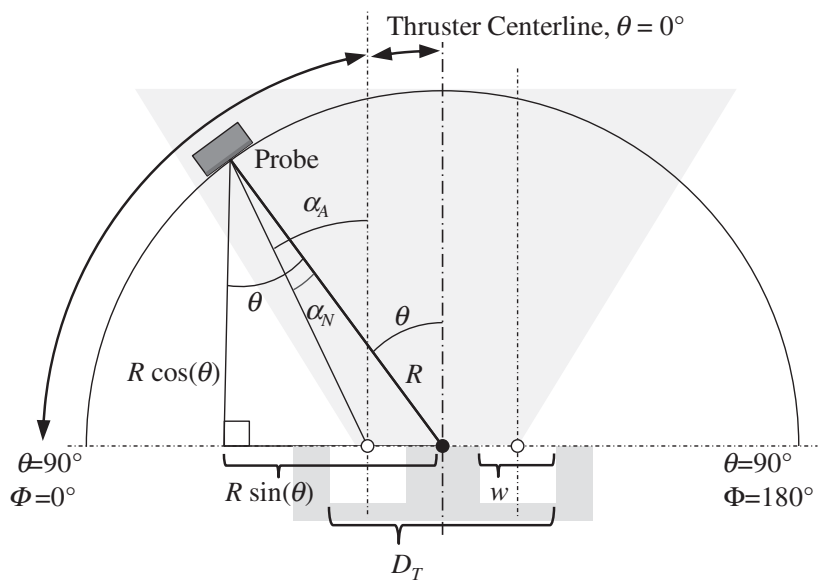

Fig. 24 Diagram of plume divergence with respect to channel centerline for a two-point source model.

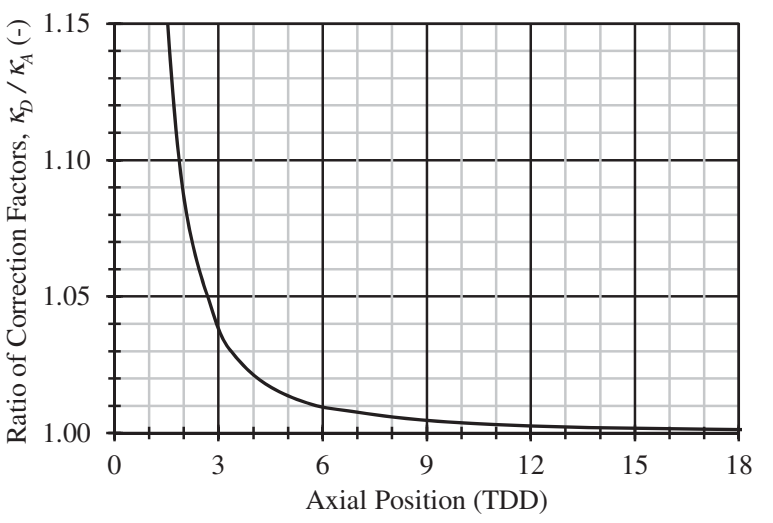

With the axial and total ion beam current, the charge flux weighted average $\operatorname{cosine}\langle\cos (\theta)\rangle_{j}$ is calculated to determine an effective farfield divergence angle $\lambda[\underline{88}]$.

$$
\left.\lambda=\cos ^{-1}(\langle\cos \theta)\rangle_{j}\right)=\cos ^{-1}\left(\frac{I_{\text {Axial }}}{I_{\text {Beam }}}\right)
$$

For Faraday probe plume measurements of the H6 HET in Figs. 16 and 17 , the variation in thruster discharge current $I_{d}, I_{\text {Beam }}$, and $I_{\text {Axial }}$ are shown in Fig. 25. The pressure characterizations indicate these plume properties increased by $10-20 \%$ compared to extrapolated vacuum conditions and, if unaccounted for, would represent a significant error in analysis of thruster divergence and utilization efficiencies. The linear increase in $I_{d}$ and $I_{\text {Axial }}$ with increasing background pressure is comparable in magnitude for all conditions, and it is consistent with increasing facility neutral ingestion, ionization, and acceleration. However, $I_{\text {Beam }}$ increased at a higher rate than thruster discharge current with increasing pressure, indicating $I_{\text {Beam }}$ was influenced by a facility interaction not related to the thruster discharge. The $I_{\text {Beam }}$ trend in Fig. 25 may be influenced by ionization of facility neutrals far downstream of the thruster discharge, which would not increase $I_{d}$. However, this is unlikely because the electron-neutral ionization MFP is greater than $10^{2} \mathrm{~m}$ (for $n>10^{18} \mathrm{~m}^{-3}, \sigma \sim 10^{-20} \mathrm{~m}^{2}$ ).

A more probable cause of the increased $I_{\text {Beam }}$ is associated with Faraday probe collection of the Bohm current on the plume periphery, described in Sec. IV.B. The Bohm current would have the greatest influence in regions of low beam ion current density, which is expected on the plume periphery. Although local plasma densities and electron temperatures in the H6 HET plume are not known for these Faraday probe measurements, plasma properties and variation with pressure may be estimated based on models of a $4.5-\mathrm{kW}$-class HET at $300 \mathrm{~V}$ discharge [89]. The model indicates local plasma conditions at $R=1 \mathrm{~m}$ (approximately six TDD in the H6 HET) are 


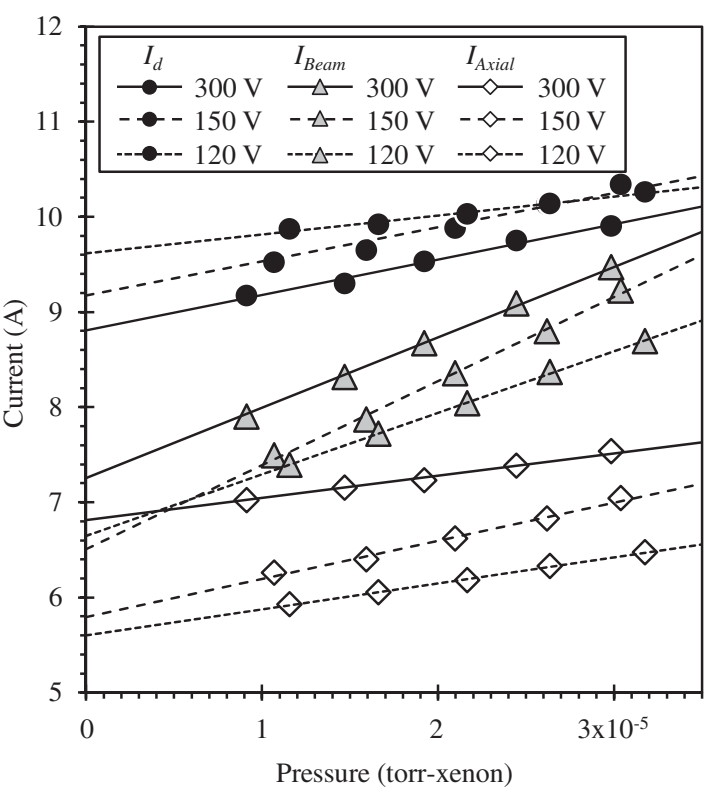

Fig. 25 Plume properties of the H6 HET at six TDD.

approximately 1.2 to $1.6 \mathrm{eV}$ and $10^{14}$ to $10^{16} \mathrm{~m}^{-3}$ on the plume periphery over a range of high background pressure conditions comparable to the H6 HET measurements in Figs. 16 and 17. Based on Eq. (3), $j_{\text {Bohm }}$ would be $0.001-0.1 \mathrm{~mA} / \mathrm{cm}^{2}$, which may be a significant fraction of the measured ion current density on the plume periphery beyond approximately $\pm 30 \mathrm{deg}$ from centerline in Fig. 16. Confirming this cause for increased $I_{\text {Beam }}$ with increasing background pressure requires experimental data, such as local Langmuir probe measurements of plasma density and electron temperature to subtract $j_{\text {Bohm }}$ from Faraday probe measurements. These trends are further examined in Sec. V.E with respect to the beam divergence and thruster utilization efficiencies.

\section{Near-Field Hall Thruster Plume}

A systematic investigation of facility interactions on the near-field HET ion current density has not been conducted, though there are several Faraday probe studies on the spatial variation of ion current density in this region for multiple HET technologies [7, 41, 44,45,90]. Characterization of the near-field HET plume at multiple background pressures is recommended to enable extrapolation of ion current density to zero pressure, similar to the linear regression of far-field ion current density in Sec. V.B.

The near-field ion current density measurements from four HET designs are shown in Fig. 26, with thruster operation ranging from 2 to $50 \mathrm{~kW}$. This includes the NASA-457Mv2 at $500 \mathrm{~V} / 50 \mathrm{~kW}$ [54] in Fig. 26a, the NASA-300M at $500 \mathrm{~V} / 20 \mathrm{~kW}$ [54] in Fig. 26b, the H6

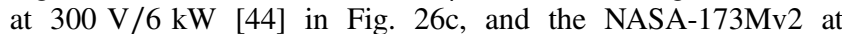
$500 \mathrm{~V} / 2.2 \mathrm{~kW}[4 \overline{1}]$ in Fig. 26d. These near-field measurements illustrate features common to the annular HET designs, and they range from less than 0.1 TDD to beyond two TDD. For all four thruster designs, the plume merges at approximately 0.5-1.0 TDD. Downstream of the transitional region of plume merging, the plume interactions are complex and complicate data analysis. Negative collected current near the thruster was observed in Fig. 26d near $100 \mathrm{~mm}$ radial distance and $25 \mathrm{~mm}$ downstream of the exit. The negative current was attributed to a region of reduced plasma potential where the electrons were $5-10 \mathrm{eV}$; thus, the probe bias potential was insufficient to achieve ion saturation [41]. Although a more negative bias may mitigate the effect, it may also affect probe sheath expansion and induce thruster perturbations. These issues were minimal at greater than 0.2 TDD in Figs. $26 \mathrm{~b}$ and $26 \mathrm{~d}$. In these studies, the recommended near-field measurement range would extend from 0.2 to 0.5 TDD downstream of the thruster exit. Nearfield HET plume measurements outside of this range may be desirable for model validation and investigation of ion acceleration processes or the transition region; however, the probe perturbations, sheath effects, and plume merging effects should be addressed.

A common HET feature is the region of high ion current density on thruster centerline, which is often visually observed and described as the "center spike" or "dovetail" [72]. This is evident in Fig. 26 for all thrusters upstream of the region of beam merging, including those with a centrally mounted cathode or external cathode configuration. The center spike contains beam ions, whereas the cathode plume does not. As stated in Sec. III.B, in the case of an externally mounted cathode, the recommended approach is to sweep the Faraday probe in a plane orthogonal to the plane containing the cathode. In the case of a centrally mounted cathode, the recommended approach is to remove the contributions of the cathode plume from the Faraday probe results, as shown in Appendix B.

The total ion beam current from near-field plume measurements of ion current density is calculated with Eq. (5) for a fixed downstream distance $Z$, written as

$$
I_{\text {Beam }}=2 \pi \int_{0}^{\infty} j[r] r \mathrm{~d} r
$$

Note the measured ion current density $j[r]$ in near-field measurements is simply the axial component of current density; therefore, $I_{\text {Beam }}=I_{\text {Axial }}$ as the radial integration limit approaches infinity. In contrast to Eq. (8) used for the hemispherical coordinate system, no correction factors associated with coordinate system geometry are necessary in Eq. (16). Since near-field Faraday probe measurements are typically conducted across the entire thruster, the ion beam current in Eq. (16) may be calculated for each side of the plume and averaged. The radial integration in Eq. (16) is limited in practice by low signal strength and probe proximity to the facility walls. A $0.2 \%$ threshold-based integration limit may be used, as described in Sec. IV.D. The integrated ion beam current is sensitive to integration limit; therefore, it is recommended to characterize $I_{\text {Beam }}$ as a function of the integration limit [45] and correlate the results to far-field Faraday probe results or assess with a thruster efficiency analysis $[45,88]$, if resources allow. The recommended radial span of $1.5 \bar{D}_{T}$ from thruster centerline was sufficient for the $0.2 \%$ threshold-based limit from 0.2 to 0.5 TDD in Fig. 26b.

Evaluation of HET beam divergence from near-field Faraday probe data is complicated by the proximity to the thruster exit plane, since the ion current point of origin cannot be assumed at the channel midpoint or outer channel diameter, nor emanating from the exit plane. Although the bulk HET plasma is directed along the firing axis, the local plasma jet from the annular channel may be traveling toward or away from the thruster centerline. The iterative path-finding method may be used to determine the effective origin of the plasma plume within the channel, and thereby used as a reference for ion beam divergence. The points of origin from each side of the HET channel are illustrated as a two-dimensional channel cross section in Fig. 27, where the variables $\theta_{j}$ and $\delta_{j}$ are the travel angle and divergence angle of the jet, and $r_{0}$ and $z_{0}$ are the radial and axial coordinates of the jet point of origin, respectively.

The iterative path-finding method starts with the assumption that the near-field annular HET plasma plume can be treated as a freeexpanding jet, such that the travel and divergence angles do not change as a function of axial distance downstream. Based on past experiments, this assumption was only valid downstream of the evolving jet structure (greater than 0.2 TDD) and the region upstream of merging beams from opposite sides of the annular channel (less than 0.5 TDD) [45].

The iterative path-finding method iterates between two calculation steps. In the first step, an initial estimate of the point of origin is applied to expressions of the travel angle in Eq. (17) and the divergence angle in Eq. (18):

$$
\tan \left(\theta_{j}\right)=\frac{2 \pi \int_{0}^{\infty} j[r, z] \frac{r-r_{0}}{z-z_{0}} r \mathrm{~d} r}{2 \pi \int_{0}^{\infty} j[r, z] r \mathrm{~d} r}
$$




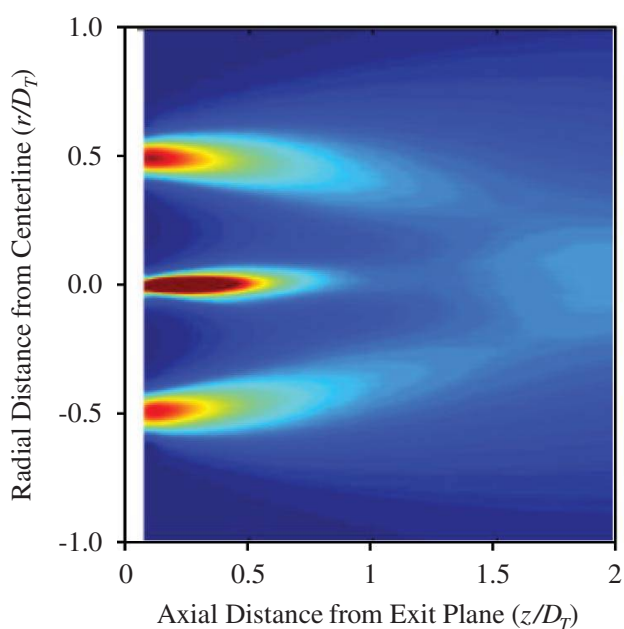

a)

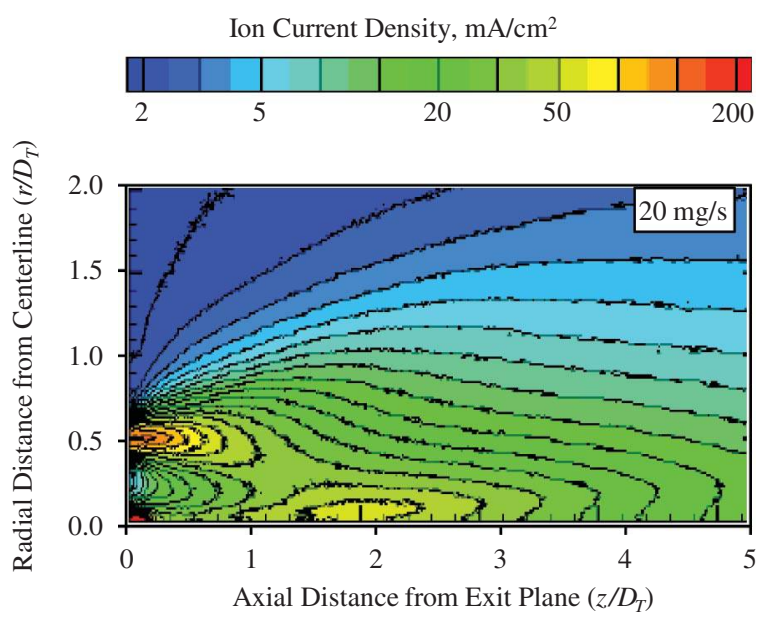

c)

Fig. 26 Ion current density measurements of the a) NASA-457Mv2 [54

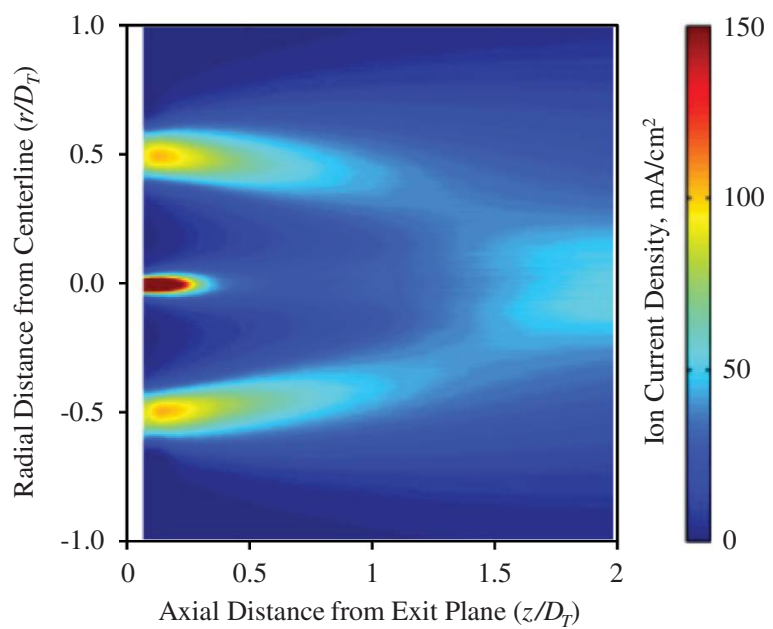

b)

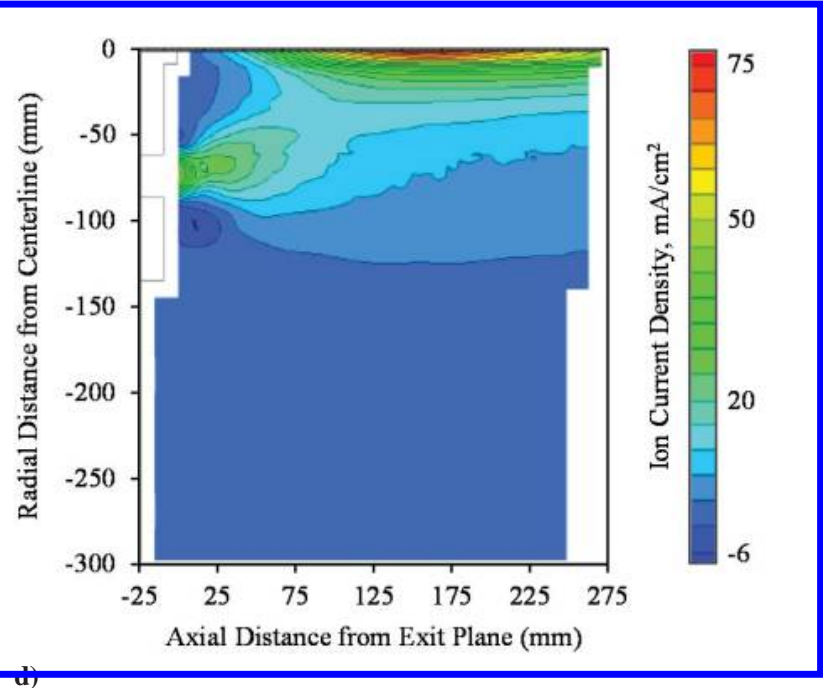

In contrast to the expression for ion beam current in Eq. (16), the iterative path-finding method in Eqs. (17) and (18) is generally insensitive to changes in the choice of integration limit.

In the second step, variations in the travel and divergence angles with axial distance are used to compute the error metrics and update the point of origin location on each side of the plume. Equations (19) and (20) are feedback equations that are used to update the point of origin and may be reinserted into Eqs. (17) and (18). Equations (21) and (22) are the error metrics for radial and axial coordinates, respectively:

$$
\begin{gathered}
r_{0, m+1}=r_{0, m}-\beta_{1} \sin \left(\frac{r_{0, m-1}-r_{0, m}}{E_{\theta, m-1}-E_{\theta, m}}\right) E_{\theta, m} \\
z_{0, m+1}=z_{0, m}-\beta_{2} \sin \left(\frac{z_{0, m-1}-z_{0, m}}{E_{\delta, m-1}-E_{\delta, m}}\right) E_{\delta, m} \\
E_{\theta}=\left[\frac{1}{N} \sum\left(\theta_{j}-\bar{\theta}_{j}\right)^{4}\right]^{1 / 4} \\
E_{\delta}=\left[\frac{1}{N} \sum\left(\delta_{j}-\bar{\delta}_{j}\right)^{4}\right]^{1 / 4}
\end{gathered}
$$


where the subscript $m$ indicates the $m$ th iteration, $\beta_{1}$ and $\beta_{2}$ are tuning parameters to control the speed of convergence, $E_{\theta, m}$ is the error metric of the travel angle, $E_{\delta, m}$ is the error metric of the divergence angle, $\bar{\theta}_{j}$ is the mean travel angle, $\bar{\delta}_{p j}$ is the mean divergence angle, and $N$ is the number of axial positions over which the angles were calculated. This process is typically repeated on both sides of the measurement sweep, for $r$ greater than zero and $r$ less than zero.

Convergence of the iterative path-finding method for the point of origin within the channel is shown for the NASA-300M operating at $500 \mathrm{~V}, 20 \mathrm{~kW}$ in Fig. 28 [45], where the black lines represent the origin for the jet in the channel for $r$ greater than zero and the red lines represent the opposite channel where $r$ is less than zero [45]. The corresponding integrated total ion beam current, travel angles, and divergence angles are shown in Fig. 29, where dashed vertical lines indicate the boundaries of the jet analysis zone for near-field plume [45]. Although this dataset extends beyond the defined near-field plume, the expansive range shows the variation in parameters outside of the 0.2-0.5 TDD region. The trends were consistent for nearfield Faraday probe measurements over a wide range of operating conditions for the both NASA-300M and NASA-457Mv2 HETs [45].

To calculate the overall beam divergence angle as a global plume parameter of annular HETs, the jet momentum is assumed to be decomposed into the momenta of two collimated beams from the channel. The beams each carry half of the momentum of the associated jet and radiate outward from the jet travel angle at plus/ minus the jet divergence angle, as shown in Fig. 27. This approach is represented mathematically in Eq. (23):

$$
\delta=\frac{\left|\theta_{j}+\delta_{j}\right|+\left|\theta_{j}-\delta_{j}\right|}{2}
$$

For Faraday probe sweeps across the channel, the divergence angle should be averaged from each side of the thruster, where $r$ is greater than zero and $r$ is less than zero. The near-field HET divergence halfangle $\delta$ is suitable for evaluation of thrust loss, and ideally is equivalent to the effective far-field divergence angle $\lambda$. The HET beam divergence using the iterative path-finding method was compared to evaluations of the outer beam threshold limit and to far-field Faraday probe measurements [45,54]. The iterative path-finding method generally provided good agreement with farfield measurements and expected divergence based on thruster performance, whereas the outer beam threshold limits consistently underpredicted divergence.

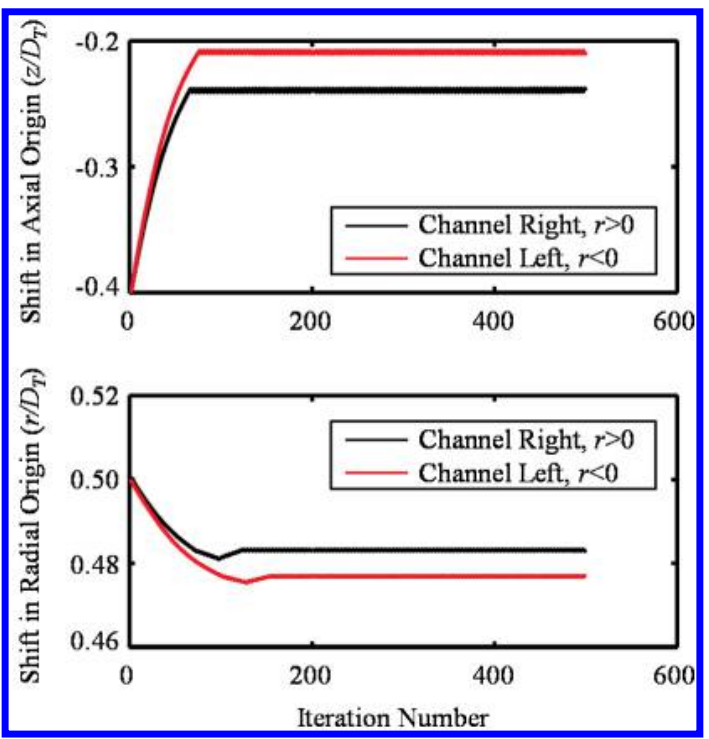

Fig. 28 Convergence of the iterative path-finding method of the NASA$300 \mathrm{M}$ plume [45].

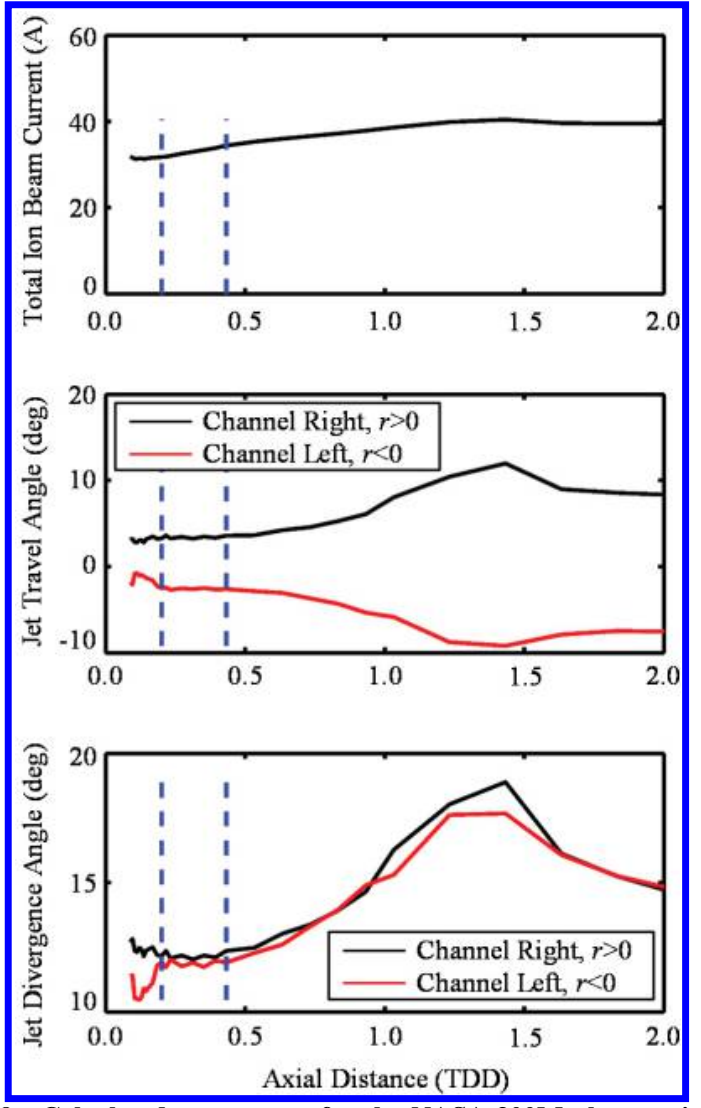

Fig. 29 Calculated parameters for the NASA-300M plume using the iterative path-finding method [ㅍ].

\section{Near-Field Gridded Ion Thruster Plume}

As with the near-field HET plume, a systematic investigation of facility interactions on the near-field GIT ion current density has not been conducted. Facility effects associated with background pressure, such as CEX collisions and propellant ingestion, on the GIT plume may be characterized similar to far-field characterizations. However, there are unique considerations for GITs. Near-field test recommendations described in Secs. IV.D and IV.E are expected to provide the necessary data to predict time-averaged ion current density properties in the space vacuum environment.

In the near-field GIT plume, a Faraday probe can be used to evaluate ion plume properties, infer plasma uniformity, and resolve features of the ion beamlet structure in the very near-field. Ion plume profiles may be integrated according to Eq. (16) to estimate the actual extracted beam current and compare to the beam current measured by the ion optics. In many cases, the integrated ion beam current measured by Faraday probes is greater than $10 \%$ larger than measured by ion optics [61,91-93], which may be associated with not using corrections described in Sec. V.A, such as $\kappa_{G}$ for the effective probe collection area. However, the integration is also complicated by asymmetry in the near-field ion current density beam profile, specifically near the thruster centerline axis [94]. The location of peak ion current density is often specific to a particular engine. The physical origin of this effect is not well understood, although it has been speculated to be associated with peculiarities in cathode operation and coupling to the anode. Figure 30 illustrates typical ion current measurements of a nude Faraday probe ranging from approximately 0.16 to $1.7 \mathrm{TDD}(\sim 5$ to $50 \mathrm{~cm})$ downstream of the ion optics of an NSTAR thruster at $1.76 \mathrm{~A}$ beam current and $2.3 \mathrm{~kW}$ input power $[92,95]$. In these measurements, the peak ion current density profile is left of the thrust axis, and the left and right plume profiles show slight asymmetry about the peak. Similar behaviors were observed in the NEXT near-field ion current density profiles [21,93]. In cases of plume asymmetry, past studies have calculated differences in integrated ion beam current exceeding $20 \%$ on the left or right side of the plume [92]. To mitigate the asymmetry, the ion current density 


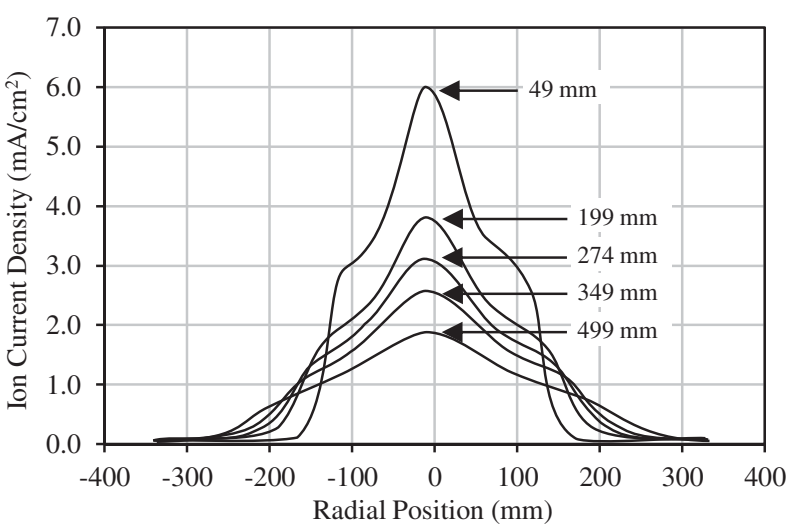

Fig. 30 Ion current density profiles of the $30 \mathrm{~cm}$ NSTAR thruster, recreated from [92].

profile was shifted radially to align the peak to thruster centerline. The plume divergence and ion beam current were then averaged from each side of the redefined profile $[61,92]$. This approach can lead to large error, and it should be included in error analysis if it is implemented.

Similar to Faraday probe measurements of the near-field HET plume in Sec. V.C, the radial extent of integration limits for GIT plumes using Eq. (16) is uncertain. Additional studies are necessary to determine the suitable limits of integration. However, since the GIT beam current is known based on ion optics, the integrated ion beam current from near-field plume measurements is often scaled and, in many cases, the radial measurement range is limited to the central beam. Traditionally, the primary use of Faraday probe measurements of GIT plumes has been to determine the plume divergence for calculations of thrust. To this end, near-field Faraday probe measurements at fixed axial location are used to calculate the fraction of total ion beam current enclosed within a given radius, shown in Eq. (24) for the $95 \%$ fraction:

$$
0.95 I_{\text {Beam }}=2 \pi \int_{0}^{r_{95}} j[r] r \mathrm{~d} r
$$

Note that the value of $I_{\text {Beam }}$ in Eq. (24) is typically the beam current measured by ion optics, but it could also be the integrated ion beam current from Faraday probe measurements. If the integrated ion beam current is used, the radial integration limits will impact the radius enclosing a given fraction of the beam.

In Fig. 31, the enclosed current fractions from 20 to $99 \%$ are shown for the ion current density profiles in Fig. 30, where $I_{\text {Beam }}$ is determined by GIT ion optics. This GIT dataset reveals the spatial distribution of calculated enclosed current fractions yields a linear relationship in the near-field plume, where the arctangent of the

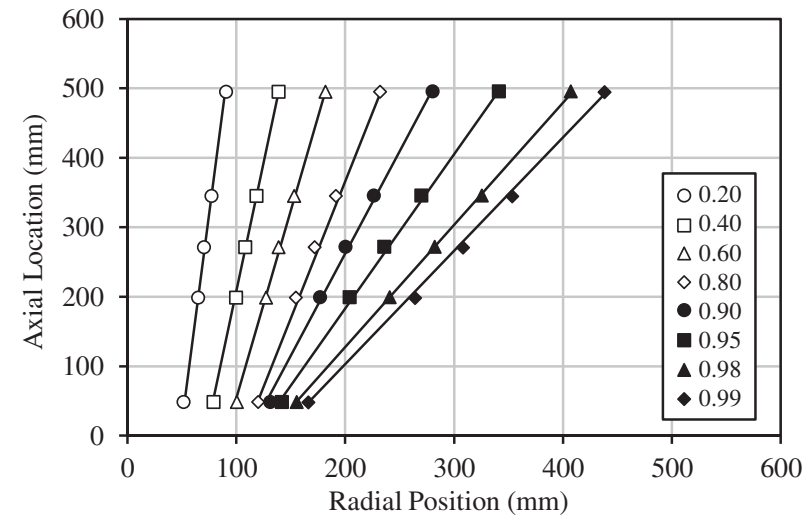

Fig. 31 Fractions of total integrated beam current of the $30 \mathrm{~cm}$ NSTAR thruster, recreated from [92]. best-fit linear regression slope $S_{f}$ of each enclosed current fraction may be used to determine an effective divergence half-angle $\beta$ of the GIT near-field plume as defined in Eq. (25):

$$
\beta=\tan ^{-1}\left(\frac{1}{S_{f}}\right)
$$

For this method to be meaningful, the Faraday probe radial measurement sweeps must extend beyond the beam central core and multiple axial distances are required. Figure 32 shows is the enclosed current fractions from Fig. 31 relative to thruster beam current and corresponding $\beta$. The relevant enclosed current fractions used for evaluation of thrust loss parameter are often 90,95 , or $99 \%$. Ideally, the near-field GIT divergence $\beta$ should be equal to the effective farfield divergence half-angle $\lambda$ if facility pressure interactions are suitably isolated and mitigated in data analysis.

This approach was used for the $30 \mathrm{~cm}$ GITs on the Dawn ion propulsion system and demonstrated good agreement between flight thrust levels and predictions using the $90 \%$ beam divergence halfangles from ground measurements [75]. Nevertheless, the method requires further analysis and the assumptions should be evaluated for future systems. For example, it is not clear that the $90 \%$ enclosed current fraction is the most suitable nor whether the integrated total ion beam current should be used instead of the beam current from ion optics for this calculation. In addition, the results may benefit from plume characterization at multiple background pressures to improve consistency between the total ion beam current calculated with Faraday probe plume measurements and the values determined from the ion optics. Unlike far-field Faraday probe measurements, Faraday probe measurements of the near-field GIT plume are not significantly affected by CEX collisions due to the large mean free path relative to the plume region of interest. Instead, background pressure characterizations of the near-field GIT plume may enable evaluation of processes associated with facility neutral ingestion and features associated with the beamlets. For example, Faraday probe studies of the very near-field plume region of the NEXT indicated beamlet focusing was a better indicator of far-field GIT beam divergence than the near-field region downstream of beamlet merging. Alternative approaches should also be considered, such as the iterative pathfinding method described for near-field HET plumes in Sec. V.D. Thus, a systematic evaluation of background pressure effects on the GIT near-field plume and the advantages, or limitations, of the different analysis techniques should be conducted to establish standard test practices for Faraday probe measurements of near-field GIT plumes.

\section{E. Global Plume Properties}

Thruster plume properties calculated with Faraday probe measurements may be used to quantify thruster performance loss mechanisms and determine utilization efficiencies. The development and derivation of these parameters may be found elsewhere in the literature $[\underline{6}, \underline{88}]$.

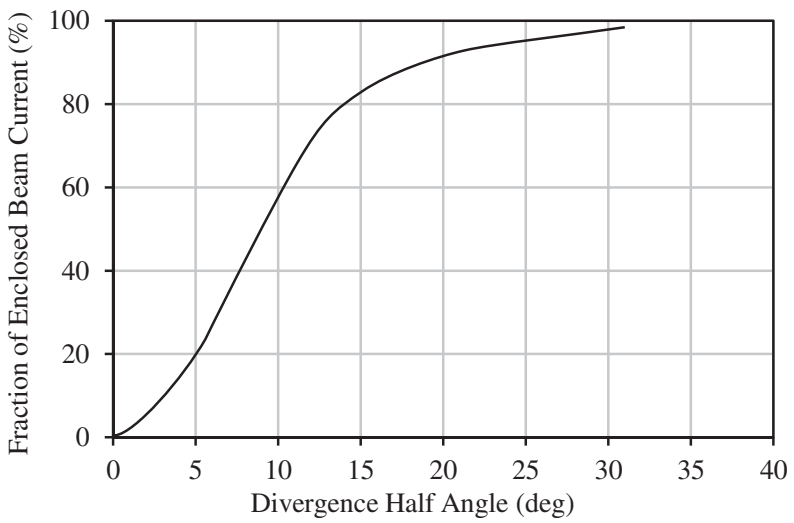

Fig. 32 Fraction of enclosed ion beam current and corresponding divergence half-angle of the $30 \mathrm{~cm}$ NSTAR thruster, recreated from [ $\underline{92}$ ]. 
Current utilization is defined by the ratio of ion beam current to input discharge current as

$$
\eta_{\text {Current }}=\frac{I_{\text {Beam }}}{I_{d}}
$$

The thruster mass utilization may be calculated with Eq. (27) as the ratio of the ion mass flow rate to the total thruster mass flow rate, which includes both anode and cathode propellant flows. The ion mass flow rate is calculated using ion beam current and the average ion charge $Q$ from measurements of ion species charge fractions in the plume in Eq. (28):

$$
\eta_{\mathrm{Mass}}=\frac{I_{\mathrm{Beam}}}{\dot{m}_{T} Q} \frac{M_{i}}{e}
$$

where

$$
Q=\left(\sum_{k=1} \frac{\Omega_{k}}{Z_{k}}\right)^{-1}
$$

The beam utilization, also known as divergence utilization, is a measure of momentum losses in axial thrust due to plume divergence. Losses in directed thrust may be estimated using divergence of ion beam current. A past analysis indicated the differences in divergence in momentum and divergence of ion current are primarily affected by spatial variation in ion charge species fractions within the central core of the plume [88]. Variation in the ion charge state within the ion beam is small and, typically, $Q$ may vary less than $5 \%$ [96]; thus, the assumption that divergence in ion current is representative of divergence in momentum is suitable. The beam utilization may be expressed in Eq. (29) with $\lambda$ for far-field plume measurements. Replacing divergence half-angle $\lambda$ with $\delta$ or $\beta$ enables calculation of beam utilization for near-field HET measurements or near-field GIT measurements, respectively:

$$
\eta_{\text {Beam }}=\cos ^{2}(\lambda)=\sqrt{F_{t}}
$$

The thrust loss parameter $F_{t}$ in Eq. (29) is often used to calculate thrust in Eq. (30), where $\alpha$ represents thrust reduction due to multiply charged ions, $M_{i}$ is the ion mass, and $V_{\text {Beam }}$ is the average ion acceleration voltage [6]. In practice, the uncertainty in measured global plume parameters has higher uncertainty than direct measurements of thrust. This approach is used more often for GITs, since $I_{\text {Beam }}$ and $V_{\text {Beam }}$ are known from the ion optics:

$$
T=\alpha F_{t} I_{\text {Beam }} \sqrt{\frac{2 M_{i} V_{\text {Beam }}}{e}}
$$

Calculated values of $\eta_{\text {Current }}, \eta_{\text {Mass }}$, and $\eta_{\text {Beam }}$ are shown in Fig. 33 for H6 HET operation at $10 \mathrm{mg} / \mathrm{s}$ anode flow and $7 \%$ cathode flow, based on plume properties in Fig. 25. The mass utilization was calculated with Eq. (27), using measurements of ion species current fractions measured at $1 \mathrm{~m}$ on the H6 HET channel centerline [88]. The trends in Fig. 33 over the range of $1 \times 10^{-5}$ to $3 \times 10^{-5}$ torr-Xe were primarily associated with increased $I_{\text {Beam }}$ in Fig. 25 , and they revealed $\eta_{\text {Current }}$ increased by $0.1, \eta_{\text {Mass }}$ increased by $0.16-0.21$, and $\eta_{\text {Beam }}$ decreased by $0.09-0.16$. The extrapolations of $\eta_{\text {Current }}, \eta_{\text {Mass }}$, and $\eta_{\text {Beam }}$ to zero pressure provided $R$-squared greater than 0.99 for all operating conditions. In addition, these values at zero pressure were consistent with analysis of H6 HET utilization efficiencies in [88], which were calculated with a combination of ion energy diagnostics in the plume and performance measurements. Thus, the extrapolation of Faraday probe ion current density to zero pressure may be partially mitigating the effect of the Bohm current, and this increases confidence in the approach to estimate global properties in the space environment.

The global far-field plume properties of a 200 W HET with variation in facility pressure and downstream distance are shown in Figs. 34-36, based on plume measurements in Figs. 18 and $\underline{19}$. This unique dataset

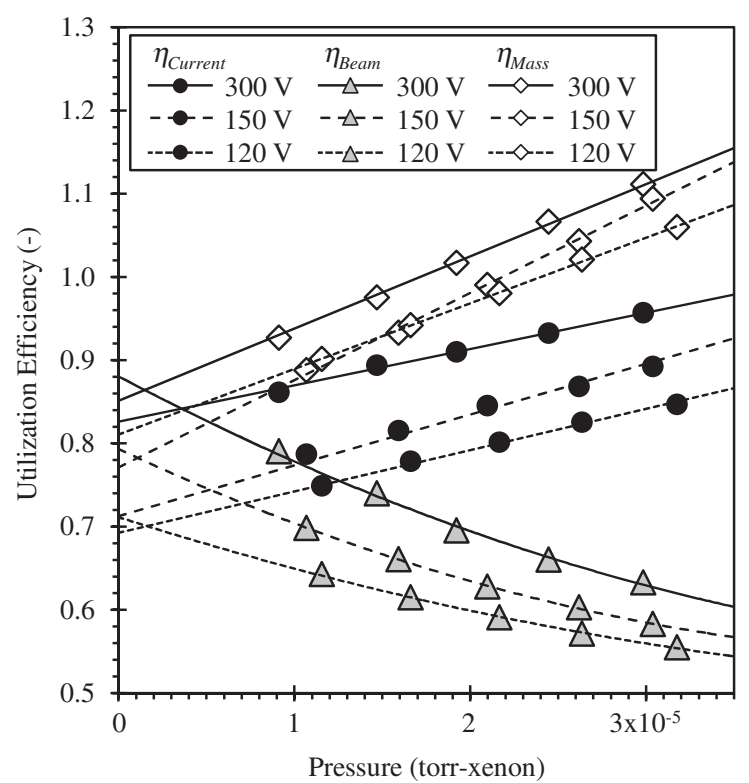

Fig. 33 Current utilization, beam utilization, and mass utilization of the H6 HET at six TDD.

included all corrections in Secs. V.A and V.B, and it was conducted with a nested Faraday probe that enabled simultaneous measurements of the HET plume with four probe collector and guard ring geometries [18]. The precision in measured ion current density profiles, calculated

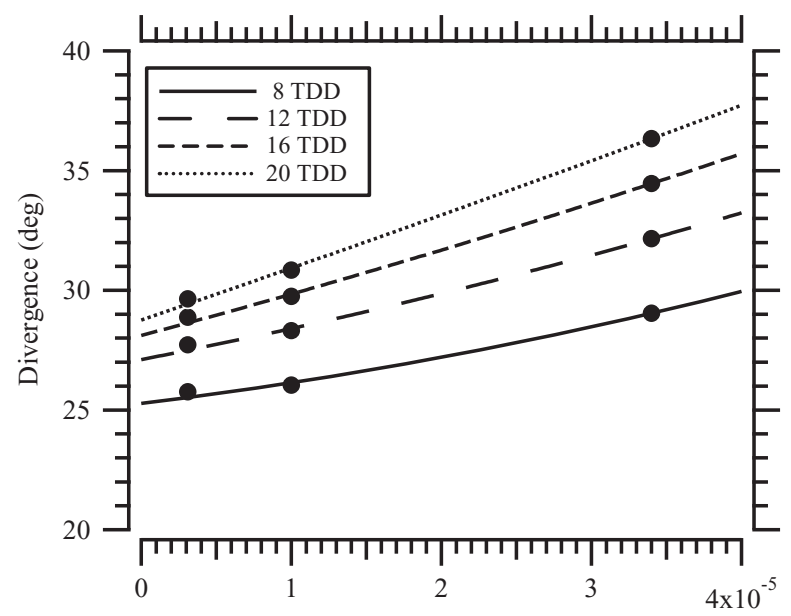

a)

$$
\text { Pressure (torr-xenon) }
$$

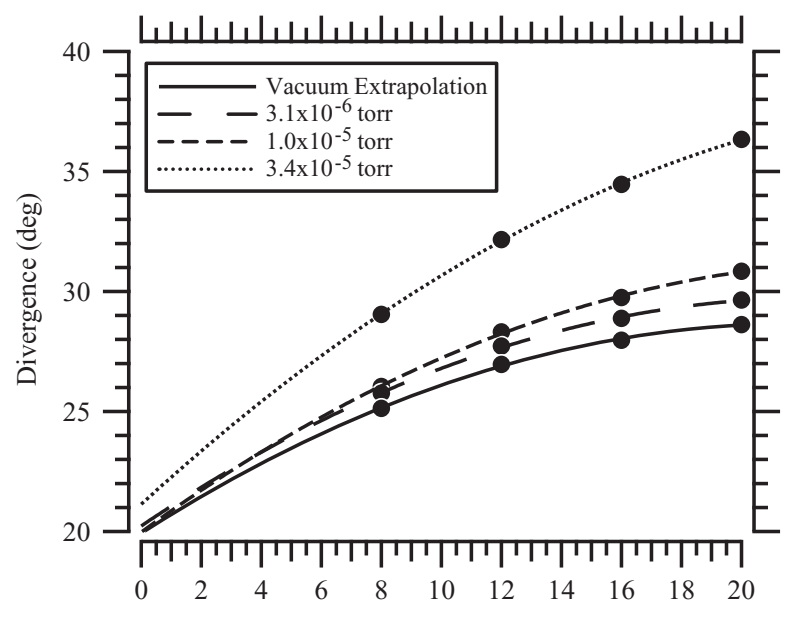

b)

Axial Distance (TDD)

Fig. 34 Beam divergence of a $200 \mathrm{~W}$ HET as a function of a) facility pressure and b) measurement distance. 
global plume properties, and extrapolations to zero pressure between four probe geometries and over a wide range of background pressure and regions of the far-field plume in [18] increased confidence in the results and test methodologies. No such dataset is known for GITs or near-field HET plumes.

The data in Figs. 34-36 reveal several key considerations for Faraday probe measurements of a far-field HET plume. First, the variations in global plume properties with pressure show the impact of facility background particles are nonnegligible at all far-field distances and angular locations. Second, the extrapolations to zero pressure in Figs. 34a, 35a, and 36a yielded different results at fixed $R$. These trends suggest a natural evolution of the plume unrelated to facility effects, which may be associated with HET plume merging or CEX collisional processes between beam ions and neutral propellant from the thruster and cathode. This outcome is in agreement with results in Figs. 34b, $35 \mathrm{~b}$, and $36 \mathrm{~b}$, where the global plume properties (at fixed pressure) extrapolated to the thruster exit plane at zero TDD yielded consistent values of $\lambda, \eta_{\text {Current }}$, and $\eta_{\text {Beam }}$; this was true for the three background pressures characterized and the values extrapolated to zero pressure.

The reduction in $\eta_{\text {Beam }}$ with increasing pressure for the $200 \mathrm{~W}$ HET in Fig. 35a was associated with ion scattering, and it was consistent with the reduction shown for the H6 HET in Fig. 33, and it was associated with ion scattering. However, the extrapolation of $200 \mathrm{~W}$ HET $\eta_{\text {Beam }}$ to zero TDD in Fig. 35b reveals the beam utilization increases near the thruster exit plane, ranging from 0.81 at eight TDD to 0.89 at zero TDD for the lowest pressure condition $3.1 \times 10^{-6}$ torr-Xe. The value $\eta_{\text {Beam }}$ extrapolated to zero TDD ranged from 0.88 to 0.89 for all facility pressure conditions in Fig. $35 \mathrm{a}$, and the corresponding divergence half-angle $\lambda$ at zero TDD ranged from 20 to $21 \mathrm{deg}$ for
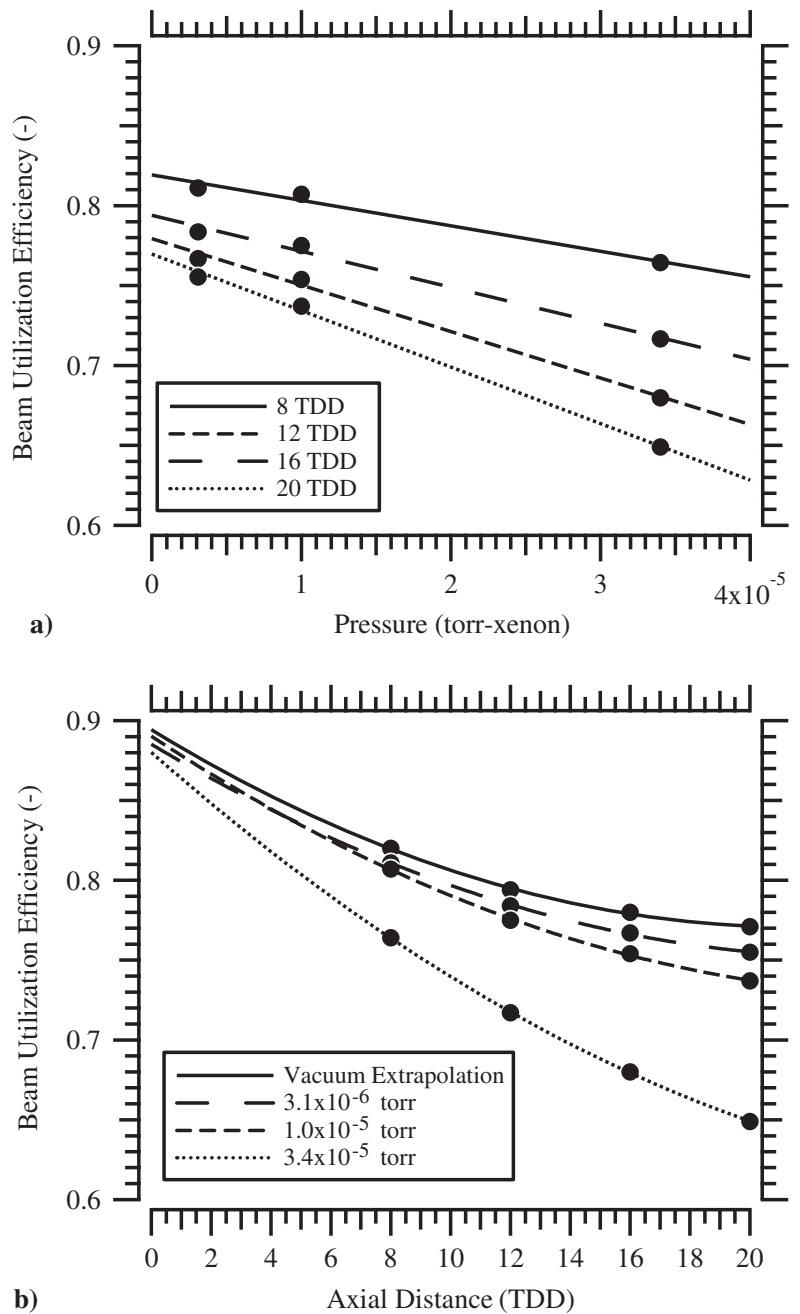

Fig. 35 Beam utilization efficiency of a $200 \mathrm{~W}$ HET as a function of a) facility pressure and b) measurement distance. all facility pressure conditions in Fig. 34a. The consistent estimates of $\eta_{\text {Beam }}$ and $\lambda$ at zero TDD from Faraday probe measurements demonstrate the value of characterizing the HET plume across a wide range of facility pressures and measurement distances. Based on this result, the values of $\eta_{\text {Beam }}$ for H6 HET in Fig. 33 at a single distance are likely underestimating the true plume divergence as a thruster performance loss mechanism [88].

The reduction in $200 \mathrm{~W} \mathrm{HET} \eta_{\text {Current }}$ with increasing pressure in Fig. 36a is opposite to the H6 trends in Fig. 33. However, the $200 \mathrm{~W}$ HET trend has been observed in other thrusters [97], and different HET behaviors have been observed with increasing facility pressure in multiple past studies [73,98,99]. These inconsistent trends indicate complex physics between the facility neutrals with thruster plasma or the Faraday probe that are not fully understood. Although the effect may be related to additional electron current fraction in the HET discharge, another possibility is related to probe sheath expansion when $R_{p} / \lambda_{D}$ is less than 50, as discussed in Sec. IV.B and in [59,60]. The ratio of $R_{p} / \lambda_{D}$ (where $R_{p}$ is the biased guard ring outer diameter) in Faraday probe measurements of the $200 \mathrm{~W}$ HET plume were estimated based on past studies [100]. The estimated ratio $R_{p} / \lambda_{D}$ varied from 21 at eight TDD to 15 at $20 \mathrm{TDD}$ at $3 \times 10^{-6}$ torr-Xe, and it increased to 64 at eight TDD and to 49 at 20 TDD at $3 \times 10^{-5}$ torr-Xe. Although this is still in the thin-sheath regime, it is possible that sheath expansion artificially increased Faraday probe measured ion current density at low pressure by several percent on the plume periphery; thus, the value of $\eta_{\text {Current }}$ extrapolated to zero pressure in Fig. 36a is overestimating the true current utilization as a thruster performance loss mechanism. It is noteworthy that the extrapolation of $\eta_{\text {Current }}$ to zero TDD in Fig. $36 \mathrm{~b}$ resulted in consistent
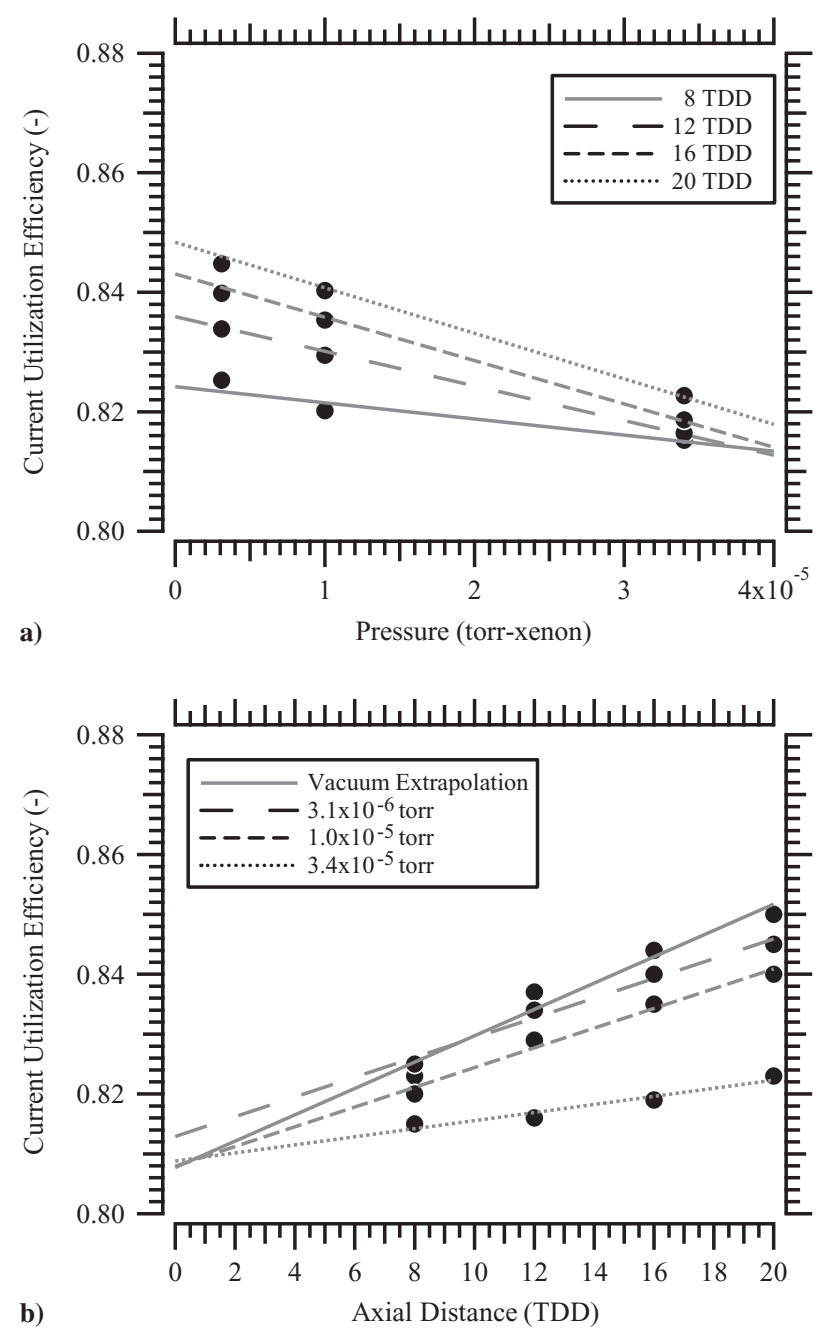

Fig. 36 Current utilization efficiency of a $200 \mathrm{~W}$ HET as a function of a) facility pressure and b) measurement distance. 
values for all background pressures, and it is further indication that the Faraday probe plume characterization with distance is necessary to determine global plume properties.

Based on the nature of facility effects on the plasma plume (i.e., ion scattering, ingestion) and Faraday probe (i.e., Bohm current, sheath expansion) measurements of far-field HET plume properties, the issues are expected to apply generally to the near-field and far-field of the HET and GIT plumes. Hence, the guidelines and recommendations in Secs. IV and $\underline{V}$ were aimed to characterize and correct for the overall facility pressure effects, including Faraday probe collection of Bohm current, ingested and ionized facility neutrals, ion scattering, and probe collector sheath expansion. If Faraday probe plume characterization with distance and facility pressure is a valid approach to correct and/or mitigate the overall facility effects, then a complete understanding of the individual processes may not be necessary. This result warrants further investigation, including different thruster technologies and thruster models, different facilities, and measurement region.

\section{Measurement Error and Uncertainty}

The total measurement error is the difference between a measured value and the true value, and it may be separated into a random error and a systematic error. Random error is stochastic or unpredictable in nature and may be associated with equipment sensitivity or signal noise; it may be reduced through repeated measurement, but not eliminated. The systematic error, or statistical bias, may be predictable and is typically constant or proportional to the true value. The systematic error may be known or unknown, and it may be associated with imperfect calibration, imperfect observation, or interference of the measurement process by the environment (i.e., facility effects). If the known systematic error is significant relative to the required measurement accuracy, a correction factor is appropriate to minimize the impact. Ideally, the systematic error is zero after correction factors are implemented; however, errors arising from imperfect corrections cannot be exactly quantified. Thus, there will be inherent errors in the correction factors, such as $\kappa_{G}, \kappa_{\mathrm{SEE}}, \kappa_{D}$, and $\kappa_{A}$. The error in these corrections contributes to overall measurement uncertainty. A detailed description of measurement error, uncertainty, and definition of terms and concepts may be found in $[101,102]$.

In Faraday probe measurements of ion current density in EP plumes, the random error is typically minimal compared to systematic error. The exception is on the plume periphery where the ion current density is orders of magnitude less than the central core and random error may be nonnegligible. The correction factors, test methodologies, and recommended analytical techniques in this guide have been established to allow characterizations and corrections that minimize systematic error and improve estimates of local charge flux and global EP plume properties in the space environment. A list of the primary systematic errors is provided in Table $\underline{2}$, along with the location of discussion. The magnitude of systematic measurement errors may vary for different experiments due to thruster technology,

\section{Table 2 Systematic errors in Faraday probe measurements}

\begin{tabular}{|c|c|}
\hline Parameter & Section \\
\hline Probe positioning and alignment & III.C \\
\hline Probe leakage current & $\overline{\text { III.D }}$ \\
\hline DAQ system and electrical configuration & III. $\overline{\mathrm{D}, \mathrm{III}} . \mathrm{E}$ \\
\hline Collector surface contamination & $\overline{\text { IIII.E }}$ \\
\hline Thermionic emission & $\overline{I V . B}$ \\
\hline Bias voltage and ion saturation & $\overline{\mathrm{IV} . \mathrm{B}}$ \\
\hline Sheath expansion & IV.B, V.E \\
\hline Bohm current collection & $\overline{\mathrm{IV} . \mathrm{B}}, \overline{\mathrm{V} . \mathrm{B}}$ \\
\hline Ion collection in collector-guard ring gap & V.A \\
\hline Collector secondary electron emission & $\overline{\mathrm{V} . \mathrm{A}}$ \\
\hline Hemispherical coordinate system & $\overline{\mathrm{V} . \mathrm{B}}$ \\
\hline CEX collisions with facility neutrals & $\overline{\mathrm{V} . \mathrm{B}}$ \\
\hline Facility neutral ingestion & $\overline{\mathrm{V} . \mathrm{B}}$ \\
\hline Divergence angle reference & V.B, $, \bar{V} . C, V . D$ \\
\hline Linear regression (to zero pressure, zero TDD) & $\mathrm{V} . \overline{\mathrm{B}}, \mathrm{E} . \overline{\mathrm{E}}$ \\
\hline Cathode plume & Appendix B \\
\hline
\end{tabular}

operating condition, experimental setup, and/or facility test conditions. In addition, there are limited flight comparisons with ground data and insufficient Faraday probe plume measurement in the space environment to rigorously determine the fidelity of linear regression to vacuum conditions or to zero TDD.

Based on this knowledge, experiments that follow the guidelines in Secs. III-V, as well as Appendix A, are expected to have less than approximately $\pm 5 \%$ uncertainty in far-field measurements and $\pm 10 \%$ uncertainty in near-field measurements. Local Langmuir probe measurements at the Faraday probe location enable direct calculation of the Bohm current and evaluation of the probe sheath, and they may be used to reduce measurement uncertainty. Comparing calculated global plume properties from Faraday probe measurements with other plasma diagnostics and evaluation with a thruster performance analysis may further increase confidence in results. For example, the GIT ion beam current measured by ion optics may be used to evaluate the Faraday probe integrated ion current density results. For a HET, the total ion beam current measured by a Faraday probe may be bounded by the thruster discharge current (maximum) and the lower limit based on evaluation of total thruster efficiency and utilization efficiencies [88]; this approach necessitates thrust measurements and other plasma diagnostics, such as electrostatic analyzers [1ㅡㄹ or ExB probes [1] .

\section{Probe Design Considerations}

\section{A. Design Guidelines}

Numerous investigations have evaluated Faraday probe designs and modifications aimed to form a uniform collector sheath and to prevent collection of low-energy ions generated through facility effects, such as CEX ions and ingested neutral particles [1,30,32]. These ion filtering mechanisms include collimators, electrically biased grids, or magnetic fields. Although these filtering approaches will successfully attenuate low-energy ions, they do not selectively isolate facility effects from the ionization of thruster and cathode neutrals downstream of the primary acceleration zone. For example, a collimated Faraday probe collects the low-energy thruster beam ion population, CEX ions, and ingested facility neutrals that are ionized and accelerated near the thruster exit. In contrast, the magnetically filtered Faraday probe or gridded Faraday probe eliminates all low-energy ion populations. Thus, the filtering mechanisms may not accurately determine the on-orbit plume characteristics. The recommended design is a nude Faraday probe with a guard ring, based on design simplicity and past systematic experiments that increase confidence in the ability to characterize systematic measurement errors.

A typical nude Faraday probe consists of a collector, guard ring, ceramic isolator, probe housing, and necessary fasteners and electrical wiring. The collector and guard ring are often made of metal with low sputter yield and low SEE, such as molybdenum, tungsten, or graphite $[49,78,79,104,105]$. Although materials spray coated with $2 \%$ thoriated tungsten have been used, it is not recommended due to a lower work function that leads to increased thermionic emission at a given temperature [65]. High-purity ( $\geq 99 \%)$ collector and guard ring materials should be used due to availability of literature on material properties. The collector and guard ring should be the same material to minimize differences associated with material properties, such as SEE and thermionic emission. The collector and/or guard ring electrical connections internal to the probe housing may be a suitable location to monitor a representative probe temperature for evaluation of effects associated with heating, such as thermionic emission.

The collector diameter is determined based on the expected range of ion current density, which may span microamperes per square centimeter in the far-field plume to milliamperes per square centimeter in the near-field plume of HETs and GITs; ion current density may exceed amperes per square centimeter in the far-field plume of pulsed electromagnetic thruster concepts, as discussed in Sec. VIII.C. Thus, the collector diameter should be scaled to achieve the necessary signal strength over the measured ion current density range in the near-field and far-field plumes for the experiment DAQ system capabilities, or voltage should be measured across a shunt resistor as described in Sec. III.D. In addition, the lowest achievable 
measurement resolution is set by the collector diameter $2 R_{C}$; thus, the collector diameter should be scaled to satisfy the experimental guidelines in Secs. IV.C and IV.D.

The gap width between the collector and guard ring may be less than 5 to $10 \lambda_{D}$ to create a flat, uniform sheath over the collector surface. Past experiments demonstrated that ions entering the gap between the nude Faraday probe collector and guard ring were collected by the walls, and they were a nonnegligible fraction of the measured collector current [77]. Although the effective ion collection area of nude Faraday probes is larger than cross-sectional geometric area of the collector, the area correction factor $\kappa_{A}$, as defined in Sec. V.B, has demonstrated consistent results for different nude probe configurations over a range of measurement distances and background pressures, where the isolator base of the gap between collector and guard ring was ceramic [18].

The ratio $R_{P} / \lambda_{D}$ is an important consideration in determining the range of plasma conditions where the thin-sheath assessment is valid. In a nude Faraday probe, the probe radius $R_{P}$ is equal to the guard ring outer diameter. The ratio $R_{P} / \lambda_{D}=50$ in Fig. 37 for $R_{P}$ from 0 to $100 \mathrm{~mm}$ over a wide range of plasma densities and electron temperatures is based on Eq. (2), showing the conditions where sheath expansion is negligible. Large $R_{P}$ enables Faraday probe measurements at larger $\lambda_{D}$ while satisfying $R_{P} / \lambda_{D}$ greater than 50, thereby enabling studies of lower plasma density at a given $T_{e}$. For example, $R_{P}$ of $40 \mathrm{~mm}$ enables Faraday probe measurements of $1 \mathrm{eV}$ plasma at density $10^{14} \mathrm{~m}^{-3}$, and $R_{P}$ of $100 \mathrm{~mm}$ is insufficient to measure a $1 \mathrm{eV}$ plasma at density of $10^{13} \mathrm{~m}^{-3}$.

There are two circumstances when a guard ring may not be viable. In regions of high plasma density, such as near-field measurements close to the plasma discharge, the gap between the collector and guard ring may be less than 5 to $10 \lambda_{D}$ and the gap design criteria is not satisfied. For instance, plasma density in the very near-field of HETs and GITs is $10^{17}$ to $10^{18} \mathrm{~m}^{-3}$, and the corresponding $\lambda_{D}$ is $17-50 \mu \mathrm{m}$ in a $5 \mathrm{eV}$ plasma based on Eq. (2). Thus, the gap between the collector and guard ring must be less than 0.1 to $0.5 \mathrm{~mm}$ to satisfy the 5 to $10 \lambda_{D}$ gap criteria. Under these conditions, there may be practical limits to the minimum gap width and significant challenges associated with probe fabrication, construction, assembly, alignment, and/or tolerance. A second situation where a guard ring may not be practical is one in which the physical probe diameter is large with respect to the region of interest, such as the near-field of a very small thruster, within or very near a HET discharge channel, or directly downstream of GIT grids before beamlet merging. Evaluation of gap widths larger than 5 to $10 \lambda_{D}$ was conducted in [77]. These experimental results indicated the 5 to $10 \lambda_{D}$ design criterion may be relaxed if the gap correction factor $\kappa_{G}$ was applied. It is recommended to evaluate these challenges and errors, as well as to compare to errors associated with collector sheath expansion if no guard ring is implemented. In the absence of plasma properties to estimate the range of $\lambda_{D}$, it is recommended to use a guard ring. Both approaches

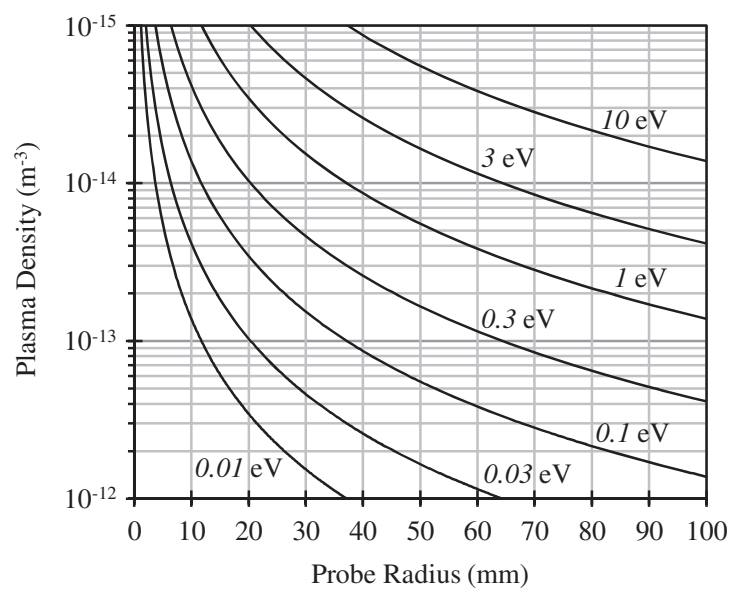

Fig. 37 Minimum plasma density measureable for a probe radius satisfying $R_{P} / \lambda_{D}$ greater than 50, with manifolds of fixed electron temperature. necessitate careful analysis and should be accounted for in the error analysis.

The isolator is intended to electrically isolate the collector and guard ring, and it is typically a dielectric, such as boron nitride (BN), Macor®, or aluminum oxide $\left(\mathrm{Al}_{2} \mathrm{O}_{3}\right)$. A comparison of dielectric materials used in probe construction for use in an EP plasma was provided elsewhere in the literature $[106,107]$. Boron nitride is available in multiple grades with high thermal conductivity, a temperature limit exceeding $3000 \mathrm{~K}$ (for XP grade BN), and excellent thermal shock resistance $[\underline{108,109}]$. Aluminum oxide, also known as alumina, has sufficient thermal conductivity and maximum temperature for most EP plasma plume environments, and it has a higher elastic modulus that is advantageous for applications with high mechanical and vibrational loads [110]. Macor is an easily machinable glass ceramic with the low thermal conductivity, no porosity, and no outgassing [111]. In general, the ceramic isolator SEE and sputter yields should be minimized; these properties may be found in the literature $[48,50,107,112]$. However, other test considerations may drive material selection. For example, BN may have advantages associated with thermal properties and alumina may have beneficial mechanical properties for rapid probe positioning.

The probe housing should be capable of withstanding the plasma environment, and thus it is made from a material with a low sputter yield, such as graphite [55,105]. Alternative materials such as aluminum may have advantages for probe construction; however, the aluminum sputter yield due to xenon bombardment is approximately five times higher than graphite [105]. Kapton [48] may be used to cover the housing material, but it will undergo degradation in the plasma environment. As stated in Sec. III.E, Faraday probe electrical wiring and fasteners should not be exposed to the plasma in order to minimize sputtering, deposition, and degradation. A shield may be implemented to limit direct ion beam impingement.

\section{B. Recommended Probe Design}

Based on the design guidelines in Sec. VII.A, a recommended nude Faraday probe design is shown in Fig. 38. The diagram shows major components of the probe design and includes key features based on past test campaigns. It should be noted that this design is notional, and it has not been fabricated or evaluated with thruster plasma in a ground test environment. The collector, guard ring, isolator, and probe housing are shown in Fig. 38a. Probe materials discussed in Sec. VII.A would suffice for the design. However, for consistency in results associated with material properties (i.e., SEE, sputter yield, work function), it is advised to use tungsten due to an extensive knowledge of material properties and plasma interactions. The recommended isolator material in Fig. 38 is BN due to thermal characteristics and knowledge of material properties based on past studies $[48, \underline{50}, 108,109]$. Alternative isolator ceramics may be used for specific advantages, such as mechanical properties.

Probe manufacturability has been considered, including separating the isolator into two components for alignment and the notched isolator base as shown in Fig. 38b. The notched isolator base is to reduce the buildup of deposited material, and thereby reduce the possibility of electrical leakage current caused by a conductive path between the collector and guard ring. In addition, there are through holes in the isolator and tapped holes in the collector and guard ring, which enable options for probe assembly and electrical connections at fasteners internal to the probe housing. This configuration is also expected to allow adjustments in component alignment, including concentricity between the collector and guard ring. The diagram does not specify an attachment between the isolator and probe housing. Vent holes are included at the base of the ceramic isolator in Fig. 38a to reduce the buildup of neutral particles in the gap; however, this may not be necessary for low particle flux in the far-field plume.

Critical design dimensions for the probe in Fig. 38 are shown in Fig. 39a with respect to recommendations for near-field and far-field measurements in Sec. IV, including the collector diameter, the gap width between the collector and guard ring, and the guard ring outer diameter. These critical dimensions should be evaluated and satisfied throughout the plume measurement range. For a given investigation, 


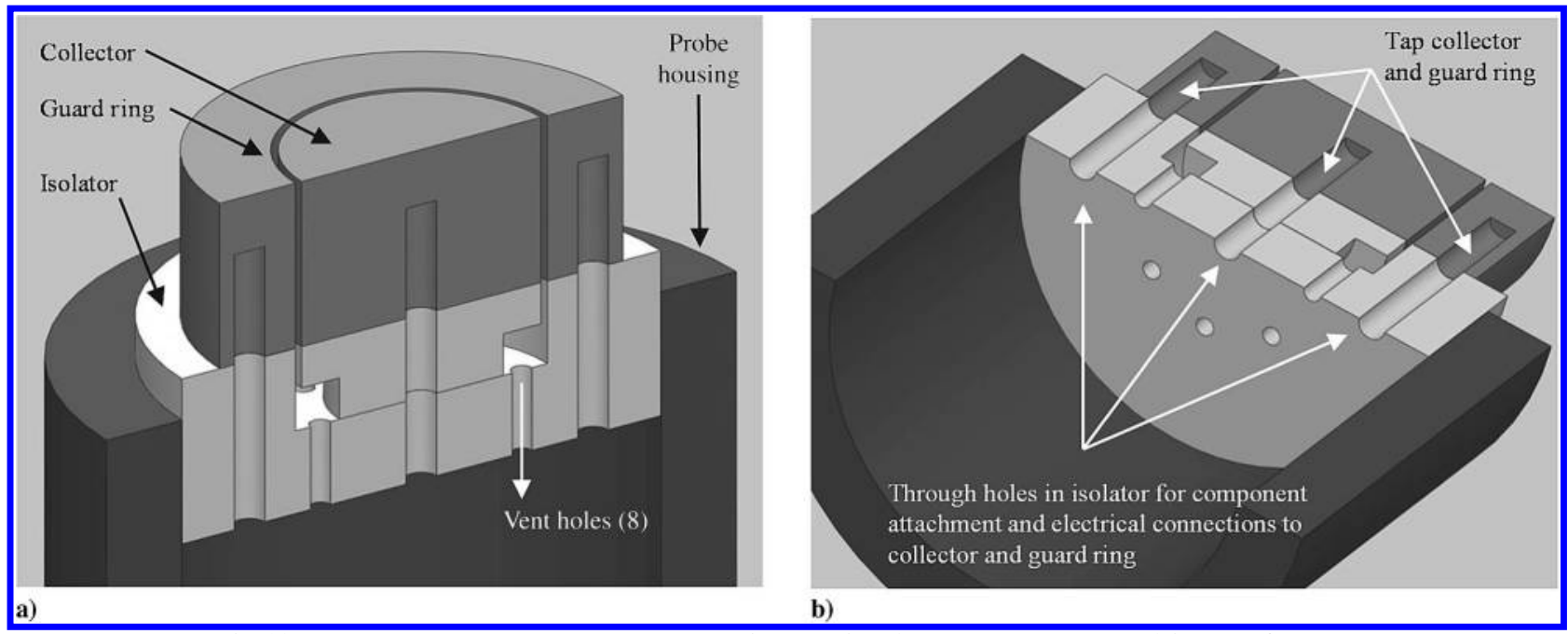

Fig. 38 Recommended nude Faraday probe design showing a) key components and b) internal features.

$\lambda_{D}$ is typically lowest at the highest thruster discharge current condition and at the nearest downstream measurement location; this value should be used for scaling the gap width. The ratio $R_{P} / \lambda_{D}$ is typically smallest at the lowest thruster discharge current condition and lowest pressure on the plume periphery; this value should be used for scaling the guard ring outer diameter.

Dimensions for two recommended probe designs are provided in Fig. 39b. For $2 R_{C}$ of $17 \mathrm{~mm}$, the far-field measurement resolution is $1 \mathrm{deg}$ at $1 \mathrm{~m}$ and $2 \mathrm{deg}$ at $0.5 \mathrm{~m}$. The gap width is set to $0.5 \mathrm{~mm}$, which is suitable for $\lambda_{D}$ greater than $0.05 \mathrm{~mm}$. Two outer diameter guard rings are shown in Fig. $39 \mathrm{~b}$ for different values of $R_{P}$. A guard ring outer diameter of $106 \mathrm{~mm}\left(\overline{R_{P}}=53 \mathrm{~mm}\right)$ corresponds to $R_{P} / \lambda_{D}$ greater than 50 at greater than $5 \times 10^{13} \mathrm{~m}^{-3}$ in a $1 \mathrm{eV}$ plasma. The guard ring outer diameter of $34 \mathrm{~mm}\left(R_{P}=17 \mathrm{~mm}\right)$ corresponds to $R_{P} / \lambda_{D}$ greater than 50 at greater than $5 \times 10^{14} \mathrm{~m}^{-3}$ in a $1 \mathrm{eV}$ plasma, where the smaller probe cross section may reduce probe heating and thruster perturbations.

The Faraday probe is connected with fasteners that attach the collector and guard ring to the isolator assembly. In addition, the fasteners may be used as attachment points for electrical connections and thermocouples within the probe housing. A thermocouple is recommended at the guard ring electrical connection to monitor probe temperature. The probe housing may extend greater than $50 \mathrm{~mm}$ from a mounting post or diagnostic array. The isolator extends upstream of the probe housing in order to reduce deposition and the possibility of a current path forming across the isolator surface between the guard ring and conductive probe housing. In general, these probe dimensions may be modified to improve manufacturability, or as needed, for experimental requirements.

In situations where the probe diameter is minimized or a guard ring is not used, several design options have been implemented $[7,21,44,54,113]$. For simplicity, the planar Faraday probe design used in [113] is the recommended approach, where the collector is flush mounted with an isolator jacket. This is due to a known exposed collection area with no possibility of ion collection on the sidewalls, as occurs in the probe configuration in Figs. 38 and 39. The sheath effects should be evaluated as stated in Sec. VII.A with local Langmuir probe measurements, and probe heating may be monitored with a thermocouple. Techniques for construction and assembly of small plasma probes were found elsewhere [45,106,114].

Evaluations of the plume periphery for high-fidelity predictions of ion flux in the space environment may necessitate a larger collector to improve the signal-to-noise ratio and a larger guard ring to satisfy $R_{P} / \lambda_{D}$ greater than 50. The scaling shown in Fig. 39a should be used to scale this probe design. In addition, local Faraday probe measurements are recommended to evaluate sheath expansion and Bohm current, as discussed in Sec. IV.B.

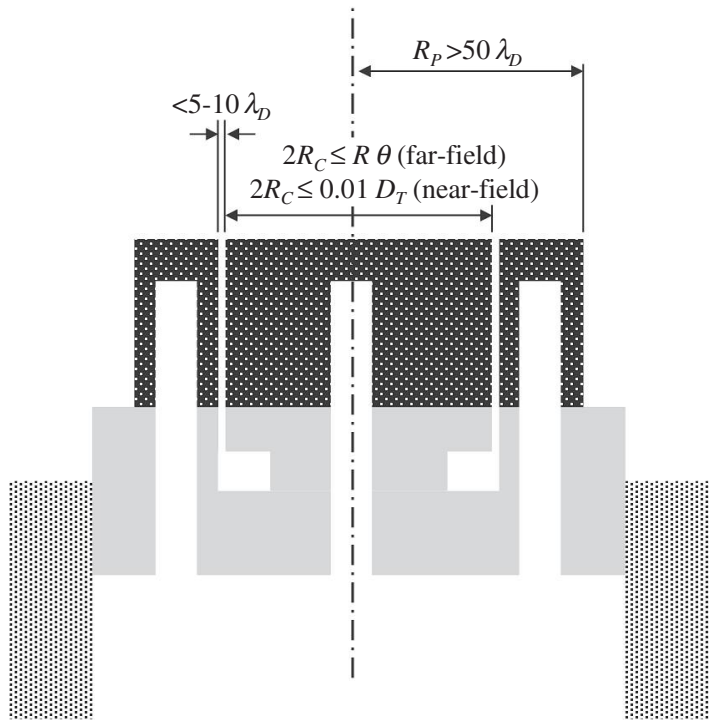

a)

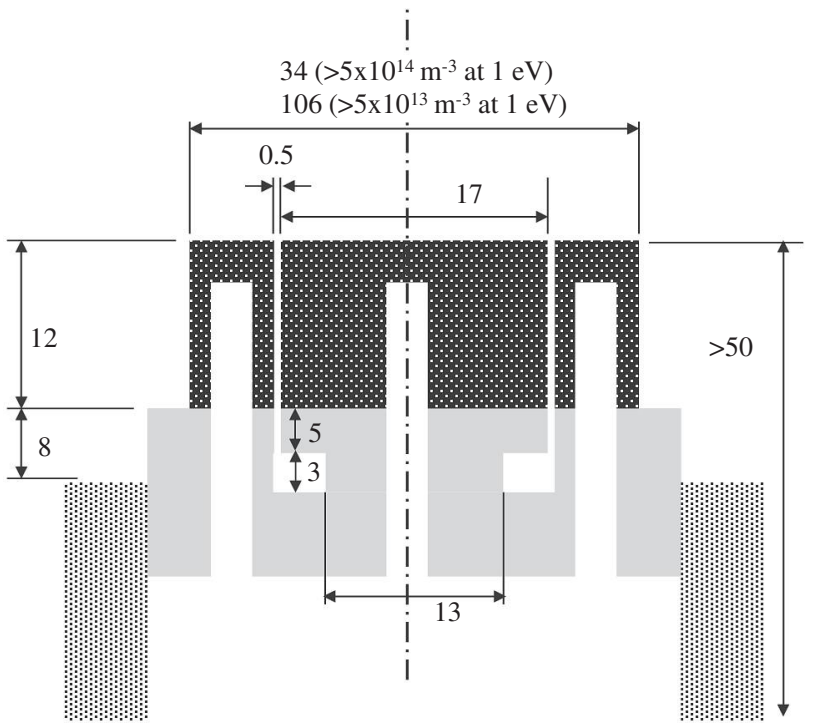

b)

Fig. 39 Recommended nude Faraday probe design showing a) key design parameters and b) a design dimensions in mm. 


\section{Considerations for Other EP Technologies}

The Faraday probe experimental apparatus, test methodologies, and analysis techniques presented in Secs. III, IV, and V, respectively, are applicable to many EP technologies. Multiple criteria in Table A1 of Appendix A are common to all EP devices. These are primarily related to probe considerations and experimental apparatus. However, there is limited information in the literature on Faraday probe measurements for the EP devices in this section, and it is undetermined if additional measurement considerations or analysis techniques are warranted. This is especially true for issues related to thruster interactions and plume characterizations with distance and pressure. Limitations of the guidelines in Table A1 and modifications for select EP technologies are described in the following.

\section{A. Electrospray Thrusters}

Electrospray propulsion technologies, which includes field emission electric propulsion and colloid thrusters, typically use a small number (less than 10) of emission sites and small emission area for low-power sub-millinewton thrust applications $[115,116]$. There is limited information in the literature of Faraday probe measurements for single emitter experiments or electrospray arrays with a small number of emitters; thus, it is unclear if additional Faraday probe considerations will arise in measurements of large electrospray arrays for greater than microampere-level devices [117].

Plume measurements of the colloid micronewton thruster (CMNT) system, a nine-emitter array, were conducted for the NASA Space Technology 7-Disturbance Reduction System (ST7-DRS) flown on the Laser Interferometer Space Antenna Pathfinder mission. The CMNT beam was not evaluated with a traditional Faraday probe; however, a segmented plume target in the far-field plume provided details of the ion current density profiles approximately $34 \mathrm{~cm}$ downstream of the thruster $[115,117]$. The plume target consisted of 64 electrometers in a cross configuration along the $x$ axis and $y$ axis, as shown in Fig. 8 for the cylindrical coordinate system. There were minimal details on the experimental apparatus or facility configuration, and the plume measurements were not consistent with the test methods in this paper. However, the reported ion flux and divergence half-angles in Fig. 40 indicate the electrospray ion beam is similar to GIT and HET plumes. Figure $40 \mathrm{~b}$ demonstrate the $99.9 \%$ divergence half-angles were within $50 \mathrm{deg}$ from the thruster centerline, and the $95.5 \%$ half-angle was no greater than $24 \mathrm{deg}$ in any operational condition [115]. The integrated ion beam current was not compared to electrospray emitted current.

Based on the ST7-DRM dataset, Faraday probe guidelines in this paper should be used as a starting point for measurements of electrospray plumes. The influence of facility background pressure or facility electrical environment has not been established for electrospray thruster technologies. However, the plume characterization with multiple background pressures and measurement distances are advised, as listed in Table A1, to isolate facility effects associated with CEX collisions, Bohm current collection, and probe sheath expansion. Electrosprays may be operated in several configurations to achieve beam neutralization, including with a neutralizer cathode, with both positive and negative emitters, or operated in a bipolar fashion with alternating positive and negative particles. The latter two approaches may require additional considerations that are beyond the scope of this paper. In addition, Faraday probe design should be evaluated for measurements of the electrospray plasma with charged droplets, including guidelines for the guard ring and gap spacing. As stated in Sec. II.A, the effect of propellant on the Faraday probe surfaces should be considered if an ionic liquid or condensable propellant is used.

\section{B. Arcjet Thrusters}

Electrothermal thrusters, such as arcjets and resistojets, use electric heating to impart energy to the propellant and accelerate through a nozzle to produce thrust $[118,119]$. In the arcjet, an arc originating from the cathode attaches to the anode nozzle to heat the propellant, which can then be accelerated through the nozzle to produce thrust. The arcjet flow conditions evolve from nearly fully ionized plasma near the cathode to a relatively cold plasma near the anode surface, and a weakly ionized plasma in the exhaust plume. Arc current density near the arcjet cathode
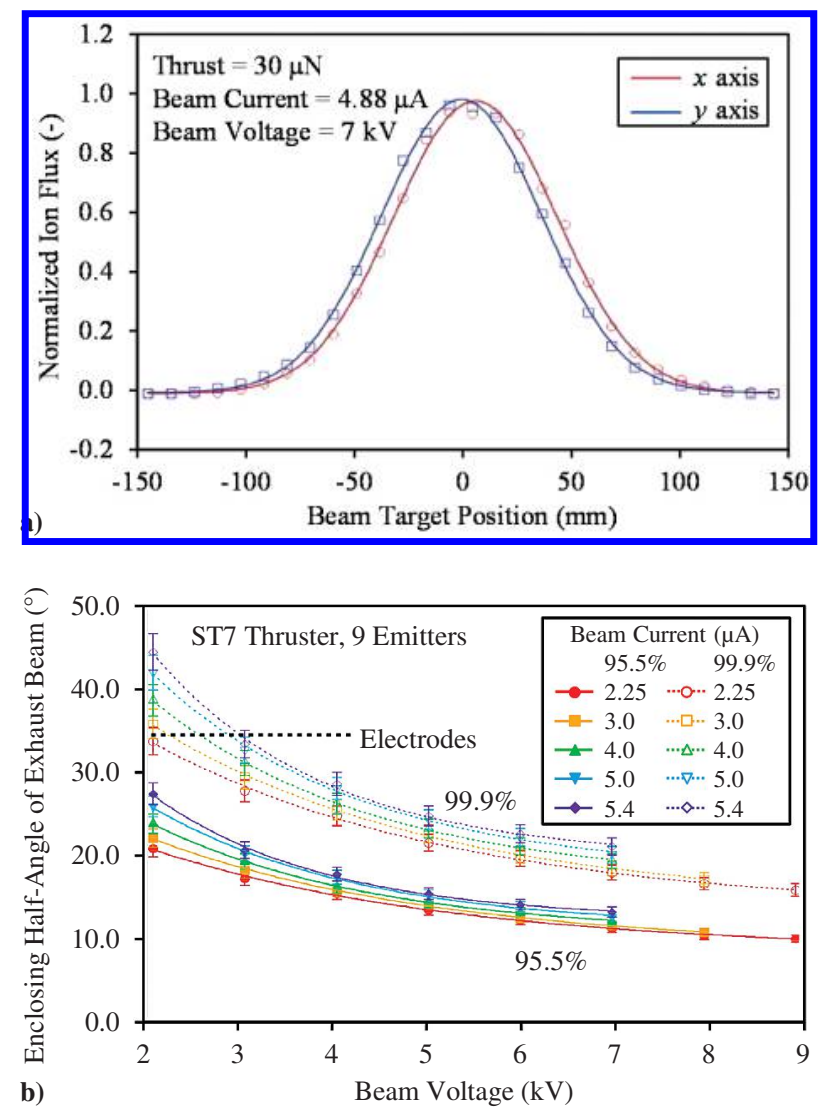

Fig. 40 Far-field plume measurements of the ST7-DRS electrospray thruster a) normalized ion flux recreated from [117] and b) divergence half-angle recreated from [115].

has been estimated at $10^{4} \mathrm{~A} / \mathrm{cm}^{2}$, and peak current density along the anode surface was measured to be greater than $10 \mathrm{~A} / \mathrm{cm}^{2}[118,120]$. There have been multiple studies of the arcjet exit plane, near-field plasma, and far-field plasma [121-124]; however, there is a dearth of information on ion current density in the arcjet plume due to the very low ionization fraction of less than $10^{-3}$. Plasma properties in the arcjet plume are an electron temperature less than $1 \mathrm{eV}$ and electron density ranging from less than $10^{13} \mathrm{~cm}^{-3}$ in the near-field plume to less than $10^{9} \mathrm{~cm}^{-3}$ in the far-field plume $[118,121]$. Thus, the signal-to-noise ratio may be a challenge and the orbital-motion-limited sheath assessment may be necessary, as opposed to the thin-sheath assessment, due to the large $\lambda_{D}$ at low plasma density. Neutral propellant temperatures may approach 3000 to $5000 \mathrm{~K}$ near the arcjet exit plane, and the exhaust velocity ranges from hundreds of kilometers per second up to approximately $1000 \mathrm{~km} / \mathrm{s}$ on the thruster centerline, depending on propellant, where the ratio of ion velocity to neutral exhaust velocity is $\sim 1.5[122,125]$.

The Faraday probe guidelines and recommendations in this paper may be used as a starting point; however, an examination of the arcjet near-field and far-field plumes is necessary to determine whether the guidelines and recommendations are suitable. It is not known to what extent facility effects influence the arcjet plume; they may be minimal because most of the acceleration is within the nozzle area and the ionization fraction is very low in the plume. To establish guidelines for Faraday probe measurements of arcjet thrusters, examination of ion current density with characterization of background pressure and downstream distance is advised. These data should be correlated to measurements of plasma properties near the exit plane and far-field plume, and they should be considered with respect to Faraday probe design requirements and design applicability throughout the plume. For example, the Debye length may range from $3 \times 10^{4} \mathrm{~cm}[\underline{122}]$ near the arcjet exit plane to the order of $10^{-2} \mathrm{~cm}$ in the far-field plume [126]. To accommodate these conditions, the gap width of a nude Faraday probe collector must be less than $0.03 \mathrm{~mm}$ and is not practical. Based on past plasma measurements of the arcjet plume, probe heating must 
also be considered and recommendations for probe operating characteristics in Sec. IV.B should be implemented.

\section{Electromagnetic Thrusters}

Faraday probe measurements in the near-field and far-field plumes of electromagnetic propulsion are challenging due to the severe plasma plume environment, the wide range of plasma properties, and the complex transient nature of electromagnetic thrusters. Common electromagnetic thruster devices include magnetoplasmadynamic thrusters (MPDTs) and pulsed plasma thrusters (PPTs). Electromagnetic thruster concepts can range in average power from less than $1 \mathrm{~W}$ up to megawatt levels, and total enthalpies in the plume can reach $5 \times 10^{8} \mathrm{~J} / \mathrm{kg}$. Peak pulsed ion current density in these devices may exceed $10^{6} \mathrm{~mA} / \mathrm{cm}^{2}$ near the plasma discharge and be greater than $10^{3} \mathrm{~mA} / \mathrm{cm}^{2}$ in the far-field plume [127-130]. At these extreme conditions, the probe is likely to experience elevated temperatures and the recommendations for probe operating characteristics in Sec. IV.B should be implemented. There have been few comprehensive studies of ion current density in the plume of MPDTs or PPTs. In cases where ion current density is reported, it is typically measured with Langmuir probes or Hall probes [127-129].

Ion current density measurements using a planar Langmuir probe are shown in Fig. 41 from investigations of the Lincoln Experimental Satellite (LES) $\overline{8} / 9$ PPT plume [128]. These ion current density measurements were conducted in a hemispherical coordinate system at a radius of $24 \mathrm{~cm}$ with a planar probe rake, which corresponds to $R=9.4$ TDD for the $2.54 \times 2.54 \mathrm{~cm}^{2}$ exit cross section. The planar probes had copper collectors and a guard ring, both biased to $-40 \mathrm{~V}$ with respect to facility ground. In Fig. 41, the relatively flat ion beam profile on the thruster centerline shows a peak ion current density of approximately $2000 \mathrm{~mA} / \mathrm{cm}^{2}$, which is two to three orders of magnitude larger than HETs or GITs at a similar location in the far-field plume ( 8-10 TDD). Based on the results with a planar Langmuir probe, a Faraday probe designed for the high-energy flux in Fig. 41 may be viable for examinations of the PPT plume. However, the proper test approach, data analysis, and characterization of facility effects on Faraday probe measurements in the PPT plume has not been established. For example, several PPT studies with Langmuir probes have confirmed asymmetry in plasma plume properties downstream of the exit plane, which were attributed to the plane parallel or perpendicular to the rectangular PPT discharge cross section [131]. Thus, the Faraday probe guidelines and recommendations in this paper should be evaluated before adoption and/or modification for the PPT plume.

A representative measurement of ion beam current in the very nearfield of an MPDT operating at $60 \mathrm{~kW}$ and $1500 \mathrm{~A}$ is shown in Fig. 42 [129]. This measurement was conducted with a Hall probe, where the self-induced magnetic field distribution is related to the enclosed current. The Hall probe was swept through the plume within $\sim 50 \mathrm{~ms}$ at fixed $2.7 \mathrm{~cm}$ downstream of the exit plane, where the anode outer radius was $\sim 2.54 \mathrm{~cm}$, as shown in the diagram within Fig. 42 . In this configuration, the cathode was recessed within the discharge region and

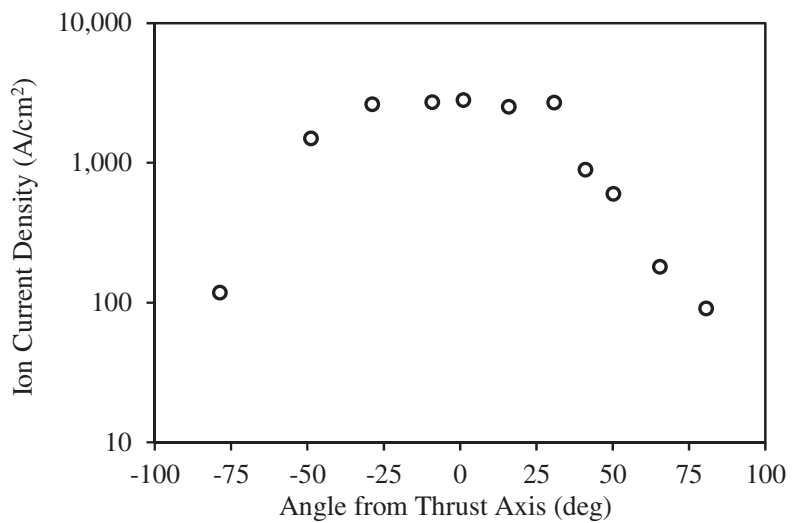

Fig. 41 Far-field ion current density measurements of the LES 8/9 PPT with a rake of planar Langmuir probes, recreated from [128].

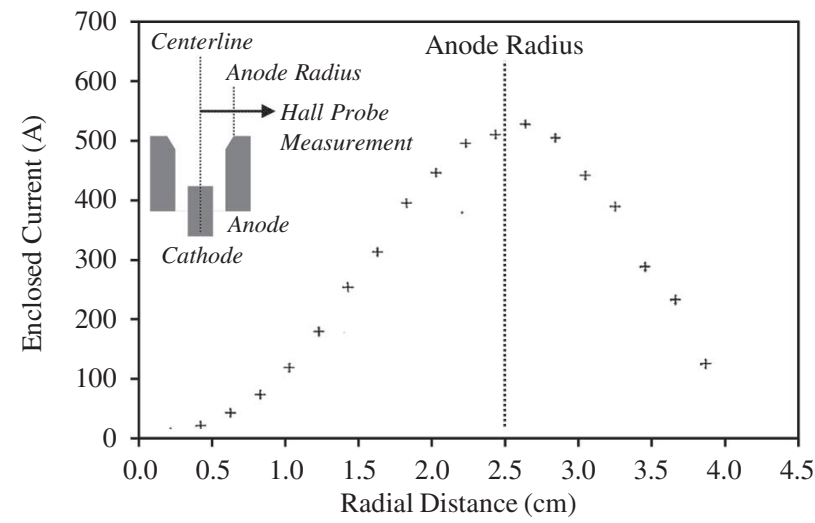

Fig. 42 Enclosed current distribution of the MPDT plume at $2.7 \mathrm{~cm}$ downstream of the exit, recreated from [129].

oriented along the thruster centerline $(r=0 \mathrm{~cm})$. The peak current within the Hall probe loop was near the anode radius, indicating the current was attached to the anode face. Multiple studies have reported that the applied-field MPDT was highly susceptible to facility effects, such as propellant ingestion and ion scattering in the plume, since the acceleration region can extend far downstream of the thruster exit [132-134]. Although facility effects have been evaluated experimentally and computationally, there is not a standard approach to mitigate the facility effects on MPDT performance or plume evaluations, and such an analysis is beyond the scope of this paper. A systematic and comprehensive evaluation of the MPDT near-field and far-field plumes is necessary to determine whether the guidelines and recommendations in this paper are suitable for the MPDT plume. This will necessitate Faraday probe measurements of ion current density, characterization of background pressure and downstream distance, and plume examination of plasma properties throughout the plume (i.e., electron density, electron temperature, ion species) to inform Faraday probe design requirements and design applicability throughout the plume.

\section{Conclusions}

This paper described guidelines and recommended practices for the use of Faraday probes to measure ion flux in the near-field and far-field plumes of EP devices: specifically, HETs and GITs. These measurements were used to quantify thruster performance loss mechanisms and global beam properties, as well as to evaluate plasma plume interactions with the host spacecraft. No viable approach has been demonstrated to quantitatively predict the influence of facility pressure effects throughout the EP plume a priori. Rigorous Faraday probe studies of far-field Hall thruster plumes have advanced understanding of the facility effects on thruster plasma and probe ion collection behavior, and they have led to test methods, analysis techniques, and correction factors to reduce systematic measurement errors. A measurement uncertainty less than approximately $\pm 5 \%$ in far-field measurements and $\pm 10 \%$ in near-field measurements was possible, and it may be improved with local Langmuir probe measurements or evaluation with thruster telemetry and efficiency analysis. Further investigation is warranted to better understand facility effects on the near-field plume region and data analysis approaches to improve plume predictions of the space environment. The state of knowledge of Faraday probe measurements for other EP concepts is limited, including electrosprays, arcjets, and electromagnetic thrusters. Thus, the guidelines and recommendations described in this paper should serve as a starting point.

The establishment of recommended test practices for Faraday probe measurements will enable quantitative evaluation of EP plume properties and enhance the quality of comparisons between different EP devices and facilities. This will broaden the acceptance of Faraday probe results and improve measurement fidelity for on-orbit predictions and model validation. Standardization and improved 
predictive capabilities have broad application to laboratory researchers, satellite designers, and operators, and they ultimately advance the utilization of EP for flight.

\section{Appendix A: Summary of Recommended Practices}

The numerous guidelines and recommendations for Faraday probe test practices are summarized in Table A1 for HETs and GITs, including the relevant section where additional information is provided.

\section{Appendix B: Method of Cathode Plume Removal}

In the case of a centrally mounted cathode, the recommended approach is to remove the contributions of the cathode plume from the Faraday probe results. One approach is to evaluate the cathode plume as an expanding jet into vacuum that does not interact with itself or the thruster ion beam. The approach will be demonstrated based on analysis of the NASA-300M at $500 \mathrm{~V}, 20 \mathrm{~kW}$ in Figs. B1-B3 [45]. The first step is to identify a region near the cathode where the measured ion current is dominated by the cathode plume. In past studies, the cathode plume region was defined as $Z$ less than 0.2 TDD in the radial vicinity

Table A1 Summary of Faraday probe guidelines and recommendations for HETs and GITs

\begin{tabular}{l}
\hline Experimental parameter/section \\
Coordinate geometry/III.A, III.B
\end{tabular} Background pressure characterization/
IV.E

Measurement distance/IV.C, IV.D

Spatial characterization/IV.E Measurement span/IV.C, IV.D

Measurement resolution/IV.C, IV.D

Probe materials/VII.A

Collector bias voltage/IV.B

Probe electrical resistance/III.D, III.E

Diagnostic alignment/III.E

Bohm current/IV.B

Thermionic emission current/IV.B

Probe checkout/III.E, IV.B

Probe cabling/III.E

Thruster operation/IV.A

Probe design dimensions/ㅍI

Correction for collector SEE/V.A

Corrections for hemispherical coordinate system/V.B

Correction for probe collection area/V.A
Guideline

1) Channel or grid outer diameter $D_{T}$ should be used for HETs and GITs, respectively. ${ }^{a}$

2) External cathode should be located at $\Phi=90 \mathrm{deg}$ (or $270 \mathrm{deg}$ ) for measurement in $\Phi=0 \mathrm{deg}, 180 \mathrm{deg}$ plane.

3) Hemispherical coordinate system should be used in far-field.

4) Cylindrical coordinate system should be used in near-field.

1) Measurements should be conducted at a minimum four background pressures.

2) Pressure characterization should include plume measurements at the lowest achievable facility pressure during thruster operation.

3) Pressure should follow recommendations in [57].

1) Far-field measurements should be greater than four TDD.

2) Near-field measurements should be less than four TDD.

3) Near-field HET analysis should be upstream of the transitional region and may be greater than 0.2 TDD.

Measurements should be conducted at a minimum four distances at all background pressures.

1) Far-field measurements at fixed $R$ should $\operatorname{span} \theta=0$ to $90 \mathrm{deg}$, for fixed $\Phi$ and at $\Phi+180 \mathrm{deg}$ for the opposite side of the plume (e.g., $\theta=0$ to $90 \mathrm{deg}$ at $\Phi=0$ and $180 \mathrm{deg}$ ). ${ }^{\mathrm{d}}$

2) Near-field measurements at fixed $Z$ should extend to the location where measured ion current density is less than $0.2 \%$ of the maximum value along the radial profile, for fixed $\Phi$ and at $\Phi+180 \mathrm{deg}$ for the opposite side of the plume (e.g., $\Phi=0$ and $180 \mathrm{deg}$ ).

1) Far-field angular resolution should be $\left(2 R_{C} / R\right) \leq d \theta \leq 2$ deg.

2) Near-field radial resolution should be $2 R_{C} \leq d r \leq 0.01 D_{T}$ or $\leq 1 \mathrm{~mm}$, whichever is greater.

1) Probe collector, guard ring, and side surfaces should be $\geq 99 \%$ purity molybdenum, graphite, or tungsten.

2) Isolator materials may be boron nitride, aluminum oxide, or Macor. ${ }^{f}$

1) Probe collector and guard ring should be biased voltage relative to ground to achieve ion saturation throughout the measurement region.

2) Bias voltage to achieve ion saturation should be characterized at multiple locations in the plume to span the maximum and minimum $n$ and $T_{e}$ (e.g., minimum $R$ at $\theta=0 \mathrm{deg}$ and the maximum $R$ at $\theta=90 \mathrm{deg}$ in the far-field).

3) Collector and guard ring bias voltage should be equal.

1) Collector resistance to ground and $R_{\mathrm{C}-\mathrm{GR}}$ should be measured before testing, and they should exceed $100 \mathrm{M} \Omega$

2) Collector shunt resistor may range from 10 to $1000 \Omega$.

1) Probe alignment should be conducted before pumping the facility to vacuum conditions

2) Collector face should be oriented parallel to the thruster exit plane within $1 \mathrm{deg}$, when positioned on thruster centerline.

3) Probe position accuracy should be within $1 \mathrm{~mm}$ or within $0.5 \% R$ (or $Z$ ) at the maximum measurement distance (e.g.,

$5 \mathrm{~mm}$ at $R=1 \mathrm{~m}$ ), whichever is greater.

Bohm current density to the collector should be less than $1 \%$ of the measured ion current density.

Collector thermionic emission current should be less than $1 \%$ of the measured ion current density.

1) No obstructions should be in the line of sight of the Faraday probe to any point of the thruster plasma discharge.

2) All probe mounting structures and cables near the probe should be downstream of the probe collection surface.

3) Probe mounting structure that experiences direct beam ion impingement should be shielded with low-sputter materials, such as Kapton or graphite.

4) May conduct visual inspection and electrical verification of the probe at the beginning and end of a test campaign, or if there is a change in measurement repeatability over time.

1) Probe cabling should be coaxial cable or twisted shielded pair, with no electrical leads or connections exposed to plasma.

2) Cable shielding should be grounded to the facility walls in ground testing.

1) Thruster discharge should reach operational steady state before conducting measurements (user defined).

2) Thruster telemetry should be monitored for perturbation during measurements.

1) Collector to guard ring gap should be less than $5 \lambda_{D}$ to $10 \lambda_{D}$.

2) Ratio $R_{P} / \lambda_{D}$ should be greater than 50 .

3) $2 R_{C}$ should be less than or equal to $R \theta$ in the far-field.

4) $2 R_{C}$ should be less than or equal to $0.01 D_{T}$ in the near-field.

Measurements should be corrected for collector SEE with $\kappa_{\mathrm{SEE}}$.

Far-field measurements in a hemispherical coordinate system should be corrected with $\kappa_{A}$ and $\kappa_{D}$.

Probe ion collection area should be corrected for ions entering gap between the collector and guard ring with $\kappa_{G}$.

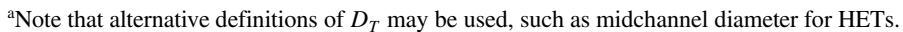

${ }^{\mathrm{b}}$ Note that pressure recommendations are the same for all working gases. For increased accuracy, recommend lower background pressure and additional pressure characterizations. Qualitative assessment of plume profile may be conducted at a single background pressure.

'Note that, for increased accuracy, recommend additional distances and additional background pressures. Qualitative assessment of plume may be conducted at a single distance.

${ }^{\mathrm{d}}$ Note that, for increased accuracy, recommend $\pm 100 \mathrm{deg}$ for far-field measurements.

eNote that, for increased accuracy, recommend $\leq 1$ deg resolution for far-field measurements.

${ }^{\mathrm{f}}$ Note that recommended probe design lists specific materials for consistency in Sec. VII.B. Alternative materials may be selected for specific applications. 

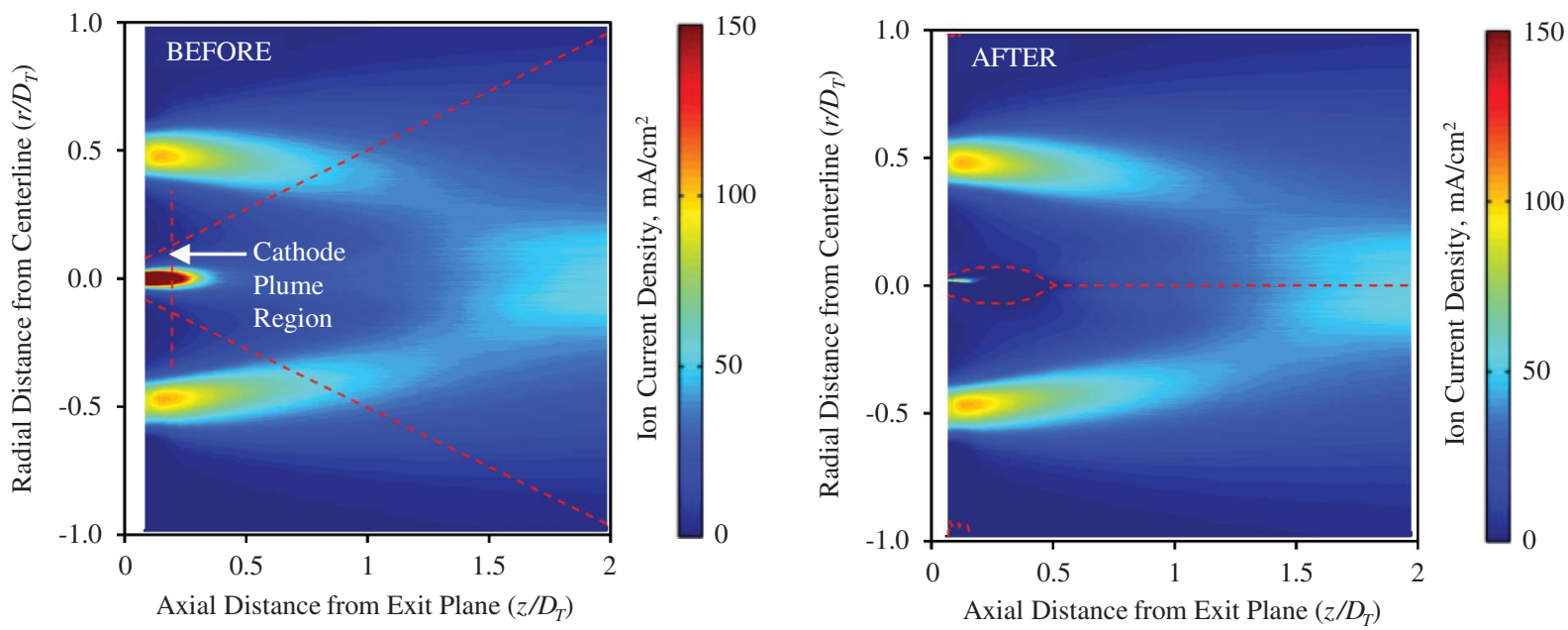

a)

b)

Fig. B1 Ion current density from a) plume measurements and b) with cathode plume removal, recreated from [45].

of the cathode plume $[45,54]$, which is within approximately $0.1 D_{T}$ in Fig. B1a, although the range can be modified. The dashed lines in Fig. $\overline{\mathrm{B} 1 \mathrm{a}}$ are overlaid to show the approximate boundaries of the cathode plume region in the analysis of [45].

Analyses of the NASA-457Mv2 in Fig. 26a and NASA-300M in Fig. B1a demonstrated that an exponential decay function of the form in Eq. (B1) yielded an excellent fit to the radial cathode plume profile at fixed $[\underline{45}]$ :

$$
f(r)=C_{1} e^{-|r| / C_{2}}
$$

The cathode plume expands as it travels downstream, where the parameters $C_{1}$ and $C_{2}$ are constant for a given radial plume profile at a fixed $Z$ but may vary with axial location in the cathode plume region. The recommended approach is to fit Eq. (B1) at multiple axial positions within the measured cathode plume region. Past studies used a method of least squares to determine $C_{1}$ and $C_{2}$ at each axial measurement location, indicated by markers in Figs. B2a and B2b. Based on the values of $C_{1}$ and $C_{2}$ for the exponential decay function in Eq. (B1), the radial cathode current density profiles may be integrated to calculate the total cathode plume current at each downstream distance, as indicated by markers in Fig. B2c. This calculation allows determination of a mean plume current in the expanding cathode jet, which is assumed constant with downstream distance in the cathode plume region. The mean cathode plume current is approximately $0.684 \mathrm{~A}$, as shown in Fig. B2c. To determine the cathode plume ion current density throughout the Faraday probe measurement region, it is necessary to develop an expression for parameters $C_{1}$ and $C_{2}$ as a function of axial location in the cathode plume. To this end, a second-order polynomial is used to evaluate variation of $C_{2}$ as a function of $Z$ in Fig. B2b. Using this second-order polynomial expression for $C_{2}$ and the mean cathode plume current in Fig. B2c, an expression for $C_{1}$ can be determined as a function of $Z$ with $\overline{\mathrm{Eq}}$. (B1), shown as a dashed line in Fig. B2a. The calculated values of $\overline{C_{1}}$ for an expanding cathode jet (dashed line) in Fig. B2a are consistent with the values determined with least-squares analyses (markers), thereby indicating the approach is suitable to evaluate the cathode plume ion current density throughout the measurement domain.

The cathode plume profile developed using Eq. (B1) and Fig. B2 may then be subtracted from the Faraday probe ion current density measurements. Based on the total cathode plume current in Fig. B2c, the cathode plume contributed $\sim 0.68$ A current to the integrated thruster ion beam current, which was $\sim 2 \%$ of the thruster discharge current. However, removal of the cathode plume has a larger impact on evaluation of beam divergence, including the iterative pathfinding method described in Sec. V.C. This is due to the elevated current density outside of the channel, which would alter values of $r_{0}$ and $z_{0}$ in the free-expanding jet in Fig. 27.
The cathode plume model is shown for the NASA-300M HET at various axial positions in Fig. B3, where solid black lines indicate the raw trace, and dashed red lines indicate the curve-fit results [45]. The flooded ion current density plots after cathode plume removal are shown in Fig. B1b, where dashed lines indicate the limits of integration. Spurious spikes may be present in the postsubtraction data due to minor
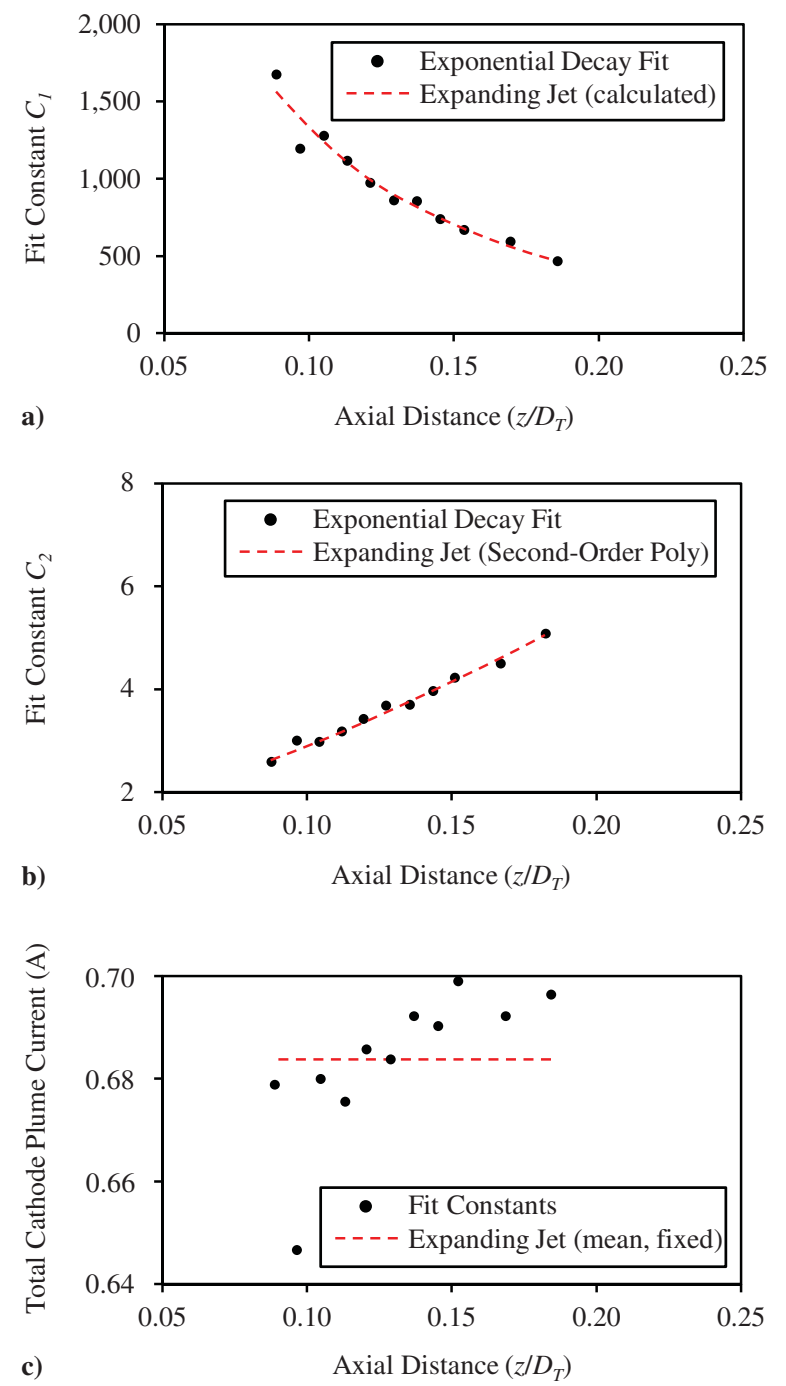

Fig. B2 Curve-fit parameters a) $C_{1}$, b) $C_{2}$, and c) the integrated cathode plume current, recreated from [45]. 


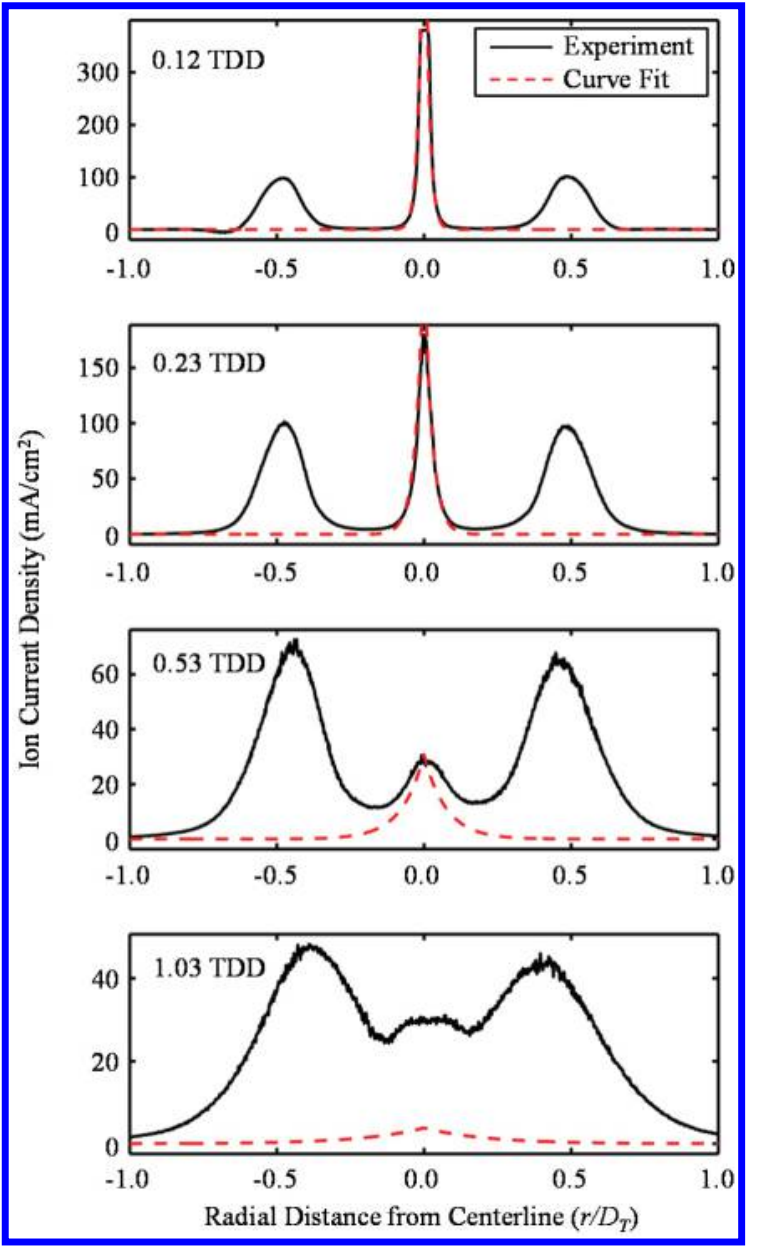

Fig. B3 Comparison of experimental data to curve-fitting results of the cathode plume, recreated from [45].

misalignment between the measured ion current density and the cathode plume, as shown in Fig. B1b on the thruster centerline at less than 0.2 TDD. Removal of the cathode plume is advised, even if the method generates spurious spikes or does not completely remove the cathode plume structure, possibly due to a low signal-to-noise ratio. The cathode plume remnants typically contribute less than a few percent to the integrated ion beam current due to the small integration element area. The effects may be included in error analysis. Overall, the cathode plume removal process is expected to improve analyses of beam divergence despite limitations of the correction. Additional details and recommendations are found in [45].

\section{References}

[1] Whited, J. B., "Summary of Electrical Propulsion Diagnostic Instrumentation," Arnold Engineering Development Center Rept. AEDC-TDR-63-242, Arnold AFB, TN, Jan. 1964.

[2] Hilton, H. H., and Stevens, J. R., "Faraday Cup Detectors," The Aerospace Corporation Rept. TR-0172(2260-20)-3, El Segundo, CA, June 1971.

[3] Pearlman, J. S., "Faraday Cups for Laser Plasmas," Review of Scientific Instruments, Vol. 48, No. 8, 1977, pp. 1064-1067. doi:10.1063/1.1135184

[4] Gerdin, G., Stygar, W., and Venneri, F., "Faraday Cup Analysis of Ion Beams Produced by a Dense Plasma Focus," Journal of Applied Physics, Vol. 52, No. 5, 1981, pp. 3269-3275. doi:10.1063/1.329145

[5] Jahn, R. G., Physics of Electric Propulsion, 1st ed., McGraw-Hill, New York, 1968, pp. 2-25.

[6] Goebel, D., and Katz, I., Fundamentals of Electric Propulsion: Ion and Hall Thrusters, Wiley, Hoboken, NJ, 2008, pp. 1-89.

- [7] Domonkos, M. T., Gallimore, A. D., Marrese, C. M., and Haas, J. M., "VeryNear-Field Plume Investigation of the Anode Layer Thruster," Journal of
Propulsion and Power, Vol. 16, No. 1, Jan.-Feb. 2000, pp. 91-98. doi: $10.2514 / 2.5536$

[8] Hofer, R. R., "Development and Characterization of High-Efficiency, High-Specific Impulse Xenon Hall Thrusters," Ph.D. Dissertation, Univ. of Michigan, Ann Arbor, MI, 2004.

[9] Liang, R., "The Combination of Two Concentric Discharge Channels into a Nested Hall-Effect Thruster," Ph.D. Dissertation, Univ. of Michigan, Ann Arbor, MI, 2013.

[10] Sekerak, M. J., Longmier, B. W., Gallimore, A. D., Brown, D. L., Hofer, R. R., and Polk, J. E., "Azimuthal Spoke Propagation in Hall Effect Thrusters," IEEE Transactions on Plasma Science, Vol. 43, No. 1, 2015, pp. 72-85. doi:10.1109/TPS.2014.2355223

[11] Lobbia, R. B., "A Time-Resolved Investigation of the Hall Thruster Breathing Mode," Ph.D. Dissertation, Univ. of Michigan, Ann Arbor, MI, 2010.

[12] Szabo, J., and Robin, M., "Plasma Species Measurements in the Plume of an Iodine Fueled Hall Thruster," Journal of Propulsion and Power, Vol. 30, No. 5, 2014, pp. 1357-1367. doi:10.2514/1.B35075

[13] Szabo, J., Robin, M., Paintal, S., Pote, B., and Hruby, V., "High Density Hall Thruster Propellant Investigations," AIAA Paper 2012-3853, July 2012.

[14] Szabo, J., Robin, M., Paintal, S., Pote, B., Hruby, V., and Freeman, C., "Iodine Plasma Propulsion Test Result sat 1-10 kW," IEEE Transactions on Plasma Science, Vol. 43, No. 1, 2015, pp. 141-148. doi:10.1109/TPS.2014.2367417

[15] Kerslake, W., and Ignaczak, L., "Development and Flight History of SERT II Spacecraft," AIAA Paper 1992-3516, July 1992.

[16] Brophy, J. R., Pless, L. C., and Garner, C. E., "Ion Engine Endurance Testing at High Background Pressures," AIAA Paper 1992-3205, July 1992.

[17] Randolph, T., Kim, V., Kaufman, H., Kozubsky, K., Zhurin, V., and Day, M., "Facility Effects on Stationary Plasma Thruster Testing," International Electric Propulsion Conference, Electric Rocket Propulsion Soc. Paper 1993-093, Sept. 1993, http://erps.spacegrant.org/.

[18] Brown, D. L., and Gallimore, A. D., "Evaluation of Facility Effects on Ion Migration in a Hall Thruster Plume," Journal of Propulsion and Power, Vol. 27, No. 3, 2011, pp. 573-585. doi:10.2514/1.B34068

[19] Walker, M. L. R., Victor, A. L., Hofer, R. R., and Gallimore, A. D., "Effect of Backpressure on Ion Current Density Measurements in Hall Thruster Plumes," Journal of Propulsion and Power, Vol. 21, No. 3, 2005, pp. 408-415. doi: $10.2514 / 1.7713$

[20] Walker, J., Frieman, J., Walker, M. L. R., and Khayms, V., "Hall Effect Thruster Electrical Interaction with a Conductive Vacuum Chamber," AIAA Paper 2014-3711, July 2014.

[21] Pollard, J. E., Diamant, K. D., Crofton, M. W., Patterson, M. J., and Soulas, G. C., "Spatially-Resolved Beam Current and Charge-State Distributions for the NEXT Ion Engine," AIAA Paper 2010-6779, July 2010.

[22] Mikellides, I. G., Katz, I., Kuharski, R. A., and Mandell, M. J., "Elastic Scattering of Ions in Electrostatic Thruster Plumes," Journal of Propulsion and Power, Vol. 21, No. 1, 2005, pp. 111-118. doi: $10.2514 / 1.5046$

[23] Soulas, G. C., "Modeling Neutral Densities Downstream of a Gridded Ion Thruster," AIAA Paper 2010-6699, July 2010.

[24] Boyd, I. D., and Yim, J. T., "Modeling of the Near-Field Plume of a Hall Thruster," Journal of Applied Physics, Vol. 95, No. 9, 2004, pp. 4575-4584. doi:10.1063/1.1688444

[25] Miller, J. S., Pullins, S. H., Levandier, D. J., Chiu, Y. H., and Dressler, R. A., "Xenon Charge Exchange Cross Sections for Electrostatic Thruster Models," Journal of Applied Physics, Vol. 91, No. 3, 2002, pp. 984-991. doi: $10.1063 / 1.1426246$

[26] Oh, D. O., "Computational Modeling of Expanding Plasma Plumes in Space Using a PIC-DSMC Algorithm," Ph.D. Dissertation, Massachusetts Inst. of Technology, Cambridge, MA, 1997.

[27] Wang, J., Brinza, D. E., Young, D. T., Nordholt, J. E., Polk, J. E., Henry, M. D., Goldstein, R., Hanley, J. J., Lawrence, D. J., and Shappirio, M., "Deep Space One Investigations of Ion Propulsion Plasma Environment," Journal of Spacecraft and Rockets, Vol. 37, No. 5, 2000 , pp. $545-555$. doi: $10.2514 / 2.3608$

[28] Davis, V. A., Mandell, M. J., Brinza, D. E., Henry, M. D., Wang, J. J., and Young, D. T., "Ion Engine Generated Charge Exchange Environment: Comparison Between NSTAR Flight Data and Numerical Simulations," AIAA Paper 2001-0970, Jan. 2001. 
[29] Oh, D. O., "Computational Modeling of Expanding Plasma Plumes in Space Using a PIC-DSMC Algorithm," Ph.D. Dissertation, Massachusetts Inst. of Technology, Cambridge, MA, 1997.

[30] Byers, D. C., "Angular Distribution of Kaufman Ion Thruster Beams," NASA TN D-5844, June 1970.

[31] Rovey, J. L., Walker, M. L. R., Gallimore, A. D., and Peterson, P. Y., "Magnetically Filtered Faraday Probe for Measuring the Ion Current Density Profile of a Hall Thruster," Review of Scientific Instruments, Vol. 77, No. 1, 2006, Paper 013503.

doi:10.1063/1.2149006

[32] Hofer, R. R., Walker, M. L. R., and Gallimore, A. D., "A Comparison of Nude and Collimated Faraday Probes for Use with Hall Thrusters," International Electric Propulsion Conference, Electric Rocket Propulsion Soc. Paper 2001-020, Oct. 2001, http://erps.spacegrant.org/.

[33] Boyd, I. D., and Dressler, R. A., "Far-Field Modeling of the Plasma Plume of a Hall Thruster," Journal of Applied Physics, Vol. 92, No. 4, 2002, pp. 1764-1774. doi:10.1063/1.1492014

[34] Mikellides, I. G., Jongeward, G. A., Katz, I., and Manzella, D. H., "Plume Modeling of Stationary Plasma Thrusters and Interactions with the Express-A Spacecraft," Journal of Spacecraft and Rockets, Vol. 39, No. 6, 2002, pp. 894-903. doi: $10.2514 / 2.3896$

[35] Passaro, A., Vicini, A., Nania, F., and Biagioni, L., "Numerical Rebuilding of SMART-1 Hall Effect Thruster Plasma Plume," Journal of Propulsion and Power, Vol. 26, No. 1, 2010, pp. 149-158. doi: $10.2514 / 1.36821$

[36] Mikellides, I. G., Kuharski, R. A., Mandell, M. J., and Gardner, B. M., "Assessment of Spacecraft Systems Integration Using the Electric Propulsion Interactions Code (EPIC)," AIAA Paper 2002-3667, July 2002.

[37] Wang, J., Brinza, D., Goldstein, R., Polk, J., Henry, M., Young, D. T., Hanley, J. J., Nordholt, J., Lawrence, D., and Shappirio, M., "Deep Space One Investigations of Ion Propulsion Plasma Interactions: Overview and Initial Results," AIAA Paper 1999-2971, June 1999.

[38] Pollard, J. E., Diamant, K. D., Khayms, V., Werthman, L., King, D. Q., and de Grys, K. H., "Ion Flux, Energy, and Charge-State Measurements for the BPT-4000 Hall Thruster," AIAA Paper 2001-3351, July 2001.

[39] Azziz, Y., Martinez-Sanchez, M., and Szabo, J. J., "Determination of In-Orbit Plume Characteristics from Laboratory Measurements," AIAA Paper 2006-4484, July 2006.

[40] Beyer, W. H., CRC Standard Mathematical Tables, 28th ed., CRC Press, Boca Raton, FL, 1987, pp. 242-245.

[41] Hofer, R. R., and Gallimore, A. D., "Recent Results from Internal and Very-Near-Field Plasma Diagnostics of a High Specific Impulse Hall Thruster," International Electric Propulsion Conference, Electric Rocket Propulsion Soc. Paper 2003-037, March 2003, http://erps. spacegrant.org/.

[42] Manzella, D., Jankovsky, R., Elliot, F., Mikellides, I., Jongeward, G., and Allen, D., "Hall Thruster Plume Measurements On-Board the Russian Express Satellites," International Electric Propulsion Conference, Electric Rocket Propulsion Soc. Paper 2001-044, Oct. 2001, http://erps.spacegrant.org/.

- [43] Haas, J. M., Gallimore, A. D., McFall, K., and Spanjers, G., "Development of a High-Speed, Reciprocating Electrostatic Probe System for Hall Thruster Interrogation," Review of Scientific Instruments, Vol. 71, No. 11, 2000, pp. 4131-4138. doi:10.1063/1.1318921

[44] Reid, B. M., and Gallimore, A. D., "Near-Field Ion Current Density Measurements of a 6-kW Hall Thruster," International Electric Propulsion Conference, Electric Rocket Propulsion Soc. Paper 2009124, Sept. 2009, http://erps.spacegrant.org/.

[45] Huang, W., Shastry, R., Herman, D. A., Soulas, G. C., and Kamhawi, H., "A New Method for Analyzing Near-Field Faraday Probe Data in Hall Thrusters," AIAA Paper 2013-4118, July 2013.

[46] Horowitz, P., and Hill, W., The Art of Electronics, 3rd ed., Cambridge Univ. Press, New York, 2015, p. 474.

[47] Yalin, A. P., Williams, J. D., Surla, V., and Zoerb, K. A., "Differential Sputter Yield Profiles of Molybdenum Due to Bombardment by Low Energy Xenon Ions at Normal and Oblique Incidence," Journal of Physics D: Applied Physics, Vol. 40, No. 10, 2007, pp. 3194-3202. doi:10.1088/0022-3727/40/10/025

[48] Yalin, A. P., Rubin, B., Domingue, S. R., Glueckert, Z., and Williams, J. D., "Differential Sputter Yields of Boron Nitride, Quartz, and Kapton Due to Low Energy Xe+ Bombardment," AIAA Paper 2007-5314, July 2007.

[49] Zoerb, K. A., Williams, J. D., Williams, D. D., and Yalin, A. P., "Differential Sputter Yields of Refractory Metals by Xenon, Krypton, and Argon Ion Bombardment at Normal and Oblique Incidences," International Electric Propulsion Conference, Electric Rocket Propulsion Soc. Paper 2005-293, Oct. 2005, http://erps.spacegrant. org/.

[50] Tondu, T., Chardon, J., and Zurbach, S., "Sputtering Yield of Potential Ceramics for Hall Effect Thruster Discharge Channel," International Electric Propulsion Conference, Electric Rocket Propulsion Soc. Paper 2011-106, Sept. 2011, http://erps.spacegrant.org/.

[51] Thomas, T. L., and Battle, E. L., "Effects of Contamination on Langmuir Probe Measurements in Glow Discharge Plasmas," Journal of Applied Physics, Vol. 41, No. 8, 1970, pp. 3428-3432. doi:10.1063/1.1659435

[52] Szuszczewicz, E. P., and Holmes, J. C., "Surface Contamination of Active Electrodes in Plasmas: Distortion of Conventional Langmuir Probe Measurements," Journal of Applied Physics, Vol. 46, No. 12, 1975, pp. 5134-5139. doi: $10.1063 / 1.321572$

[53] Mikellides, I. G., Katz, I., Hofer, R. R., and Goebel, D. M., "Magnetic Shielding of Walls from the Unmagnetized Ion Beam in a Hall Thruster," Applied Physics Letters, Vol. 102, No. 2, 2013, Paper 023509. doi:10.1063/1.4776192

[54] Huang, W., Shastry, R., Herman, D. A., Soulas, G. C., and Kamhawi, H., "Ion Current Density Study of the NASA-300M and NASA457Mv2 Hall Thrusters," AIAA Paper 2012-3870, July 2012.

[55] Williams, J. D., Johnson, M. L., and Williams, D. D., "Differential Sputtering Behavior of Pyrolytic Graphite and Carbon-Carbon Composite Under Xenon Bombardment," AIAA Paper 2004-3788, July 2004.

[56] Chapman, B., Glow Discharge Processes: Sputtering and Plasma Etching, Wiley, New York, 1980, p. 45.

[57] Dankanich, J. W., Walker, M. L. R., Swiatek, M. W., and Yim, J. T., "Recommended Practice for Pressure Measurement and Calculation of Effective Pumping Speed in Electric Propulsion Testing," Journal of Propulsion and Power (accepted for publication).

[58] Chen, F. F., "Electric Probes," Plasma Diagnostic Techniques, edited by Huddlestone, R. H., and Leonard, S. L., Academic Press, New York, 1965, pp. 113-200.

[59] Malik, S. M., Fetherston, R. P., Sridharan, K., and Conrad, J. R., "Sheath Dynamics and Dose Analysis for Planar Targets in Plasma Source Ion Implantation," Plasma Sources Science and Technology, Vol. 2, No. 2, 1993, 1993, pp. 81-85. doi:10.1088/0963-0252/2/2/002

[60] Lobbia, R. B., and Beal, B. E., "Recommended Practice for Use of Langmuir Probes in Electric Propulsion Testing," Journal of Propulsion and Power (accepted for publication).

[61] Foster, J. E., Soulas, G. C., and Patterson, M. J., "Plume and Discharge Plasma Measurements of an NSTAR-type Ion Thruster," AIAA Paper 2000-3812, July 2000.

[62] Incropera, F. P., and DeWitt, D. P., Fundamentals of Heat and Mass Transfer, 5th ed., Wiley, Hoboken, NJ, 2002, p. 691.

[63] Dushman, S., "Electron Emission from Metals as a Function of Temperature," Physical Review, Vol. 21, No. 6, 1923, pp. 623-636. doi:10.1103/PhysRev.21.623

[64] Swanson, L. W., and Schwind, G. A., "Review of ZrO/W Schottky Cathode," Handbook of Charged Particle Optics, 2nd ed., CRC Press, Boca Raton, FL, 2009, pp. 5-6.

[65] Lassner, E., and Schubert, W. D., Tungsten: Properties, Chemistry, Technology of the Element, Alloys, and Chemical Compounds, Kluwer Academic/Plenum, New York, 1999, pp. 40-42.

[66] Nottingham, W. B., "Thermionic Emission from Tungsten and Thoriated Tungsten Filaments," Physical Review, Vol. 49, No. 1, 1936, pp. 78-97. doi:10.1103/PhysRev.49.78

[67] Murphy, E. L., and Good, R. H., Jr., "Thermionic Emission, Field Emission, and the Transition Region," Physical Review, Vol. 102, No. 6, 1956, pp. 1464-1473. doi:10.1103/PhysRev.102.1464

[68] Nakles, M. R., and Hargus, W. A., Jr., "Background Pressure Effects on Ion Velocity Distribution Within a Medium-Power Hall Thruster," Journal of Propulsion and Power, Vol. 27, No. 4, 2011, pp. 737-743. doi: $10.2514 / 1.48027$

[69] Foster, J. E., Williams, G. J., and Patterson, M. J., "Characterization of an Ion Thruster Neutralizer," Journal of Propulsion and Power, Vol. 23, No. 4, 2007, pp. 828-835. doi: $10.2514 / 1.22591$

[70] Brown, D. L., "Investigation of Low Discharge Voltage Hall Thruster Characteristics and Evaluation of Loss Mechanisms," Ph.D. Dissertation, Univ. of Michigan, Ann Arbor, MI, 2009.

[71] Brown, D. L., and Gallimore, A. D., "Investigation of Low Discharge Voltage Hall Thruster Operating Modes and Ionization Processes," 
International Electric Propulsion Conference, Electric Rocket Propulsion Soc. Paper 2009-074, Sept. 2009, http://erps.spacegrant.org/.

[72] Sekerak, M. J., "Plasma Oscillations and Operational Modes in Hall Effect Thrusters," Ph.D. Dissertation, Univ. of Michigan, Ann Arbor, MI, 2014.

[73] Brown, D. L., Lobbia, R. B., and Blakely, J. M., "Low-Voltage Hall Thruster Mode Transitions," AIAA Paper 2014-3510, July 2014.

[74] Polk, J. E., Kakuda, R. Y., Anderson, J. R., and Brophy, J. R., "Demonstration of the NSTAR Ion Propulsion System on the Deep Space One Mission," International Electric Propulsion Conference, Electric Rocket Propulsion Soc. Paper 2001-075, Jan. 2001, http://erps spacegrant.org/.

[75] Brophy, J. R., Garner, C. E., and Mikes, S., "Dawn Ion Propulsion System: Initial Checkout After Launch," Journal of Propulsion and Power, Vol. 25, No. 6, 2009, pp. 1189-1202. doi: $10.2514 / 1.40480$

[76] Brophy, J. R., Pless, L. C., and Garner, C. E., "Ion Engine Endurance Testing at High Background Pressures," AIAA Paper 1992-3205, July 1992.

- [77] Brown, D. L., and Gallimore, A. D., "Evaluation of Ion Collection Area in Faraday Probes," Review of Scientific Instruments, Vol. 81, No. 6, 2010, Paper 063504.

doi: $10.1063 / 1.3449541$

[78] Hagstrum, H. D., "Auger Ejection of Electrons from Tungsten by Noble Gas Ions," Physical Review, Vol. 96, No. 2, 1954, pp. 325-335. doi:10.1103/PhysRev.96.325

[79] Hagstrum, H. D., "Auger Ejection of Electrons from Molybdenum by Noble Gas Ions," Physical Review, Vol. 104, No. 3, 1956, pp. $672-683$. doi:10.1103/PhysRev.104.672

-[80] Hagstrum, H. D., "Auger Ejection of Electrons from Tungsten by Noble Gas Ions," Physical Review, Vol. 104, No. 2, 1956, pp. 317-318. doi:10.1103/PhysRev.104.317

[81] Shastry, R., Hofer, R. R., Reid, B. M., and Gallimore, A. D., "Method for Analyzing ExB Probe Spectra from Hall Thruster Plumes," Review of Scientific Instruments, Vol. 80, No. 6, 2009, Paper 063502. doi: $10.1063 / 1.3152218$

[82] Reid, B. M., Shastry, R., Gallimore, A. D., and Hofer, R. R., "Angularly Resolved ExB Probe Spectra in the Plume of a 6-kW Hall Thruster," AIAA Paper 2008-5287, July 2008.

[83] Draper, N. D., and Smith, H., "Chapter 1 Fitting a Straight Line by Least Squares," Applied Regression Analysis, 3rd ed., Wiley, New York, 1998, pp. 15-46.

[84] de Grys, K. H., Tilley, D. L., and Aadland, R. S., "BPT Hall Thruster Plume Characteristics," AIAA Paper 1999-2283, June 1999.

[85] Reid, B. M., "The Influence of Neutral Flow Rate in the Operation of Hall Thrusters," Ph.D. Dissertation, Univ. of Michigan, Ann Arbor, MI, 2009.

[86] Azziz, Y., "Experimental and Theoretical Characterization of a Hall Thruster Plume," Ph.D. Dissertation, Massachusetts Inst. of Technology, Cambridge, MA, 2007.

[87] Mundy, D. H., "Factors Affecting the Beam Divergence of a T5 Ion Engine," International Electric Propulsion Conference, Electric Rocket Propulsion Soc. Paper 1997-095, Oct. 1997, http://erps. spacegrant.org/.

[88] Brown, D. L., Larson, C. W., Beal, B. E., and Gallimore, A. D., "Methodology and Historical Perspective of a Hall Thruster Efficiency Architecture," Journal of Propulsion and Power, Vol. 25, No. 6, 2009, pp. 1163-1177. doi:10.2514/1.38092

[89] Boyd, I. D., and Crofton, M. W., "Numerical Momentum Tracking for a Hall Thruster Plume," AIAA Paper 2014-3428, July 2014.

[90] Kim, S., Foster, J. E., and Gallimore, A. D., "Very-Near-Field Plume Study of a 1.35 kW SPT-100," AIAA Paper 1996-2972, July 1996.

[91] Myers, R. M., Pencil, E. J., Rawlin, V. K., Kussmaul, M., and Oden, K., "NSTAR Ion Thruster Plume Impacts Assessments," AIAA Paper 1995-2825, July 1995.

[92] Soulas, G. C., Foster, J. E., and Patterson, M. J., "Performance of Titanium Optics on a NASA $30 \mathrm{~cm}$ Ion Thruster," AIAA Paper 20003814, July 2000.

[93] Soulas, G. C., Haag, T. W., and Patterson, M. J., "Performance Evaluation of $40 \mathrm{~cm}$ Ion Optics for the NEXT Ion Engine," AIAA Paper 2002-3834, July 2002.

[94] Polk, J. E., Kakuda, R. Y., Anderson, J. R., Brophy, J. R., Rawlin, V. K., Patterson, M. J., Sovey, J., and Hamley, J., "Validation of the NSTAR Ion Propulsion System on Deep Space One Mission: Overview and Initial Results," AIAA Paper 1999-2274, June 1999.

[95] Foster, J. E., Soulas, G. C., and Patterson, M. J., "Plume and Discharge Plasma Measurements of an NSTAR-Type Ion Thruster," AIAA Paper 2000-3812, July 2000.
[96] Reid, B. M., Shastry, R., Gallimore, A. D., and Hofer, R. R., "Angularly-Resolved ExB Probe Spectra in the Plume of a 6-kW Hall Thruster," AIAA Paper 2008-5287, July 2008.

[97] Huang, W., Kamhawi, H., and Haag, T., "Effect of Background Pressure on the Performance and Plume of the HiVHAc Hall Thruster," International Electric Propulsion Conference, Electric Rocket Propulsion Soc. Paper 2013-058, Oct. 2013, http://erps.spacegrant. org/.

[98] Byers, D., and Dankanich, J. W., "A Review of Facility Effects on Hall Effect Thrusters," International Electric Propulsion Conference, Electric Rocket Propulsion Soc. Paper 2009-076, Sept. 2009, http:// erps.spacegrant.org/.

[99] Hofer, R. R., Peterson, P. Y., and Gallimore, A. D., "Characterizing Vacuum Facility Backpressure Effects on the Performance of a Hall Thruster," International Electric Propulsion Conference, Electric Rocket Propulsion Soc. Paper 2001-045, Oct. 2001, http://erps. spacegrant.org/.

[100] Nakles, M. R., Brieda, L., Reed, G. D., Hargus, W. A., Jr., and Spicer, R. L., "Experimental and Numerical Examination of the BHT-200 Hall Thruster Plume," AIAA Paper 2007-5305, July 2007.

[101] "Evaluation of Measurement Data-Guide to the Expression of Uncertainty in Measurement," 1st ed., Joint Committee for Guides in Metrology Rept. JCGM 100:2008, 2008, pp. 1-51, http://www.bipm. org/en/publications/guides/ [retrieved Oct. 2015].

[102] "International Vocabulary of Metrology-Basic and General Concepts and Associated Terms," 3rd ed., Joint Committee for Guides in Metrology Rept. JCGM 200:2008, 2008, pp. 1-34, http://www.bipm. org/en/publications/guides/ [retrieved Oct. 2015].

[103] Farnell, Casey C., Farnell, Cody C., Farnell, S. C., and Williams, J. D., "Recommended Practice for Use of Electrostatic Analyzers in Electric Propulsion Testing," Journal of Propulsion and Power (accepted for publication).

[104] Yamamura, Y., "Theory of Sputtering and Comparison to Experimental Data," Nuclear Instruments and Methods in Physics Research, Vol. 194, Nos. 1-3, 1982, pp. 515-522. doi:10.1016/0029-554X(82)90575-4

[105] Boyd, I. D., and Falk, M. L., "A Review of Spacecraft Material Sputtering By Hall Thruster Plumes," AIAA Paper 2001-3353, July 2001.

[106] Reid, B. M., and Gallimore, A. D., "Plasma Potential Measurements in the Discharge Channel of a 6-kW Hall Thruster," AIAA Paper 20085185, July 2008.

[107] Dunaevsky, A., Raitses, Y., and Fisch, N. J., "Secondary Electron Emission from Dielectric Materials of a Hall Thruster with Segmented Electrodes," Physics of Plasmas, Vol. 10, No. 6, 2003, pp. 2574-2577. doi:10.1063/1.1568344

[108] "COMBAT® Boron Nitride Solids," Saint-Gobain Ceramic Materials, Product Datasheet, SGBN-Combat Solids-DS1-0214-R03, Amherst, NY, 2011, http://www.bn.saint-gobain.com/uploadedFiles/SGbn/ Documents/Solids/Combat-Solids-DS1.pdf, [retrieved Oct. 2015].

[109] "Boron Nitride XP Grade Material Properties," Accuratus Ceramic Corp., Produce Datasheet, Phillipsburg, NJ, http://accuratus.com/pdf/ BNXPprops.pdf [retrieved Oct. 2015].

[110] Hussey, R. J., and Wilson, J., Advanced Technical Ceramics Directory and Databook, 1st ed., Chapman and Hall, New York, 1998, pp. 106-109. doi:10.1007/978-1-4419-8662-7

[111] "MACOR ${ }^{\circledR}$ Machinable Glass Ceramic for Industrial Applications," Corning, Inc., Avon, France, http://www.corning.com/media/worldwide/ csm/documents/71759a443535431395eb34ebead091cb.pdf [retrieved Oct. 2015].

[112] Tartz, M., Heyn, T., Bundesmann, C., and Neumann, H., "Measuring Sputter Yields of Ceramic Materials," International Electric Propulsion Conference, Electric Rocket Propulsion Soc. Paper 2009-240, Sept. 2009, http://erps.spacegrant.org/.

[113] Hofer, R. R., and Gallimore, A. D., "Recent Results from Internal and Very-Near-Field Plasma Diagnostics of a High Specific Impulse Hall Thruster," International Electric Propulsion Conference, Electric Rocket Propulsion Soc. Paper 2003-037, March 2003, http://erps. spacegrant.org/.

[114] Haas, J. M., Gallimore, A. D., McFall, K., and Spanjers, G., "Development of a High-Speed, Reciprocating Electrostatic Probe System for Hall Thruster Interrogation," Review of Scientific Instruments, Vol. 71, No. 11, 2000, pp. 4131-4138. doi:10.1063/1.1318921

[115] Ziemer, J. K., "Performance of Electrospray Thrusters," International Electric Propulsion Conference, Electric Rocket Propulsion Soc. Paper 2009-242, Sept. 2009, http://erps.spacegrant.org/. 
[116] Martinez-Sanchez, M., Fernandez de la Mora, J., Hruby, V., GameroCastaño, M., and Khayms, V., "Research on Colloid Thrusters," International Electric Propulsion Conference, Electric Rocket Propulsion Soc. Paper 1999-014, Oct. 1999, http://erps.spacegrant.org/.

[117] Demmons, N., Hruby, V., Spence, D., Roy, T., Ehrbar, E., Zwahlen, J., and Martin, R., "ST7-DRS Mission Colloid Thruster Development," AIAA Paper 2008-4823, July 2008.

[118] Wilbur, P. J., Jahn, R. G., and Curran, F. C., "Space Electric Propulsion Plasmas," IEEE Transactions on Plasma Science, Vol. 19, No. 6, 1991, pp. 1167-1179. doi:10.1109/27.125039

[119] Pugmire, T. K., Shaw, R., and Enos, G. R., "Applied Resistojet Technology," Journal of Spacecraft and Rockets, Vol. 8, No. 1, 1971, pp. 63-68. doi: $10.2514 / 3.30218$

[120] Tiliakos, N. T., Burton, R. L., and Krier, H., "Arcjet Anode Plasma Measurements Using Electrostatic Probes," Journal of Propulsion and Power, Vol. 14, No. 4, 1998, pp. 560-567. doi: $10.2514 / 2.5315$

121] Gallimore, A. D., Kim, S., Foster, J. E., King, L. B., and Gulczinski, F S., "Near- and Far-Field Plume Studies of a One-Kilowatt Arcjet," Journal of Propulsion and Power, Vol. 12, No. 1, 1996, pp. 105-111. doi: $10.2514 / 3.23997$

[122] Bufton, S. A., and Burton, R. L., "Velocity and Temperature Measurements in a Low-Power Hydrazine Arcjet," Journal of Propulsion and Power, Vol. 13, No. 6, 1997, pp. 768-774. doi: $10.2514 / 2.5231$

[123] Crofton, M. W., Moore, T. A., Boyd, I. D., Masuda, I., and Gotoh, Y., "Near-Field Measurement and Modeling Results for Flight-Type Arcjet: NH Molecule," Journal of Spacecraft and Rockets, Vol. 38, No. 1, 2001, pp. 79-86 doi: $10.2514 / 2.3657$

[124] Crofton, M. W., Moore, T. A., Boyd, I. D., Masuda, I., and Gotoh, Y., "Near-Field Measurement and Modeling Results for Flight-Type Arcjet: Hydrogen Atom," Journal of Spacecraft and Rockets, Vol. 38, No. 3, 2001, pp. 417-425. doi: $10.2514 / 2.3700$

[125] Butler, G. W., and Cassady, R. J., "Directions for Arcjet Technology Development," Journal of Propulsion and Power, Vol. 12, No. 6, 1996 , pp. $1026-1034$.

doi: $10.2514 / 3.24140$

[126] Carney, L. M., and Sankovic, J. M., "The Effects of Arcjet Thruster Operating Conditions and Constrictor Geometry on the Plasma Plume," AIAA Paper 1989-2723, July 1989.

[127] Lau, M., Manna, S., Herdrich, G., Schonherr, T., and Komurasaki, K., "Experimental Investigation of the Current Density in the Discharge Plasma of ADD SIMP-LEX," AIAA Paper 2012-4275, July 2012.

[128] Myers, R. M., Arrington, L. A., Pencil, E. J., Carter, J., Heminger, J., and Gatsonis, N., "Pulsed Plasma Thruster Contamination," AIAA Paper 1996-2729, July 1996.

[129] Myers, R. M., "Plume Characteristics of MPD Thrusters: A Preliminary Investigation,” AIAA Paper 1989-2832, July 1989.

[130] Sankaran, K., Choueiri, E. Y., and Jardin, S. C., "Comparison of Simulated Magnetoplasmadynamic Thruster Flowfields to Experimental Measurements," Journal of Propulsion and Power, Vol. 21, No. 1, 2005, pp. 129-138 doi: $10.2514 / 1.5340$

[131] Eckman, R., Byrne, L., Gatsonis, N. A., and Pencil, E. J., "Triple Langmuir Probe Measurements in the Plume of a Pulsed Plasma Thruster," Journal of Propulsion and Power, Vol. 17, No. 4, 2001, pp. 762-771. doi: $10.2514 / 2.5831$

[132] Kodys, A. D., and Choueiri, E. Y., "A Critical Review of the State-ofthe-Art in the Performance of Applied-Field Magnetoplasmadynamic Thrusters," AIAA Paper 2005-4247, July 2005.

[133] Krulle, G., Auweter-Kurtz, M., and Sasoh, A., "Technology and Application Aspects of Applied Field Magnetoplasmadynamic Propulsion," Journal of Propulsion and Power, Vol. 14, No. 5, 2005, pp. 754-763. doi: $10.2514 / 2.5338$

[134] Sovie, R. J., and Connolly, D. J., "Effects of Backgrounds Pressure on Magnetoplasmadynamic Thruster Operation," Journal of Spacecraft and Rockets, Vol. 7, No. 3, 1970, pp. 255-258. doi: $10.2514 / 3.29916$

J. Blandino Associate Editor 
This article has been cited by:

1. Thomas Trottenberg, Alexander Spethmann, Holger Kersten. 2018. An interferometric force probe for beam diagnostics and the study of sputtering. EPJ Techniques and Instrumentation 5:1. . [Crossref]

2. Stephane Mazouffre, Lou Grimaud. 2018. Characteristics and Performances of a 100-W Hall Thruster for Microspacecraft. IEEE Transactions on Plasma Science 46:2, 330-337. [Crossref]

3. Jason D. Frieman, Nathan P. Brown, Connie Y. Liu, Thomas M. Liu, Mitchell L. R. Walker, Vadim Khayms, David Q. King. 2018. Electrical Facility Effects on Faraday Probe Measurements. Journal of Propulsion and Power 34:1, 267-269. [Citation] [Full Text] [PDF] [PDF Plus] 